\title{
Optique géométrique
}

\author{
N. Vansteenkiste-Westbrook \\ Institut d'Optique, B.P. 147, 91403 Orsay cedex, France
}

\begin{abstract}
Résumé : L'objectif de ce cours est de rappeler les bases de l'optique géométrique, principalement dans le domaine de l'approximation de Gauss. Le point de départ est le principe de Fermat ou les lois de Descartes pour la propagation des rayons. Après avoir posé le problème du stigmatisme pour la formation des images et évoqué le cas du stigmatisme approché (relation d'aplanétisme), le formalisme général dc l'approximation linéaire est démontré et appliqué à l'étude des systèmes simples ou associés. Ceci est ensuite appliqué à l'étude des instruments, du point de vue dimension des images, champ en largeur et en profondeur, et photométrie.
\end{abstract}

\section{PROPAGATION DES RAYONS}

\subsection{Rappels des principes généraux}

Plusieurs approches sont possibles avec comme point de départ le principe de Fermat, ou le principe d'Huyghens, ou la détermination expérimentale des lois de la réfraction et de la réflexion ; elles conduisent toutes à un bon accord avec les observations expérimentales. Il s'agit donc suivant le problème posé de choisir l'approche la mieux adaptée : c'est le cas du principe de Fermat pour l'étude des milieux à gradient d'indice (milieux non homogènes) ou de la construction d'Huyghens pour la propagation des rayons dans des milieux biréfringents (anisotropes).

Nous présenterons dans cette partie le principe de Fermat et les lois de Descartes, ainsi que le théorème de Malus-Dupin, permettant de relier les trajectoires des rayons aux aspects ondulatoires. Nous nous limiterons parfois à des milieux homogènes et/ou isotropes, dont nous rappelons ici les caractéristiques :

*un milieu est homogène pour l'onde lumineuse si l'indice ne dépend pas du point considéré dans le milieu à l'échelle de la longueur d'onde ;

*un milieu est isotrope pour l'onde lumineuse si l'indice ne dépend pas de la direction de polarisation de la lumière.

Une fibre optique dont l'indice décroît avec la distance à l'axe (fibre à gradient d'indice) est un exemple de milieu inhomogène et isotrope. Un cristal parfait dont la maille élémentaire n'a pas la symétrie de révolution (milieu biréfringent par exemple le quartz) est un milieu anisotrope mais homogène. 


\subsubsection{Principe de Fermat}

Le physicien français Pierre de Fermat a proposé en 1657 un principe général de propagation des rayons lumineux, que l'on peut écrire sous la forme suivante :

\section{La lưmière suit le trajet correspondant à un chemin optique stationnaire.}

Le chemin optique stationnaire correspond à un temps stationnaire : c'est en effet le chemin que parcourrait la lumière dans le même temps si elle se propageait dans le vide. Temps de parcours et chemin optique sont donc reliés par la vitesse de la lumière dans le vide $c$.

Ce principe de propagation a deux conséquence immédiates:

- la lumière se propage en ligne droite dans un milieu họogène ;

- le trajet suivi par la lumière ne dépend pas du sens de parcours, quel que soit le milieu de propagation (notons que ceci ne préjuge en rien de la répartition d'énergie dans les faisceaux aller et retour, qui peut ne pas être symétrique, en particulier lorsqu'on prend en compte les phénomènes de polarisation).

Le principe de Fermat est bien adapté à la détermination des trajectoires suivies par la lumière dans les milieux inhomogènes (voir partie 1.2).

On parlera souvent abusivement de chemin optique entre deux points $A$ et $B$ sans préciser pour quelle trajectoire, auquel cas on sous-entend le chemin effectivement suivi par la lumière (donc celui qui est stationnaire). Dans le cas particulier d'un milieu homogène et isotrope d'indice $\mathrm{n}$ uniforme, ce chemin optique sera tout simplement $L=n \overline{\mathrm{AB}}$. Dans le cas d'un milieu anisotrope, les rayons et les vecteurs d'onde ne sont plus colinéaires et le chemin optique doit être compté le long des vecteurs d'onde avec l'indice correspondant à la vitesse de propagation de l'onde.

\subsubsection{Lois de Snell-Descartes}

On peut montrer assez facilement que le principe de Fermat permet de retrouver les lois de la réfraction et de la réflexion à la traversée d'une surface séparant deux milieux homogènes. Nous nous contenterons ici de rappeler ces lois.

Loi de Snell-Descartes vectorielle relative à la réfraction : $n_{1} u_{1}-n_{2} u_{2}=\left(n_{1} \cos i_{1}-n_{2} \cos i_{2}\right) \mathbf{N}$

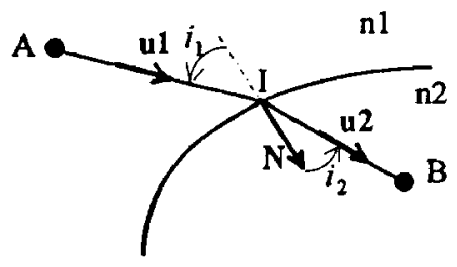

On peut l'exprimer de façon équivalente sous la forme des deux propriétés suivantes :

* le rayon réfracté est contenu dans le plan d'incidence, plan défini par le rayon incident et la normale au dioptre ;

* en se plaçant dans le plan d'incidence et en multipliant vectoriellement la loi précédente $\operatorname{par} \mathbf{N}$, on obtient la loi de la réfraction : 


$$
n_{1} \sin i_{1}=n_{2} \sin i_{2}
$$

Loi de Snell-Descartes vectorielle relative à la réflexion :

$$
\mathbf{u}_{1}-\mathbf{u}_{2}=2 \cos i_{1} \mathbf{N}
$$

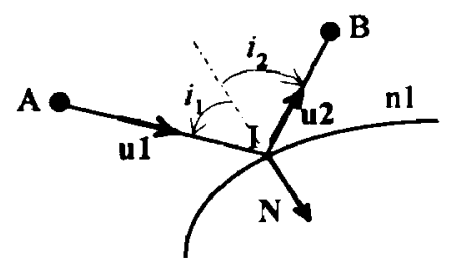

ou encore :

* le rayon réfléchi est contenu dans le plan d'incidence

$* i_{1}=-i_{2}$

On peut noter que les lois de la réflexion sont contenues dans les lois de la réfraction en prenant $n_{1}=n_{2}$ et la convention précisée sur la figure pour les vecteurs unitaires.

Quelques conséquences pratiques:

- on se rapproche de la normale lorsqu'on se réfracte dans un milieu plus réfringent (d'indice plus fort) et on s'en écarte lorsqu'on passe dans un milieu moins réfringent.

- le principe de retour inverse de la lumière nous permet de voir que par réfraction à travers une lame à faces planes et parallèles (ou plusieurs superposées), un rayon ressort toujours parallèle à lui-même.

- au passage vers un milieu moins réfringent (par exemple de l'eau d'indice 1.3 vers l'air d'indice 1), il existe un angle limite au delà duquel il n'y a plus réfraction. C'est le phénomène de réflexion totale qui se produit pour un angle d'incidence limite $i_{L}$ donné par :

$$
\sin i_{L}=\frac{n_{2}}{n_{1}}
$$

Cet angle vaut environ $42^{\circ}$ au passage verre/air $(n=1.5)$ et $49^{\circ}$ au passage eau/air.

\subsubsection{Lien rayons/surfaces d'onde : théorème de Malus-Dupin.}

L'optique géométrique est une approximation qui permet de caractériser de façon simplifiée la propagation de la lumière en termes de rayons lumineux. On peut grâce par exemple aux lois de Snell-Descartes, calculer la trajectoire d'un rayon quelconque à travers un système, et ce pour un grand nombre de rayons (principe du calcul optique par ordinateur). Il est alors intéressant de pouvoir relier ces rayons à l'aspect ondulatoire de la lumière, afin de pouvoir faire intervenir les phénomènes d'interférence ou de diffraction. Le théorème de Malus-Dupin montre comment relier les rayons aux surfaces d'onde dans le cas d'un milieu isotrope.

1.1.3. 1 Rappel sur les surfaces d'onde. Une surface d'onde est une surface équiphase du champ électromagnétique. Elle est obtenue à partir d'un point source en portant le même chemin optique le long de toutes les trajectoires. Pour un point source $\mathrm{S}$ à distance finie dans un milieu 
homogène et isotrope, les surfaces d'onde sont donc des surfaces de sphères centrées en $\mathrm{S}$. Si le point source est à l'infini, les surfaces d'onde deviennent des plans.

1.1.3.2 Théorème de Malus-Dupin. Dans un milieu isotrope, les surfaces d'onde sont orthogonales aux rayons lumineux. Cette propriété peut se montrer de façon générale à partir des équations de Maxwell : on montre alors que les surfaces équiphases sont orthogonales au vecteur d'onde, parallèle au vecteur de Poynting dans le cas d'un milieu isotrope. On peut également le démontrer en utilisant le principe de Fermat.

\subsection{Application du principe de Fermat aux milieux inhomogènes}

\subsection{Approche élémemaire dans le cas d'un gradient d'indice à une dimension}

Considérons un milieu inhomogène simple dont lindice $\mathrm{n}$ est indépendant de $\mathrm{x}$ et $\mathrm{y}$ et augmente linéairement avec $\mathbf{z}$. On peut essayer de deviner comment vont se propager les rayons dans un tel milieu en appliquant les lois de la réfraction sur des tranches infiniment fines de hauteur $\mathrm{dz}$.

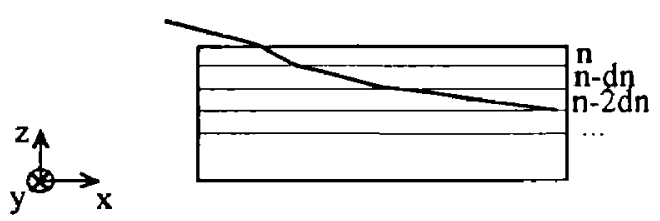

Au fur et à mesure que l'indice diminue, l'angle réfracté augmente et le rayon se courbe vers la zone d'indice plus fort. Afin d'avoir une approche un peu plus quantitative, nous allons revenir au principe de Fermat.

\subsubsection{Trajectoire d'un rayon lumineux dans un milieu inhomogène.}

Dans le cas général d'un milieu inhomogène, le principe de Fermat est le mieux adapté à l'étude de la propagation des rayons. Le calcul de l'équation générale de la trajectoire d'un rayon lumineux est effectué en différenciant le chemin optique et en annulant cette différentielle puisque le chemin doit être stationnaire. Nous nous contenterons ici d'en donner le résultat :

$$
\frac{d(n \mathbf{u})}{d s}=\operatorname{grad} n
$$

Cette équation définit la trajectoire d'un rayon lumineux dont u est le vecteur tangent, dans un milieu inhomogène caractérisé par le vecteur grad $n$ en tout point. On peut encore écrire cette équation en fonction de $\mathrm{R}$ rayon de courbure de la trajectoire, $\mathbf{u}$ et $\mathbf{N}$ vecteurs unitaires respectivement tangent et normal au rayon : 


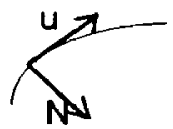

$\frac{d \mathbf{u}}{d s}=\frac{\mathbf{N}}{R}$ avec $R>0$ en prenant $\mathbf{N}$ dirigé vers le centre de courbure; d'où la nouvelle équation du rayon lumineux :

$$
\frac{d n}{d s} \mathbf{u}+\frac{n \mathbf{N}}{R}=\operatorname{grad} n
$$

En multipliant scalairement les deux membres de cette équation par $\mathbf{N}$, on obtient l'expression de la courbure de la trajectoire:

$$
\frac{1}{R}=\frac{1}{n} \operatorname{grad} n \cdot \mathbf{N}
$$

Comme $\mathrm{R}$ est positif, l'angle entre grad $n$ et $\mathbf{N}$ est toujours inférieur à $90^{\circ}$. La concavité de la trajectoire est donc toujours tournée dans le sens du vecteur grad $n$ (vers les zones de plus fort indice). C'est d'ailleurs bien ce șu'on avait trouvé avec l'approche élémentaire.

\subsubsection{Exemples d'application}

1.2.3.1 Mirages. Lorsque le sol est très chaud, il apparaît un gradient de température de l'air, plus chaud au voisinage du sol et diminuant lorsqu'on s'en éloigne. Or l'indice de l'air (qui vaut environ 1.0003) diminue quand la température augmente $\left(\Delta \mathrm{V} / \Delta \mathrm{T}=-10^{-6} /{ }^{\circ} \mathrm{C}\right)$. L'air au voisinage du sol est donc un milieu inhomogène dont le vecteur grad $n$ est vertical dirigé vers le haut. Les rayons provenant d'un objet s'incurvent et atteignent l'observateur en semblant s'être réfléchis sur une surface réfléchissante telle une nappe d'eau.

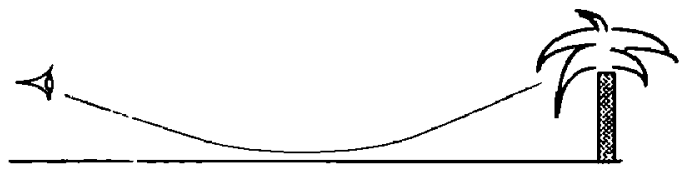

L'effet mirage est aussi utilisé à l'étude des caractéristiques thermiques de matériaux par voie optique en mesurant le déplacement d'un faisceau en fonction de l'échauffement.

1.2.3.2 Réfraction atmosphérique. L'atmosphère terrestre présente une variation continue de l'indice avec l'altitude liée à la diminution de sa densité : on passe d'environ 1.0003 au niveau du sol à 1 lorsqu'on atteint le vide. Les rayons issus d'une étoile se courbent à la traversée de l'atmosphère de telle sorte que l'angle sous lequel cette étoile est vue depuis la Terre est modifié (environ 1' d'arc) 


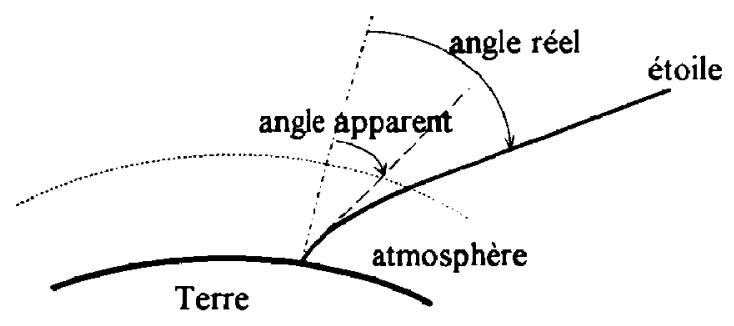

En particulier, le soleil peut être encore visible alors qu'il a dépassé la ligne théorique d'horizon. Si on ajoute à cela le fait que les rayons de courte longueur d'onde (bleu) sont plus déviés que ceux de plus grande longueur d'onde (rouge) car l'indice est plus fort aux courtes longueurs d'onde, et le fait que la sensibilité de l'oeil est maximale dans le jaune-vert, on obtient le phénomène du "rayon vert", visible au crépuscule juste après que le soleil ne se couche ou le matin juste avant qu'il se lève (à condition que les conditions météorologiques soient très bonnes, et de préférence en montagne).

1.2.3.3 Fibres optiques et lentilles à gradient d'indice. Pour les fibres optiques à saut d'indice, formées de deux milieux homogènes, le cœur d'indice plus fort et la gaine d'indice plus faible, la propagation des rayons peut s'expliquer en termes de réflexion totale à l'interface cour-gaine.

Le schéma suivant illustre le principe des fibres à gradient d'indice, en montrant l'allure du profil d'indice en coupe et la trajectoire des rayons :

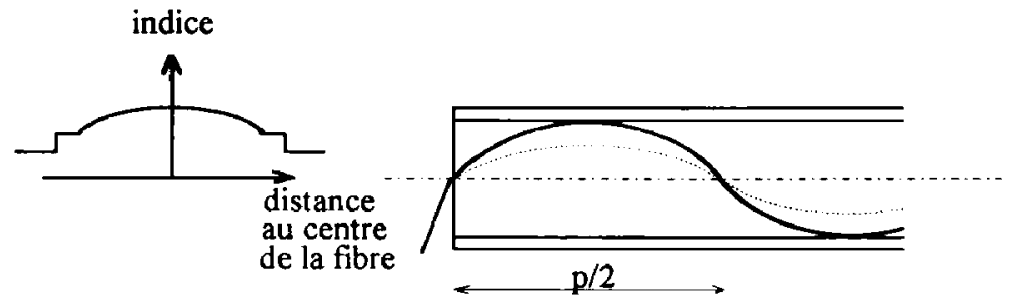

On peut montrer que pour un gradient d'indice bien choisi (du type $n=n_{0}-\alpha r^{2}$ ), toutes les trajectoires sont sinusoïdales de même période $\mathrm{p}$. Si on coupe une section d'une telle fibre d'une longueur inférieure à $\mathrm{p} / 2$, on obtient alors une lentille à gradient d'indice dont les caractéristiques dépendent de la longueur choisie.

\subsection{Application des lois de Descartes aux miroirs plans}

\subsubsection{Propriétés élémentaires}

Un miroir plan est une surface plane réfléchissant au moins partiellement la lumière. Le rayon réfléchi est contenu dans le plan d'incidence et il est symétrique du rayon incident par rapport à 
la normale. L'ensemble des rayons issus d'un point objet $A$ semblent provenir après réflexion du point $A^{\prime}$, symétrique de $A$ par rapport au plan du miroir : $A^{\prime}$ est l'image de $A$.
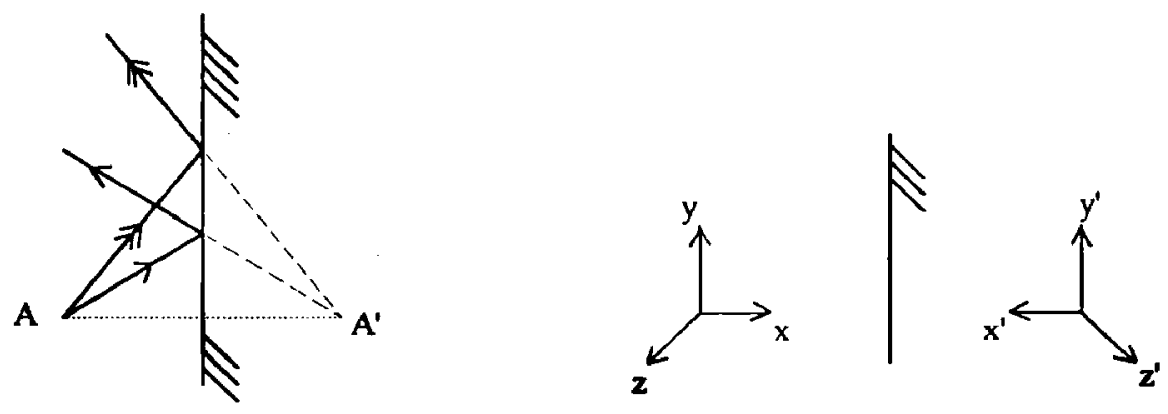

Lorsqu'on fait l'image d'un trièdre direct dans un miroir plan, il devient indirect ; pour un objet étendu, l'image dans le miroir n'est pas superposable à l'objet. On dit encore que la transformation de l'objet à l'image n'est pas congruente (pas décomposable en translations et rotations). C'est une propriété dont il faut tenir compte lorsqu'on fait de l'imagerie.

\subsubsection{Déplacement d'un miroir}

On considère un point objet fixe $A$ et on cherche la position de son image $A^{\prime}$ lorsqu'on déplace le miroir, en translation ou en rotation. On s'intéressera également à l'effet de ces déplacements sur le rayon émergent correspondant à un rayon incident fixe.

1.3.2.1 Translation du miroir. Quelle que soit la direction de translation du miroir, la quantité intéressante est le déplacement $d$ perpendiculaire au plan du miroir. L'image $A^{\prime}$ sera déplacée du double $2 \mathrm{~d}$ de cette quantité, perpendiculairement au plan du miroir.

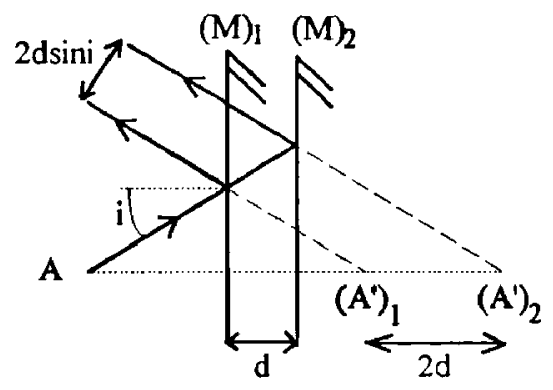

Pour un rayon incident fixe d'angle d'incidence $i$, le rayon émergent se déplace parallèlement à tui-même d'une quantité $2 d \sin i$. 
1.3.2.2 Rotation du miroir. On fait tourner le miroir plan $\mathrm{M}$ d'un angle $\alpha$ autour d'un axe $\Delta$ parallèle à son plan. Le nouveau plan $(M)_{2}$, déduit de la rotation, coupe le plan initial $(M)_{1}$ suivant $\Delta$. Représentons la réflexion dans un plan orthogonal à $\Delta$ et contenant l'objet $A$. Les droites intersections des miroirs avec ce plan font entre elles un angle $\alpha$. Les images $A_{1}^{\prime}$ et $A_{2}^{\prime}$ de $A$ correspondant aux deux positions $(M)_{1}$ et $(M)_{2}$ du miroir sont toutes dans ce plan d'apris la loi de Snell-Descartes.

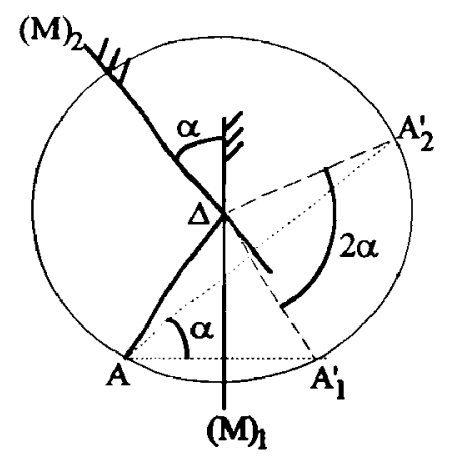

Les distances de $\mathrm{A}$ à $\Delta$, de $\mathrm{A}_{1}^{\prime}$ à $\Delta$ et de $\mathrm{A}_{2}^{\prime}$ à $\Delta$ sont égales. L'image s'est déplacée sur un cercle centré en $\Delta$ passant par $A$, d'un angle double $2 \alpha$.

Pour déterminer l'effet de la rotation sur le rayon réfléchi, on va utiliser le résultat précédent en considérant deux points $\mathrm{A}$ et $\mathrm{B}$ qui définissent le rayon incident. Le rayon émergent passe par les images $A^{\prime}$ et $B^{\prime}$ dans le miroir. Lorsque le miroir tourne d'un angle $\alpha, A^{\prime}$ et $B^{\prime}$ tournent d'un angle $2 \alpha$ autour de $\Delta$ : le rayon émergent tourne donc d'un angle $2 \alpha$.

1.3.2.3 Réglage de la direction d'un rayon à l'aide de miroirs plans. On utilise souvent dans un montage optique les miroirs plans pour modifier la direction d'un faisceau, par exemple pour superposer deux faisceaux, ou pour centrer un faisceau sur l'axe optique d'un système. Dans le cas le plus général où les directions initiales et finales sont quelconques, il va falloir 4 paramètres de réglage (une droite est définie par 4 paramètres dans l'espace à 3 dimensions : par exemple, les deux coordonnées d'un point d'intersection avec l'un des plans Oxy, Oyz ou Oxz et les deux coordonnées indépendantes d'un vecteur directeur unitaire).

Ceci ne peut être réalisé avec un seul miroir plan, puisque les droites ne sont pas forcément coplanaires. Il faut (et il suffit) deux miroirs plans et 4 réglages, qui sont en général uniquement des rotations car il est difficile de réaliser mécaniquement des translations pures.

Choisissons par exemple de doter chaque miroir de deux réglages en rotation. Les axes de rotation sont pris horizontal et vertical, ce qui permet de découpler les réglages dans les deux plans, et contenus dans le plan du miroir de sorte que le point d'impact du rayon incident ne se déplace quasiment pas lorsqu'on tourne le miroir. La figure montre ce qui se passe dans l'un des deux plans avec deux rotations. 


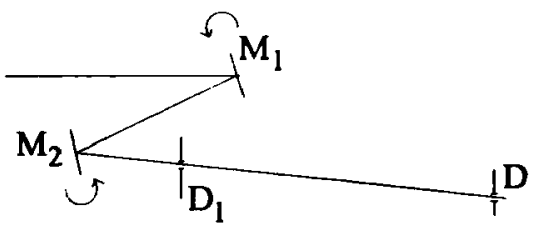

La procédure de réglage pour faire passer le faisceau à travers les deux diaphragmes $D_{1}$ et $D_{2}$ consiste à régler $M_{1}$ pour passer à travers $D_{1}$ puis $M_{2}$ pour passer à travers $D_{2}$, et quelques itérations de ces deux réglages, la procédure converge rapidement (d'autant plus vite que $\mathrm{M}_{2}$ est proche de $\mathrm{D}_{1}$ ). A noter que, les miroirs ayant une taille limitée, on a intérêt en pratique à les placer à peu près au bon endroit avant de tenter le réglage par rotation.

\subsubsection{Association de miroirs plans}

\subsubsection{Deux miroirs plans parallèles}

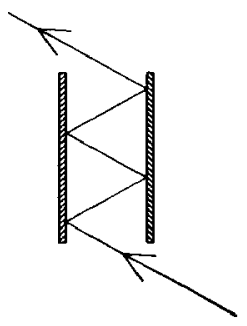

Les normales aux deux miroirs sont confondues. Le rayon reste dans un plan et se réfléchit sur les deux miroirs avec le même angle d'incidence. Il revient parallèle à lui-même après un nombre pair de réflexions. Ce type de système est utilisé par exemple comme cavité FabryPerot.

1.3.3.2. Deux miroirs plans faisant un angle $\alpha$. A partir de la situation précédente, on tourne l'un des miroirs d'un angle $\alpha$ :

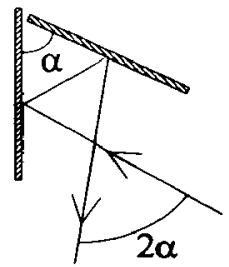

Le rayon émergent tourne donc de $2 \alpha$ par rapport à la situation précédente : il fait donc un angle de $2 \alpha$ avec le rayon incident. En particulier si les deux miroirs font un angle de $45^{\circ}$, le 
rayon émergent sortira orthogonal à l'incident, quelle que soit la direction de celui-ci. C'est le principe de l'équerre optique, souvent réalisé en utilisant un penta-prisme dont les faces sont traitées pour être réfléchissantes :

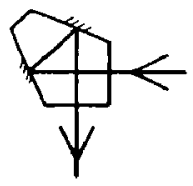

Cette équerre permet par exemple de fournir une référence horizontale en utilisant la réflexion sur la surface d'un liquide. Elle a aussi l'intérêt de ne pas inverser ni retoumer les images, et on l'utilise pour cela dans les viseurs d'appareil photo type "reflex".

1.3.3.3 Trois miroirs plans formant un coin de cube. Il s'agit d'un système formé de trois miroirs orthogonaux deux à deux :

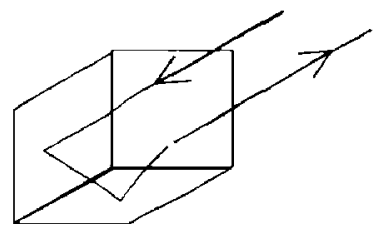

On montre facilement à partir des lois de Snell-Descartes que le rayon émergent ressort toujours parallèle au rayon incident. Un tel système est souvent utilisé à la place d'un miroir plan unique lorsqu'on ne peut régler l'orientation. Par exemple, de tels rétroréflecteurs ont été installés sur la Lune pour mesurer la distance Terre - Lune par mesure du temps d'aller-retour de la lumière ; ils sont également utilisés dans les bras d'un interféromètre de Michelson servant à des mesures interférométriques de déplacement, de sorte que le réglage de l'interféromètre soit conservé au cours du déplacement.

Les coins de cube, et de façon générale beaucoup d'associations de miroirs plans, peuvent être réalisées soit " en creux ", à partir de miroirs, soit sous forme de prismes réfléchissants dont nous allons parler dans le paragraphe suivant. Le choix entre les deux types de systèmes est lié à des contraintes de poids (systèmes plus légers avec des miroirs) ou de rigidité (en général meilleure avec des prismes).

\subsection{Application des lois de Descartes à l'étude des prismes}

Un prisme est un milieu transparent limité par des dioptres plans non parallèles. Dans ce cours nous nous limiterons au cas des prismes taillés dans un milieu homogène et isotrope. Il existe d'autres types de prismes formés de milieux biréfringents (Wollaston, Rochon, etc) permettant de modifier la polarisation de la lumière.

Nous distinguerons deux types de prismes : les prismes dispersifs, utilisés pour séparer les longueurs d'onde d'un spectre optique, mettent à profit la réfraction par un milieu d'indice $\mathbf{n}$ dépendant de la longueur d'onde ; les prismes à réflexion se rapprochent plutôt des associations de miroirs plans, et sont souvent utilisés pour le retournement et l'inversion des images. 


\subsection{Prismes dispersifs}

Un prisme dispersif typique est formé de deux dioptres plans formant un dièdre d'arête $\Delta$ et d'angle $A$ inférieur à $90^{\circ}$.

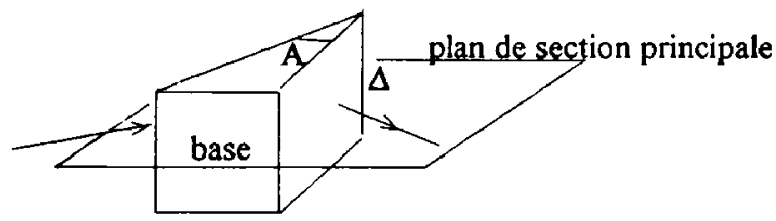

$A$ est appelé l'angle du prisme, la troisième face (verticale sur la figure) est la base, et un plan orthogonal à l'arête $\Delta$ est un plan de section principale du prisme. On ne s'intéressera dans la suite qu'à des rayons situés dans un plan de section principale.

\subsubsection{Formules du prisme : déviation d'un rayon incident}

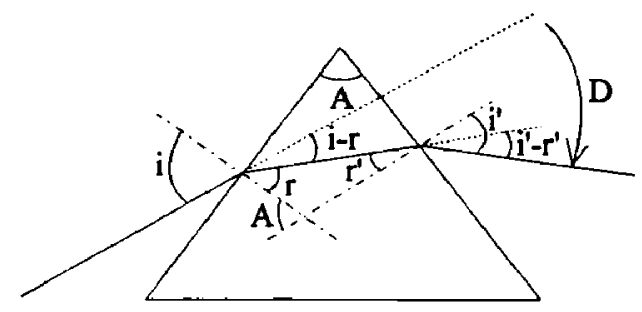

Les lois de Snell-Descartes et la figure ci-dessus permettent d'écrire les relations suivantes:

$$
\begin{gathered}
\sin i=n \sin r \\
\sin i^{\prime}=n \sin r^{\prime} \\
r+r^{\prime}=A \\
D=i-r+i^{\prime}-r^{\prime}=i+i^{\prime}-A
\end{gathered}
$$

Remarque : en ce qui concerne les signes, les angles sont pris tous positifs pour une configuration du type de celle de la figure ci-dessus, qui est la plus courante pour les prismes dispersifs. Si on applique ces relations au cas général, le cas $r^{\prime}<0$ par exemple correspondra à un rayon interne situé au-dessous de la normale au dioptre de sortie (cas d'un prisme d'angle A faible). Avec cette convention, les sens positifs des angles sont donc définis différemment à l'entrée et à la sortie, mais ceci présente l'avantage de garder la symétrie due au retour inverse de la lumière.

On peut tirer de ces relations l'expression de $D$ en fonction de $i$ mais il est plus facile de déterminer les propriétés du prisme en partant des quatre relations précédentes et en étudiant la dépendance de $\mathrm{D}$ avec l'angle $\mathrm{A}$, l'incidence $i$ et l'indice $n$ successivement. C'est ce que nous allons faire dans ce qui suit. On peut toutefois remarquer que l'expression de $D$ devient très 
simple dès lors que les angles $A$ et $i$ sont suffisamment faibles pour qu'on puisse linéariser les lois de réfraction. On obtient alors :

$$
D=(n-1) A
$$

1.4.1.2 Variation de la déviation avec l'angle du prisme $A$. Un raisonnement simple permet de trouver que $\mathrm{D}$ augmente quand $A$ augmente, à $i$ et $n$ fixés : si $A$ augmente, $r^{\prime}$ augmente, donc $i^{\prime}$ augmente (on peut aussi obtenir l'expression de $d D / d A$ en différentiant les quatre relations du prisme à $\boldsymbol{i}$ et $\boldsymbol{n}$ constants). La déviation $\mathrm{D}$, nulle pour $A$ nul, est donc forcément positive (avec la définition du sens de $D$ indiquée sur la figure). Autrement dit la déviation se fait vers la base du prisme.

1.4.1.3 Influence de l'angle d'incidence $i$. Conditions d'émergence d'un rayon. Nous nous sommes placés jusqu'ici dans le cas où le rayon incident ressort du prisme après deux réfractions. La première réfraction est toujours possible (il $\mathrm{y}$ a bien sûr aussi une partie réfléchie) puisqu'on passe d'un indice 1 à un indice $n$ plus grand. Par contre pour le deuxième dioptre verre/air, il peut y avoir réflexion totale, ce qui empêche l'émergence dủ rayon. Voyons les conditions dans lesquelles il y a émergence du rayon.

Le plus simple est de faire une figure représentant les zones accessibles à l'intérieur du prisme d'une part, pour les rayons entrants réfractés sous l'angle $r$ et d'autre part, pour les rayons d'angle $r^{\prime}$ pouvant émerger.

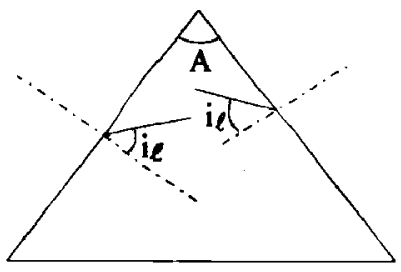

Les rayons pouvant émerger sont ceux qui sont à la fois inclus dans les deux zones angulaires grisées. On trouve déjà une condition nécessaire sur l'angle $A$ du prisme pour qu'il y ait un recouvrement entre ces deux zones:

$$
A \leq 2 i,
$$

On voit donc que pour un matériau d'indice $n=1.5$ par exemple, tout prisme dispersif devra avoir un angle au sommet inférieur à $2 \arcsin (1 / n)=84^{\circ}$.

Pour un angle $A$ remplissant cette condition et dans le cas de la figure où $A$ est plus grand que $i_{i}$, on détermine graphiquement les conditions sur l'angle d'incidence $i$ : 


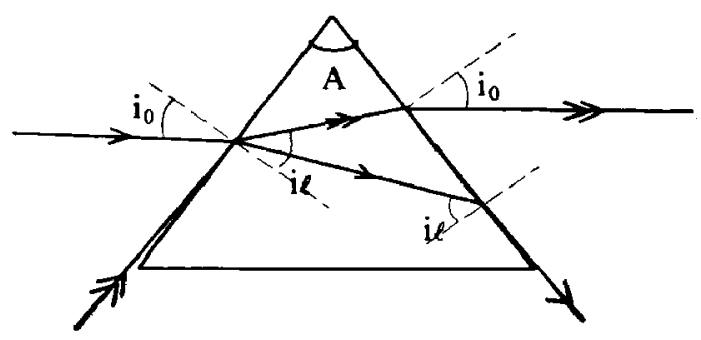

On trouve :

$$
\begin{gathered}
i_{0} \leq i \leq \pi / 2 \\
\text { avec } \sin i_{0}=n \sin \left(A-i_{1}\right)
\end{gathered}
$$

Compte tenu de la propriété de retour inverse de la lumière, la plage angulaire accessible est la même à l'entrée et à la sortie. Le cas où $A$ est plus faible que $i_{\text {q }}$ conduit à la même relation, mais cette fois $i_{0}$ est négatif, c'est-à-dire que la plage d'angle d'incidence accessible est supérieure à $90^{\circ}$.

1.4.1.4 Variation avec l'angle d'incidence i. Minimum de déviation. On s'intéresse ici à la variation de la déviation $D$ lorsqu'on fait varier l'angle d'incidence $i$ dans la plage où il y a émergence du rayon.

Une première remarque que l'on peut faire est que, du fait du retour inverse de la lumière, si l'angle d'incidence $i$ émerge avec un angle $i^{\prime}$, un rayon d'incidence $i$ ' va émerger du prisme sous un angle $i$. Si $i \neq i^{\prime}$, il existe donc deux angles d'incidence qui donnent la même déviation $D$. Entre ces deux valeurs d'angle d'incidence, la variation de $D$ est continue donc elle va forcément passer par un extremum (on peut montrer ensuite qu'il s'agit bien d'un minimum), et cet extremum ne peut se produire que lorsque les angles incident et émergent sont égaux. Dans ce cas, les relations du prisme donnent :

$$
r=\frac{A}{2} \quad i_{m}=\arcsin \left(n \sin \frac{A}{2}\right) \quad D_{m}=2 \arcsin \left(n \sin \frac{A}{2}\right)-\mathrm{A}
$$

La détermination expérimentale de ce minimum de déviation est aisée, à condition de disposer le prisme sur un support réglable en orientation par rapport au faisceau incident. On utilise souvent cette méthode pour déterminer l'indice d'un matériau grâce à l'expression suivante :

$$
n=-\frac{\sin \left(A+D_{m}\right) / 2}{\sin A / 2}
$$

1.4.1.5 Dispersion. Exemples de prismes dispersifs. A partir des quatre relations du prisme, on peut déjà voir que la déviation augmente avec l'indice : à $i$ donné, si $n$ augmente, $r$ diminue, donc $r^{\prime}$ augmente et $i^{r}$ augmente. Or dans la plupart des matériaux, l'indice diminue quand la longueur d'onde augmente (dispersion dite normale). On voit donc que le prisme va dévier la lumière bleue plus fortement que la lumière rouge. 
Par exemple un prisme en verre BK7 (encore appelé crown) d'angle $A=60^{\circ}$, éclairé en lumière blanche sous un angle d'incidence de $40^{\circ}$, va dévier le bleu $\left(\lambda=400 \mathrm{~nm}, \mathrm{n}_{\mathrm{BK} 7}=1.530\right)$ d'un angle $D_{B}=41.8^{\circ}$ et le rouge $(\lambda=650 \mathrm{~nm}, \mathrm{n}=1.516)$ d'un angle $D_{R}=40.2^{\circ}$.

Remarque: un autre matériau couramment utilisé est le flint dont la dispersion est plus grande mais la qualité optique moins bonne.

Signalons enfin qu'à part le prisme dispersif à deux dioptres décrit plus haut il existe deux autres formes utiles de prismes dispersifs, dont la particularité est de présenter une valeur du minimum de déviation $\mathrm{D}_{m}$ indépendante de l'indice donc de la longueur d'onde. Cette valeur $\mathrm{D}_{\mathrm{m}}$ vaut $90^{\circ}$ pour le Pellin-Broca et $60^{\circ}$ pour le prisme d'Abbe, et elle correspond dans les deux cas à des angles réfractés de $30^{\circ}$, identiques à l'entrée et à la sortie. La différence avec le prisme dispersif précédent vient du fait quil y a une réflexion totale, ce qui conduit notamment à des angles $i$ et $i^{\prime}$ de même sens.

Pour faciliter la compréhension de la marche des rayons, nous avons représenté ces deux prismes comme la juxtaposition de trois prismes dont les limites sont indiquées en pointillés :

prisme de Pellin-Broca

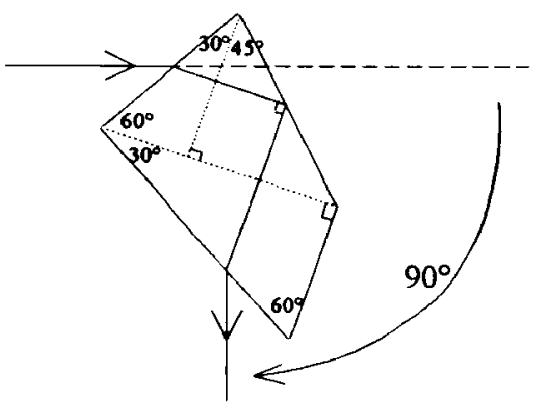

prisme d'Abbe

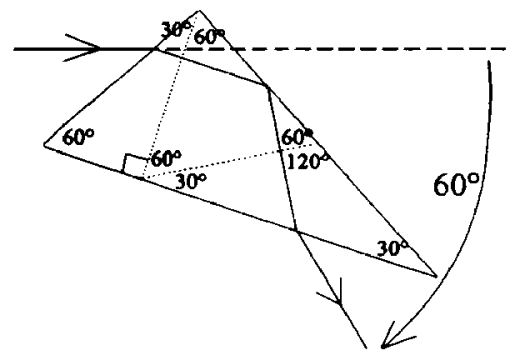

\subsubsection{Prismes réfléchissants}

Nous allons maintenant voir un type complètement différent de prismes, utilisant les propriétés de réflexion à l'intérieur des prismes, et exempts de dispersion. Ces prismes seront utilisés soit pour dévier des faisceaux, soit pour modifier l'orientation d'images (appareil photo reflex, jumelles), parfois les deux. Ils se rapprochent plus par conséquent des associations de miroirs. Ils ont sur les miroirs l'avantage d'être monolithiques, ce qui fait qu'ils sont réglés par construction et moins sensibles aux vibrations. De plus, dans le cas de prismes à réflexion totale, les facteurs de réflexion sont élevés sur une très large plage de longueur d'onde.

La première condition à remplir pour qu'un prisme réfléchissant remplisse son rôle est d'éviter ou de réduire la dispersion, qui est a priori liée au phénomène de réfraction à l'entrée et à la sortie du prisme. Voyons comment on peut remplir cette condition sur un exemple de prisme réfléchissant simple. Il s'agit d'un prisme isocèle d'angle au sommet $A$ qui ressemble fort au prisme dispersif vu plus haut sauf en ce qui concerne le trajet utilisé : 


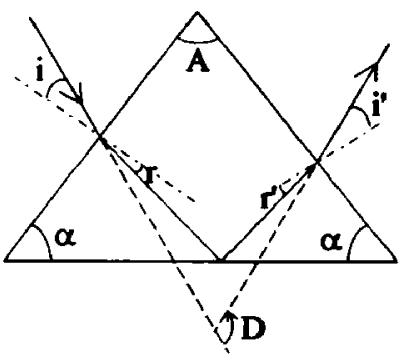

La symétrie de ce prisme isocèle conduit à l'égalité des angles $r$ et $r^{\prime}$ d'une part et $i$ et $i^{\circ}$ d'autre part et on obtient pour la déviation :

$$
D=2 i+A,
$$

en prenant tous les angles positifs sur la figure (attention ce n'est plus la même convention de signe que pour le prisme dispersif). $D$ est donc indépendante de l'indice donc de la longueur d'onde. Par opposition au prisme dispersif, un tel prisme sera appelé prisme achromatique.

Remarque : la réflexion interne sur la base du prisme peut soit être une réflexion totale (ce qui limite la plage d'angles d'incidences utilisables), soit être obtemue par métallisation de la surface du prisme.

On peut interpréter la propriété d'achromatisme du prisme ci-dessus par une autre méthode. Traçons, par symétrie par rapport au plan du miroir constitué par la base du prisme, le trajet symétrique des trajets avant réflexion:

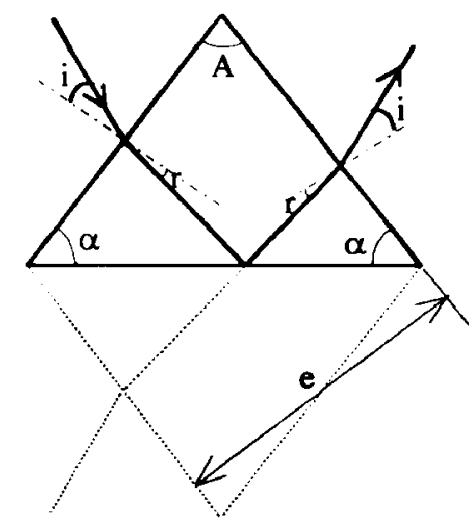

Tout se passe comme si on traversait une lame de verre à faces planes et parallèles d'épaisseur $e$ et d'indice $n$, que l'on obtient en complétant le prisme par son symétrique à travers le miroir. On retrouve que l'angle en sortie est égal à l'angle à l'entrée. Si l'on anticipe sur la formation des images dans l'approximation de Gauss (cf partie 3), on obtient que, comme pour une lame à faces planes et parallèles, l'image d'un petit objet à travers un tel prisme sera translatée de $e(n-1) / n$, dans une direction normale à la face de sortie du prisme, par rapport à l'image de cet objet dans le miroir plan. 
Il existe d'autres formes plus ou moins complexes de prismes achromatiques, où l'on utilise comme pour le prisme isocèle des propriétés de symétrie pour éviter la dispersion. Les figures qui suivent montrent quelques-uns des nombreux prismes achromatiques utilisés, pour lesquels nous mentionnons brièvement les propriétés principales.
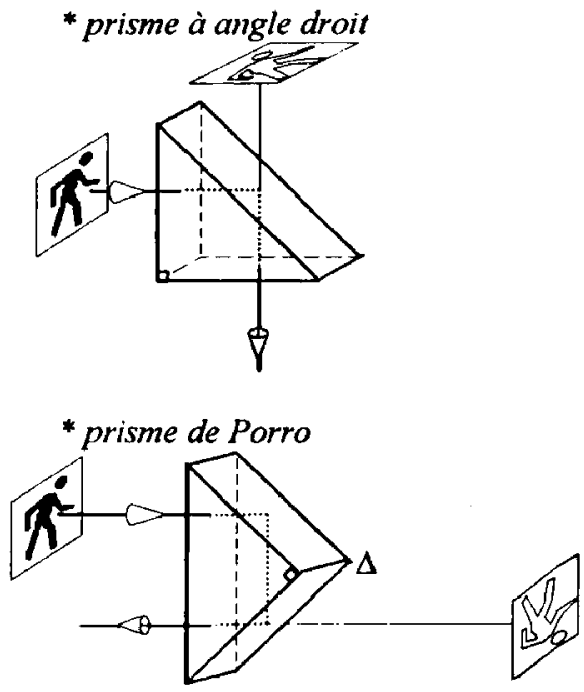

* prisme de Dove

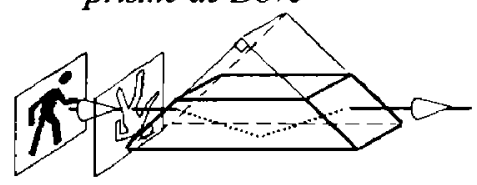

Pour un observateur fixe par rapport au sens de propagation de la lumière, le haut et le bas sont inversés, mais la droite et la gauche sont conservées.

Ce prisme, identique au précédent, donne dans cette configuration une image superposable à l'objet par une rotation de $180^{\circ}$ autour de $\Delta$.

Il s'agit à nouveau d'un prisme à angle droit, dont une partie est coupée pour alléger et réduire l'encombrement. Il est utilisé en lumière parallèle.

\section{FORMATION DES IMAGES : Stigmatisme rigoureux ou approché}

Nous allons maintenant nous intéresser à des rayons particuliers, ceux qui sont issus d'un objet et viennent après passage dans un système optique former une image.

Les systèmes optiques que nous considérerons dans la suite seront presque toujours constitués de milieux homogènes et isotropes, séparés par des dioptres (ce sont les systèmes dioptriques, comme la lentille, le microscope, la lunette de Galilee) et parfois aussi par des surfaces réfléchissantes (systèmes catadioptriques, comprenant au moins un miroir). Il s'agira pour la plupart de systèmes centrés, c'est-à-dire possédant un axe de symétrie (dans ce cas, le rayon confondu avec l'axe ne sera pas dévié).

Nous verrons que la condition pour qu'un système optique donne d'un point objet un point image parfait (stigmatisme rigoureux) est très contraignante et qu'elle n'est pas satisfaite pour la plupart des systèmes optiques simples. Il existe cependant certains systèmes qui sont rigoureusement stigmatiques pour un couple de points objet/image particulier, et de plus certains d'entre eux restent stigmatiq̨ues de façon approchée au voisinage de ces points 
particuliers. Ces systèmes sont très utiles lorsqu'on veut observer de très petits objets avec une grande ouverture, typiquement lorsqu'on observe une étoile avec un télescope ou une cellule au microscope.

\subsection{Stigmatisme rigoureux}

\subsubsection{Définition}

Un système optique est rigoureusement stigmatique pour un couple de points $A$ et $A^{\prime}$ si tous les rayons issus de $A$ se coupent en $A^{\prime}$ après traversée du système optique. $A^{\prime}$ est alors l'image parfaite de l'objet $A$ par le système optique, et vice versa si l'on inverse le sens de propagation de la lumière ; on dit que $\mathrm{A}$ et $\mathrm{A}^{\prime}$ sont conjugués par ce système.

Rappel: n'oublions pas que dans un système optique réel, il y a toujours de la diffraction liée au diamètre fini du système optique. L'image parfaite d'un objet ponctuel sera donc une figure de diffraction (tache d'Airy dans le cas d'une diaphragmation circulaire).

\subsubsection{Condition en termes de chemin optique}

En termes de chemin optique, le stigmatisme rigoureux se traduit par le fait que le chemin optique entre les points $\mathrm{A}$ et $\mathrm{A}^{\prime}$ est indépendant du rayon choisi : $\quad L\left(\mathrm{AA}^{\prime}\right)=c s t e$

Il suffit pour démontrer ceci de considérer deux rayons proches issus de $\mathrm{A}$ : la condition de stigmatisme nous dit qu'ils passeront par $A^{\prime}$, et le principe de Fermat que la variation de chemin optique est nulle. De proche en proche, on montre done que tous les rayons issus de $A$ correspondent au même chemin optique entre $\mathrm{A}$ et $\mathrm{A}^{\prime}$.

\subsubsection{Condition en termes de surface d'onde}

On peut grâce au théorème de Malus-Dupin associer une surface d'onde aux faisceaux de rayons. Pour un objet à distance finie, la surface d'onde objet associée est une calotte sphérique. S'il est à l'infini, la surface d'onde est un plan. De même pour l'image, si tous les rayons convergent en un point image, la surface d'onde image sera une calotte sphérique (un plan si l'image est à l'infini). Le stigmatisme rigoureux signifie donc que le système optique transforme une surface d'onde objet sphérique ou plane en une surface d'onde image sphérique ou plane. Cette propriété est souvent utilisée pour caractériser la qualité d'un système optique. On envoie une onde plane ou sphérique à l'entrée d'un système, et on mesure la déformation de la surface d'onde émergente par rapport à une sphère parfaite, en général par une méthode interférométrique (interféromètre de Twyman-Green, interféromètre Zygo).

\section{1.4 Objets et images réels et virtuels}

Un système optique est limité par une face d'entrée $S_{1}$ et une face de sortie $S_{2}$. Nous représenterons en général la lumière se propageant de gauche à droite. Un objet est réel s'il se trouve à gauche de la face d'entrée $S_{1}$, virtuel s'il se trouve à droite. Une image est réelle si elle se trouve à droite de la face de sortie $S_{2}$, virtuelle sinon.

Les objets et images virtuels ne correspondent plus à l'intersection des rayons lumineux, mais à lintersection du prolongement de ces rayons. 


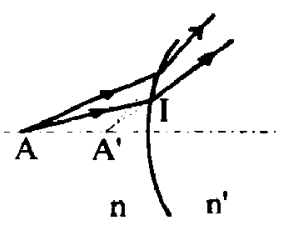

Pour le calcul d'un chemin optique, les trajets virtuels seront comptés négativement. Par exemple dans le cas d'un dioptre séparant deux milieux d'indice $n$ et $n^{\prime}$ (figure ci-dessus), le chemin optique de $\mathrm{A}$ à $\mathrm{A}^{\prime}$ s'écrit :

$$
L=n \overline{\mathrm{AI}}+n^{\prime} \overline{\mathrm{LA}^{\prime}} \text {, où } \overline{\mathrm{LA}^{\prime}} \text { est négatif. }
$$

Notons également que l'indice affecté au trajet virtuel (IA') est bien celui du milieu image $n^{\prime}$, bien que l'intersection des rayons émergents se trouve géométriquement à gauche du dioptre de la figure. En fait pour la suite de la propagation de ce rayon, tout se passe comme $s^{\prime} i l$ n'y avait qu'un milieu d'indice $n^{\prime}$ et un point objet en $A^{\prime}$ dans ce milieu.

Les figures qui suivent illustrent quelques cas de couples objet/image de différentes natures pour des systèmes centrés (le rayon confondu avec l'axe de symétrie n'est pas dévié).
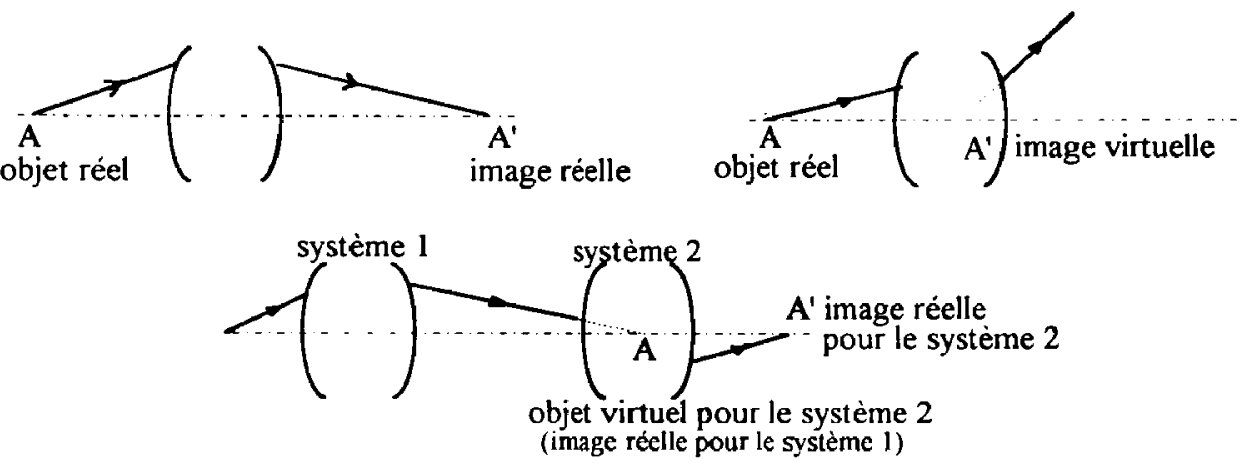

\subsection{Exemples de systèmes qui ne sont pas rigoureusement stigmatiques}

Il est intéressant de se rendre compte que la plupart des systèmes simples formés d'une ou deux surfaces planes ou sphériques ne remplissent pas la condition de stigmatisme rigoureux. Nous allons le voir sur deux exemples : le dioptre plan et le miroir sphérique.

Prenons le cas du dioptre plan séparant l'air d'indice 1 du verre d'indice 1.5. Considérons un objet à distance finie $\mathrm{A}$ et cherchons son image $\mathrm{A}^{\prime}$ par le dioptre. Le rayon passant par $\mathrm{A}$ et orthogonal au dioptre n'est pas dévié. Calculons l'intersection de ce rayon avec un autre rayon issu de $\mathrm{A}$ et faisant un angle d'incidence $\mathrm{i}$ avec le dioptre. 


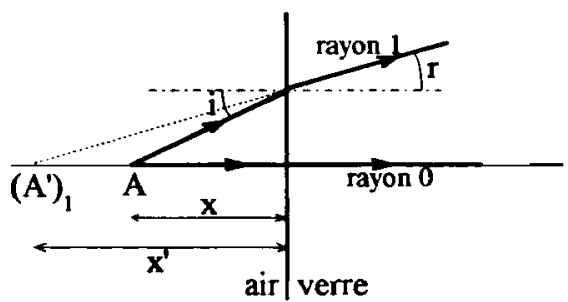

(1) (n)

En utilisant la loi de Snell-Descartes de la réfraction, on peut exprimer la position $x^{\prime}$ de l'intersection $\left(A^{\prime}\right)_{1}$ des rayons 0 et 1 :

$$
x^{\prime}=x \frac{\tan \mathrm{i}}{\tan \mathrm{r}}=\mathrm{nx} \sqrt{\frac{1-\sin ^{2} i / n^{2}}{\cos ^{2} i}}=n x \sqrt{1+\tan ^{2} \mathrm{i}\left(1-\frac{1}{n^{2}}\right)}
$$

On voit donc qu'en général la position du point d'intersection avec l'axe va dépendre de $i$, donc du rayon choisi : il n'y a pas stigmatisme rigoureux. On remarque que $A^{\prime}$ se rapproche du dioptre lorsque $i$ diminue, et que la distance $x^{\prime}$ tend vers $n x$ quand $i$ tend vers zéro.

La figure suivante montre le tracé exact (à partir des lois de Snell-Descartes) d'un grand nombre de rayons issus d'un objet à distance finie placé devant un dioptre plan.

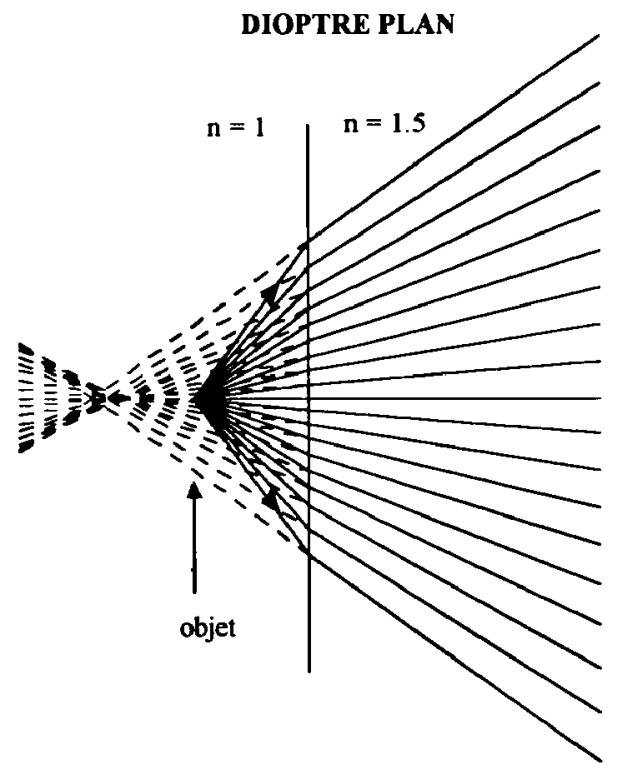


Un autre exemple simple est le cas d'un objet à l'infini sur l'axe d'un miroir sphérique :

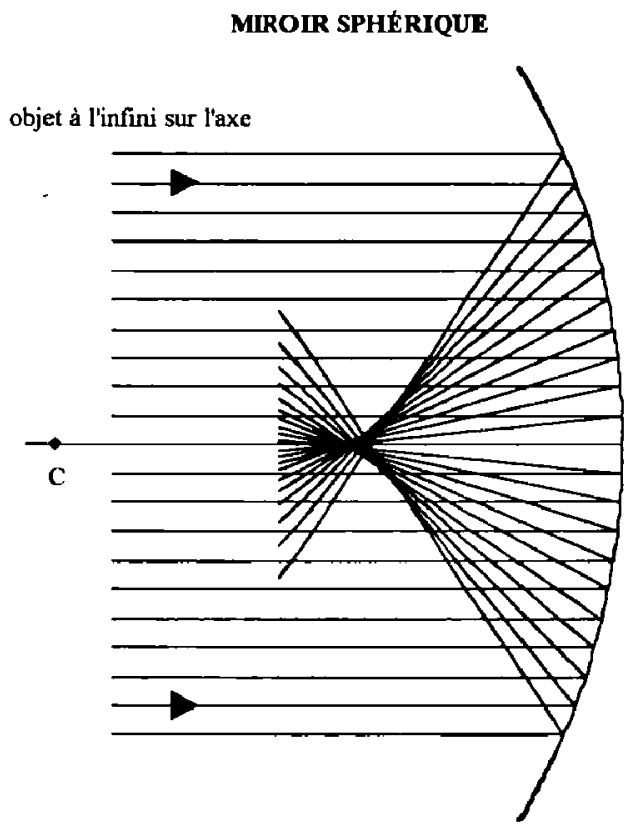

Dans ce type de situations, on n'obtient nulle part d'image parfaite. L'image "imparfaite" (entachée d'aberrations) correspond à la répartition d'énergie dans un plan d'observation orthogonal à l'axe du système. Cette tache image dépend du plan d'observation.

Ces deux exemples permettent déjà de se rendre compte que les aberrations sur l'image augmentent avec l'angle d'incidence sur les dioptres ou les miroirs. On utilisera donc souvent des systèmes optiques au voisinage de leur axe.

\subsection{Systèmes rigoureusement stigmatiques}

Bien que la majorité des systèmes optiques ne soient pas rigoureusement stigmatiques, il existe cependant quelques cas particuliers utiles de systèmes qui remplissent la condition de stigmatisme rigoureux pour des couples de points conjugués particuliers. Nous donnons ici pour mémoire les conjugaisons rigoureusement stigmatiques les plus courantes.

\subsubsection{Miroirs}

- Le miroir plan est stigmatique pour tous les points.

- Le miroir sphérique est stigmatique pour son centre de courbure : ceci est utilisé par exemple pour augmenter l'efficacité d'une source d'éclairage, on augmente l'angle solide utile en plaçant la lampe au centre de courbure du miroir. 
- Le miroir elliptique est stigmatique pour ses foyers. (noter qu'il ne faut pas confondre le foyer de la conique et le foyer du système optique).

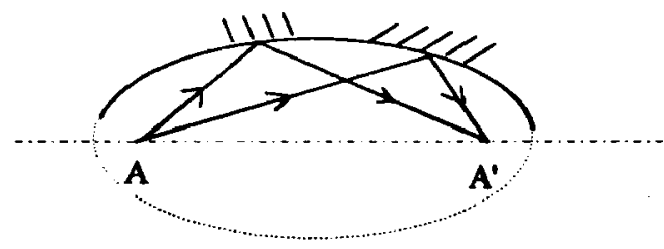

Ce type de miroir est utilisé par exemple pour le pompage des barreaux laser type Nd:YAG par lampe à décharge, la lampe étant placée à l'un des foyers de l'ellipse et le barreau laser à l'autre (la section est elliptique, l'autre dimension étant invariante par translation). Si l'on souhaite augmenter la puissance de pompage en utilisant deux lampes au lieu d'une, on choisit alors un miroir double elliptique : les deux ellipses ont un foyer commun, où l'on vient placer le barreau, les deux lampes se trouvant aux deux autres foyers :

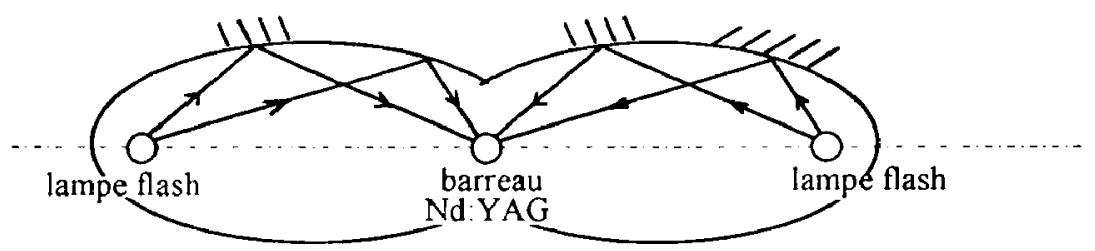

- Le miroir hyperbolique est stigmatique pour ses foyers.

- Le miroir parabolique est stigmatique pour le couple de points infini - foyer. (dans ce cas foyer de la conique ei foyer du systeme optiques sont corffondus).

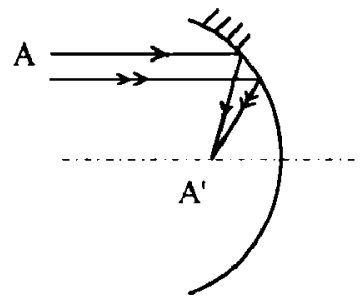

Il faut signaler que ce type de miroir devient vite fortement aberrant quand on s'éloigne du foyer idéal. Il est utilisé par exemple pour collimater une source de forte intensité (on place la source en $A^{\prime}$ de façon à obtenir un faisceau de sortie parallèle), pour collecter l'énergie solaire, et aussi très souvent en astrononie comme miroir primaire.

\subsubsection{Dioptres}

- Le dioptre plan n'est stigmatique que pour un point objet à l'infini. 
- Le dioptre sphérique est stigmatique pour son centre et pour les points d'Young-Weierstrass.

Ces points particuliers sont souvent utilisés dans les objectifs de microscope. On peut déterminer leurs positions à partir d'une figure :

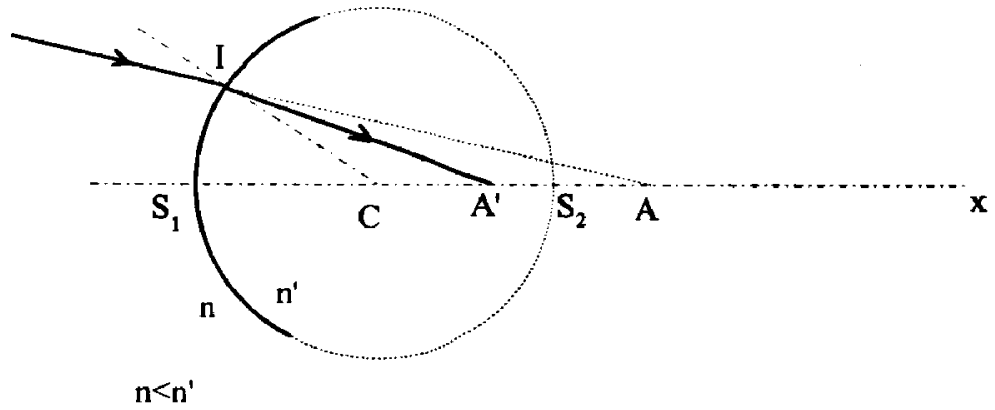

La condition de stigmatisme s'écrit pour ces points : $n \overline{\mathrm{AI}}+n^{\prime} \overline{\mathrm{IA}^{\prime}}=0 . \overline{\mathrm{AI}}$ et $\overline{\mathrm{IA}^{\prime}}$ sont donc de signe opposé, l'objet et l'image sont de nature différente, l'un réel, l'autre virtuel.

Par ailleurs, tout rayon passant par le centre du dioptre (donc suivant un diamètre) n'étant pas dévié, les couples de points conjugués se trouvent forcément sur le même diamètre. Par symétrie autour de $C$, les points d'Young-Weierstrass vont décrire un cercle suivant la zone utilisée du dioptre.

A partir de la condition de stigmatisme et de la figure, on montre que la position des points est donnée par:

$$
X=\overline{\mathrm{CA}}=R \frac{n^{\prime}}{n} \quad \text { et } \quad X^{\prime}=\overline{\mathrm{CA}^{\prime}}=R \frac{n}{n^{\prime}}
$$

D'autres formes particulières de dioptres permettent de conjuguer de façon rigoureusement stigmatique un objet à l'infini et une image à distance finie, avec des surfaces hyperboliques ou elliptiques.

\subsection{Stigmatisme approché au voisinage des points stigmatiques}

Nous remarquons en conclusion du paragraphe précédent que les systèmes rigoureusement stigmatiques ont souvent des formes géométriques complexes, et surtout qu'ils ne sont stigmatiques que pour un couple de points bien précis, ce qui limite leur intérêt pour former des images d'objets étendus. Toutefois, il va nous falloir faire une distinction parmi ces systèmes rigoureusement stigmatiques suivant qu'ils détériorent plus ou moins rapidement les images lorsqu'on se déplace au voisinage de ces points idéaux.

En fait, il n'est pas toujours nécessaire d'être dans des conditions de stigmatisme rigoureux. En effet, d'autres phénomènes vont limiter la qualité de l'image, telles la diffraction ou la résolution du détecteur (fixée par la taille d'une cellule de la rétine, ou le grain d'un film, ou la dimension d"un élément de barrette ou de matrice $\mathrm{CCD}$ ). Il faut donc adapter la qualité du système optique à l'ensemble de l'expérience et se fixer des conditions de stigmatisme approchè.

Le stigmatisme approché dont nous allons parler dans ce chapitre concerne des systèmes déjà rigoureusement stigmatiques pour un couple de points particuliers. Il s'agit de rechercher 
les conditions pour lesquelles un tel système restera stigmatique pour des points très voisins des points idéaux, tout en s'autorisant des rayons aussi inclinés sur l'axe que l'on veut. On fait donc une approximation sur la taille des objets à observer, mais pas sur l'ouverture des systèmes. Le cas le plus intéressant en pratique correspond au maintien du stigmatisme de façon approchée dans le plan orthogonal à l'axe optique (aplanétisme, condition d'Abbe).

Il existe un autre moyen, plus radical, qui permet de rendre stigmatique tout système optique pour tous les points objets : on limite à la fois la taille des objets mais aussi les angles d'incidence sur les surfaces optiques. C'est l'approximation de Gauss, qui sera traitée dans la partie 3.

\subsubsection{Comventions de signe}

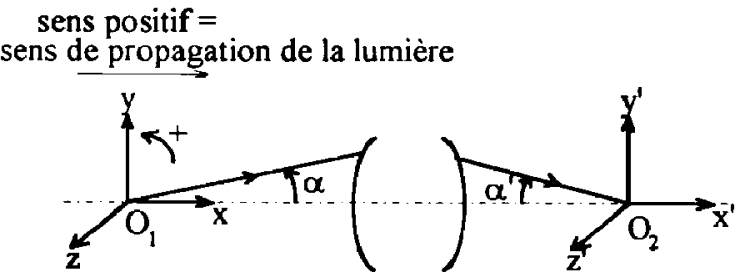

Il s'agit ici d'un système centré d'axe noté $x$ dans l'espace objet et $x^{\prime}$ dans l'espace image. L'espace objet est décrit dans un repère $O_{1} x y z$, l'espace image dans un repère $\mathrm{O}_{2} x^{\prime} y^{\prime} z^{\prime}$. Le sens positif de $x$ et $x^{\prime}$ est défini dans le sens de propagation de la lumière (attention au cas du miroir). Les deux trièdres sont directs, leurs origines $O_{1}$ et $O_{2}$ ne sont pas forcément conjuguées. Les angles sont comptés algébriquement entre $-90^{\circ}$ et $+90^{\circ}$, de l'axe vers le rayon (sur la figure $\alpha$ est positif et $\alpha^{*}$ est négatif).

\subsubsection{Stigmatisme approché dans un élément de volume (pour un système optique de révolution)}

On considère un système rigoureusement stigmatique pour un couple de points $A$ et $A^{\prime}$. On cherche à écrire la condition pour que deux points $M$ et $\mathbf{M}^{\prime}$, respectivement proches de $A$ et $A^{\prime}$, vérifient la condition de stigmatisme. Un rayon quelconque issu de $M$ et passant par un point $I$, va sortir en passant par $\mathbf{M}^{\prime}$ et le chemin optique (MIM') doit être indépendant de $\mathrm{I}$.

Comme $\mathbf{M}$ et $\mathbf{M}^{\prime}$ sont très proches de $\mathbf{A}$ et $\mathbf{A}^{\prime}$, et que $\mathrm{AIA}^{\prime}$ est un chemin suivi par la lumière, le chemin optique (MIM') peut s'exprimer en fonction du chemin (AIA') issu de $A$ et passant par le même point I, sous la forme :

$$
\left(\mathrm{MIM}^{\prime}\right)=\left(\mathrm{AIA}^{\prime}\right)+n^{\prime} \mathbf{u}^{\prime} \cdot \mathbf{A}^{\prime} \mathbf{M}^{\prime}-n \mathbf{n} . \mathbf{A M}
$$

où $n$ et $n^{\prime}$ sont les indices des milieux objet et image, et $\mathbf{u}$ et $\mathbf{u}^{\prime}$ sont les vecteurs unitaires portés par les rayons incident issu de $A$ et émergent en $A^{\prime}$. Notons que cette expression est 
correcte à des termes du second ordre en $\mathrm{A}^{\prime} \mathrm{M}$ ' et en $\mathrm{AM}$ près. C'est donc dans cette évaluation du chemin qu'apparaît le stigmatisme approché

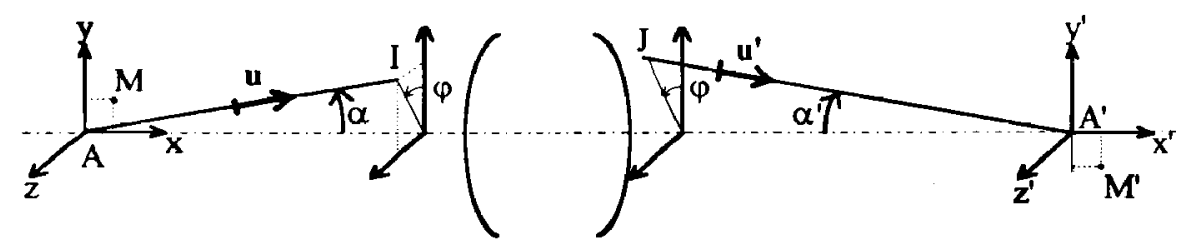

Le système étant rigoureusement stigmatique pour $A$ et $A^{\prime}$, le chemin (AIA') est indépendant de I. La condition (MIM') indépendant de I peut donc s'écrire :

$$
n^{\prime} \mathbf{u}^{\prime} . \mathbf{A}^{\prime} \mathbf{M}^{\prime}-n \mathbf{u} . \mathbf{A M}=\operatorname{cste}(\text { independante } d u \text { point } I)
$$

Ecrivons cette condition en fonction des coordonnées des points $\mathbf{M}$ et $\mathbf{M}^{\prime}$. On choisit un repère Axyz de sorte que $M$ se trouve dans le plan Axy. Par symétrie par rapport à ce plan, on montre que $\mathrm{M}^{\prime}$ est aussi dans ce plan. On peut également remarquer que la symétrie par rapport au plan (AIA') impose que u et $\mathbf{u}^{\prime}$ soient forcément dans un même plan contenant l'axe, que l'on repère par son angle $\varphi$ par rapport au plan de figure.

Remarque : cette propriété utilise le fait que le rayon incident AI est dans un plan contenant l'axe. Notez qu'un rayon comme MI n'est pas contenu dans un tel plan.

Les coordonnées des vecteurs $\mathbf{A} \mathbf{M}, \mathbf{A}^{\prime} \mathbf{M}^{\prime}$, $\mathbf{u}$ et $\mathbf{u}^{\prime}$ peuvent finalement s'écrire :

$$
\mathbf{A M}\left[\begin{array}{l}
d x \\
y \\
0
\end{array}\right] \quad \mathbf{A}^{\prime} \mathbf{M}^{\prime}\left[\begin{array}{c}
d x^{\prime} \\
y^{\prime} \\
0
\end{array}\right] \mathbf{u}\left[\begin{array}{c}
\cos \alpha \\
\sin \alpha \cos \varphi \\
\sin \alpha \sin \varphi
\end{array}\right] \quad \mathbf{u}^{\prime}\left[\begin{array}{c}
\cos \alpha^{\prime} \\
\sin \alpha^{\prime} \cos \varphi \\
\sin \alpha^{\prime} \sin \varphi
\end{array}\right]
$$

Remarque : les quantités dx et y sont toutes deux du premier ordre. Cette notation permet d'éviter par la suite la confusion entre $x$, coordonnées d'un point objet le long de l'axe par rapport à l'origine du repère et dx, petit déplacement autour d'un tel point.

La condition devient :

$$
\left.n^{\prime} d x^{\prime} \cos \alpha^{\prime}-n d x \cos \alpha+\left(n^{\prime} y^{\prime} \sin \alpha^{\prime}-n y \sin \alpha\right) \cos \varphi=\text { cste (indépendante de } \alpha \text { et } \varphi\right)
$$

Elle doit être vérifiée pour tout rayon, donc pour tout $\varphi$, d'où :

$$
\begin{gathered}
n^{\prime} y^{\prime} \sin \alpha^{+}=n y \sin \alpha \\
n^{\prime} d x^{\prime} \cos \alpha^{\prime}-n d x \cos \alpha=\text { cste }
\end{gathered}
$$

La constante peut être évaluée pour le rayon sur l'axe $\left(\alpha=\alpha^{\prime}=0\right)$, elle vaut $n^{\prime} d x^{\prime}-n d x$. On peut alors récrire les deux équations sous la forme : 


$$
\begin{array}{ll}
n^{\prime} y^{\prime} \sin \alpha^{\prime}=n y \sin \alpha & \text { relation d'Abbe } \\
n^{\prime} d x^{\prime} \sin ^{2} \frac{\alpha^{\prime}}{2}=n d x \sin ^{2} \frac{\alpha}{2} & \text { relation d'Herschel }
\end{array}
$$

\subsubsection{Incompatibilité des relations d'Abbe et Herschel dans le cas général}

Choisissons un point $M$ qui n'est ni sur l'axe $(d x \neq 0)$ ni dans le plan de front de $A(y \neq 0)$. Les relations d'Abbe et d'Herschel peuvent alors s'écrire :

$$
\begin{gathered}
\frac{y^{\prime}}{y}=\frac{n \sin \alpha}{n^{\prime} \sin \alpha^{\prime}} \\
\frac{d x^{\prime}}{d x}=\frac{n \sin ^{2} \alpha / 2}{n^{\prime} \sin ^{2} \alpha^{\prime} / 2}
\end{gathered}
$$

Pour un point $M$ et son image $M^{\prime}$ fixés, ces deux quantités doivent être constantes quel que soir le rayon choisi, donc quel que soit $\alpha$. En remplaçant dans la première relation $\sin \alpha$ par $2 \sin \alpha / 2 \cdot \cos \alpha / 2$ (idem pour $\sin \alpha^{\prime}$ ), on trouve que le rapport $\left|\cos (\alpha / 2) / \cos \left(\alpha^{\prime} / 2\right)\right|$ doit lui aussi être constant en valeur absolue. Il est donc égal à la valeur qu'il prend lorsque $\alpha=0$, auquel cas $\alpha^{\prime}=0$ par symétrie de révolution, c'est-à-dire :

$$
\begin{gathered}
\left|\frac{\cos \alpha / 2}{\cos \alpha^{\prime} / 2}\right|=\operatorname{cste}=\frac{\cos (\alpha / 2=0)}{\cos \left(\alpha^{\prime} / 2=0\right)}=1 \\
\alpha= \pm \alpha^{\prime} \quad \text { (angles compris entre }-90^{\circ} \text { et } 90^{\circ} \text { ) }
\end{gathered}
$$

On voit donc que si on s'autorise des rayons fortement inclinés par rapport à l'axe, les seuls points stigmatiques au voisinage desquels le stigmatisme peut être conservé de façon approchée dans toutes les directions sont ceux pour lesquels le grandissement angulaire vaut 1 ou - 1. Le grandissement transversal est limité au rapport des indices des milieux extrêmes (1 lorsque les milieux sont identiques), ce qui est très limitatif. Le miroir plan rentre dans ce cadre pour tous les points objets, et le miroir sphérique pour son centre de courbure

Nous allons voir maintenant que la situation est moins contraignante si on se contente de conserver le stigmatisme soit dans un plan de front (observation de petits objets), soit le long de l'axe (observation avec une petite profondeur de champ).

Remarque : dans le cas où l'on se limite non seulement à des petits objets ( $y$ et dx petits) mais aussi à des angles faibles ( $\alpha$ et $\alpha$ ' petits), le rapport des cosinus est égal d̀ $l \dot{a}$ des termes du second ordre près même si $\alpha$ et $\alpha$ ' he sont pas égaux. C'est l'approximation de Gauss. Dans ces conditions les relations d'Abbe et d'Herschel donnent alors les relations entre les grandissements $g_{y}=y^{\prime} / y, g_{\alpha}=\alpha^{\prime} / \alpha, g_{x}=d x^{\prime} / d x$, au voisinage des points $A$ et $A^{\prime}$ :

$$
g_{y} g_{\alpha}=\frac{n}{n^{\prime}} \quad g_{x} g_{\alpha}^{2}=\frac{n}{n^{\prime}} \quad g_{x}=\frac{n^{\prime}}{n} g_{y}^{2} \quad g_{y}=g_{x} g_{\alpha}
$$




\subsubsection{Aplanétisme : condition d'Abbe}

On reprend les conditions précédentes dans le cas où $\mathbf{M}$ appartient au plan orthogonal à l'axe contenant $\mathrm{A}(d x=0)$. La relation d'Herschel, qui doit être vraie quel que soit le rayon incident donc pour tout $\alpha$, impose que $d x^{\prime}=0$ : l'image doit donc être dans le plan orthogonal contenant $\mathrm{A}^{\prime}$. Montrons que cette condition est forcément remplie au ler ordre en y:

Puisqu'on se limite au ler ordre, l'expression la plus générale pour dx' s'écrit $d x^{\prime}=a y+b$. On sait que si $M=A, M^{\prime}=A^{\prime}:$ b est donc nul. De plus la symétrie de révolution du système impose que si $M_{1}$, est symétrique de $M$ par rapport à l'axe (soit $y_{I}$ $=-y$ ), son image $M^{\prime}$, est symétrique de $M^{\prime}$ par rapport à $l$ 'axe $: d x^{\prime}{ }_{l}=d x^{\prime}, y_{1}^{\prime}=-y^{\prime}$. Ceci implique que dx' est forcément une fonction paire de $y, d$ 'où $a=0$.

La condition à satisfaire alors pour qu'il y ait stigmatisme approché dans le plan de front de A est la relation d'Abbe : on dira d'un système qui la vérifie qu'il est aplanétique.

Les conditions d'aplanétisme d'un système optique sont donc

(a) le système optique est rigoureusement stigmatique pour un couple de points $A$ et $A^{\prime}$;

(b) la condition d'Abbe est vérifiee pour tout rayon issu de $\mathrm{A}$.

Un système aplanétique permettra d'obtenir de bonnes images d'objets de petite dimension orthogonaux à l'axe, même pour des rayons fortement inclinés par rapport à l'axe.

Cette condition d'aplanétisme ou condition des sinus d'Abbe sera très utile lors de l'étude des aberrations de systèmes optiques. En termes d'aberrations, les 2 conditions ci-dessus s'expriment sous la forme suivante :

(a) le système optique est corrigé de l'aberration sphérique pour A et $\mathrm{A}$ ' (seule aberration présente pour un objet sur l'axe) ;

(b) le système optique est corrigé de la coma.

Le fait que pour un point objet $\mathrm{B}$ de petite dimension y dans le plan de $\mathrm{A}$, le décalage $d x^{\prime}$ varie comme $y^{2}$ est l'aberration de courbure de champ.

Voyons si ces conditions d'aplanétisme sont remplies dans le cas des systèmes simples dont on a vu au paragraphe précédent qu'ils étaient rigoureusement stigmatiques pour des points à distance finie (dioptre sphérique, miroirs elliptique et hyperbolique). Le système étant constitué d'une surface unique, réfractante ou réfléchissante, on définit les rayons par la position du point d'impact I sur la surface.

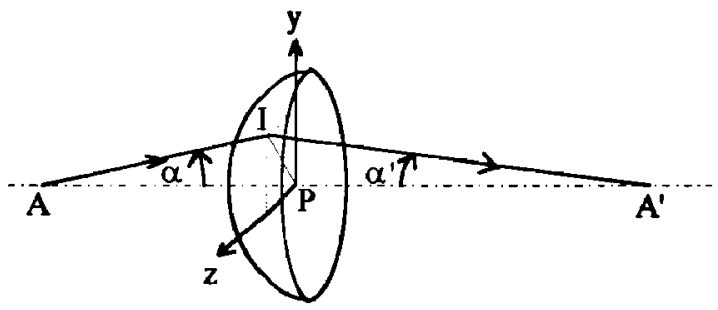

P étant la projection du point I sur l'axe du système, on peut écrire :

$$
\sin \alpha=\frac{\overline{\mathrm{PI}}}{\overline{\mathrm{AI}}} \quad \sin \alpha^{\prime}=\frac{\overline{\mathrm{PI}}}{\overline{\mathrm{A}^{\prime} \mathrm{I}}}
$$

La relation d'Abbe peut s'écrire sous la forme : 


$$
\frac{\sin \alpha^{\prime}}{\sin \alpha}=\frac{m y}{n^{\prime} y^{\prime}}=\operatorname{cste}=\frac{\overline{\mathrm{AI}}}{\mathrm{A}^{\prime} \mathrm{I}}
$$

Ceci n'est satisfait que si I est sur une sphère.

En conclusion, le dioptre sphérique est bien aplanétique pour son centre et pour les points d'Young-Weierstrass, ainsi que le miroir sphérique pour son centre de courbure. Par contre les miroirs elliptique et hyperbolique ne le sont pas.

\subsubsection{Condition d'Herschel}

On choisit maintenant un point $\mathbf{M}$ au voisinage de A sur l'axe $(y=0)$. Par symétrie, son image est forcément sur l'axe, et la relation d'Abbe est automatiquement vérifiée. Le stigmatisme approché sera donc réalisé si la condition d'Herschel est vérifiée pour tout rayon d'angle $\alpha$ issu de A.

Un système rigoureusement stigmatique pour un couple de points $A$ et $A^{\prime}$ et vérifiant la condition d'Herschel permettra d'obtenir de bonnes images d'objets ponctuels même s'ils se déplacent légèrement par rapport à la position idéale le long de l'axe, et pour une grande ouverture des rayons incidents.

\subsubsection{Cas des objets et images à l'infini}

Lorsque l'objet, par exemple, est très éloigné, les paramètres commodes ne sont plus $y$ et $\alpha$, mais l'angle $\theta$ sous lequel est vu l'objet $\mathrm{AB}$ et la hauteur $\mathrm{h}$ du rayon issu de $\mathrm{A}$. On peut relier ces paramètres d'après la figure suivante :

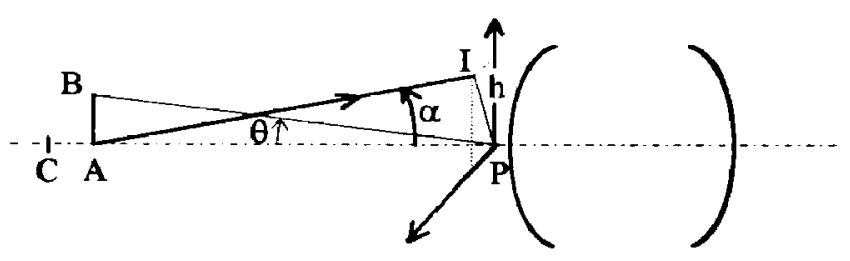

Les angles $\alpha$ et $\theta$ sont petits puisque AP tend vers l'infini :

$$
\alpha=\frac{h}{\mathrm{AP}} \quad \theta=-\frac{y}{\mathrm{AP}}
$$

La condition d'Abbe s'écrit alors :

$$
n^{\prime} y^{\prime} \sin \alpha^{\prime}=-n h \theta
$$

On peut de la même façon écrire la condition d'Abbe pour l'image à l'infini en remplaçant $n^{\prime} y^{\prime} \sin \alpha^{\prime}$ par $-n^{\prime} h^{\prime} \theta^{\prime}$. 
Pour la condition d'Herschel, on caractérisera la position du point éloigné sur l'axe par sa proximitė $\xi_{A}=1 / \mathrm{PA}$ pour le point $\mathrm{A}$, et $\xi_{\mathrm{C}}=1 / \mathrm{PC}$ pour le point $\mathrm{C}$ proche de $\mathrm{A}$ sur l'axe. On peut relier $d \xi=\xi_{C}-\xi_{A}$ à $d x=A C$, sachant que $C$ et $A$ sont proches :

$$
d x=-\frac{d \xi}{\xi_{\mathrm{A}}^{2}}
$$

De plus on a $\alpha=-h \xi_{A}$, ce qui permet de récrire la condition d'Herschel sous la forme :

$$
n^{\prime} d x^{\prime} \sin ^{2} \frac{\alpha^{\prime}}{2}=-\frac{1}{4} n h^{2} d \xi
$$

Voyons ce qu'implique la condition d'aplanétisme pour une surface simple (miroir ou dioptre). En particulier, on voudrait voir si le miroir parabolique, stigmatique pour le point objet à l'infini sur l'axe, est aplanétique. Pour un système formé d'une surface unique, les rayons incident et émergent se coupent en un point I de la surface :

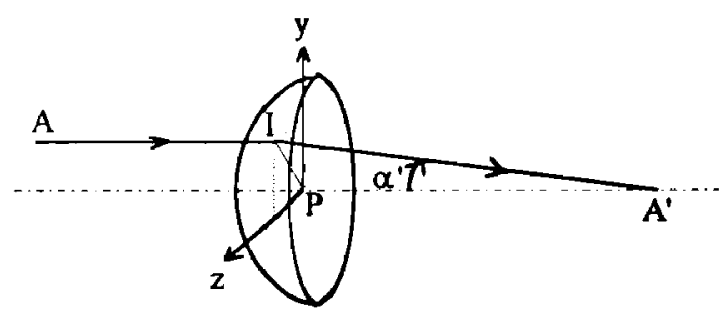

On peut écrire la condition d'Abbe pour un objet à l'infini sous la forme :

$$
\begin{gathered}
\frac{h}{\sin \alpha^{\prime}}=-\frac{n^{\prime} y^{\prime}}{n \theta}=c s t e \\
\text { ici : } \frac{\overline{\mathrm{PI}}}{\sin \alpha^{\prime}}=\text { cste }=\overline{\mathrm{A}^{\prime} \mathrm{I}}
\end{gathered}
$$

La condition d'Abbe implique donc que le point I décrive la surface d'une sphère de centre A' lorsque le rayon issu de A varie. Le miroir parabolique n'est donc pas aplanétique, et tous les dioptres non sphériques non plus.

\section{Conclusion :}

Dans cette partie, on a vu qu'il existait un certain nombre de systèmes optiques rigoureusement stigmatiques : miroir plan pour tous les points, miroir sphérique pour son centre de courbure, miroirs elliptique et hyperbolique pour les foyers des coniques, miroir parabolique pour le point 
à l'infini sur l'axe, dioptre sphérique pour son centre de courbure et les points d'YoungWeierstrass. Parmi tous ces systèrnes, seuls ceux dont la surface est sphérique ou plane sont aplanétiques.

Il existe bien sûr dautres systèmes optiques plus complexes qui sont stigmatiques même pour des angles d'incidence grands (télescope de Ritchey-Chrétien, objectifs d'appareil photo grand angle). Il s'agit alors d'associer des systèmes dont les aberrations se compensent (on a vu par exemple que l'aberration sur l'axe d'un miroir sphérique concave était de signe opposè à celle d'un dioptre plan), et ce en fonction du type d'images que l'on veut faire (objet de grande dimension et système peu ouvert, ou objet de petite dimension avec un système optique très ouvert, etc).

\section{APPROXIMATION LINÉAIRE OU APPROXIMATION DE GAUSS}

Nous avons vu dans la partie précédente que les conditions de stigmatisme rigoureux ne sont remplies que pour un nombre limité de systèmes optiques, et pour des couples de points très particuliers. Une première forme de stigmatisme approché consistait à n'autoriser que des petits déplacements au voisinage des points stigmatiques, tout en gardant des rayons très inclinés sur l'axe : c'est le stigmatisme approché du type Abbe ou Herschel.

Pour toutes les situations où ces conditions de stigmatisme ne sont pas remplies, l'image va présenter des aberrations. Cependant, lorsqu'on diaphragme ces systèmes optiques et qu'on observe des objets pas trop grands, on constate que les images redeviennent de bonne qualité.

Nous commencerons cette partie en traitant le cas du dioptre sphérique : l'approximation des angles faibles conduira à une relation linéaire entre les paramètres définissant le rayon incident et ceux définissant le rayon émergent et à une relation de conjugaison objet/image indépendante du rayon choisi.

Nous généraliserons cette propriété à tout système optique homogène centré, lorsqu'on se limite à des rayons faiblement inclinés sur l'axe et à des objets de petite dimension. Le stigmatisme est alors réalisé pour tous les points objets. C'est le cadre de l'approximation linéaire de l'optique géométrique ou approximation de Gauss. On appelle encore ce domaine d'étude l'optique paraxiale et les rayons sont appelés les rayons paraxiaux.

Nous verrons ensuite comment les coefficients de ces relations linéaires sont reliés aux caractéristiques du système optique et à celles des objets et images. Ceci permettra de définir un certain nombre de points cardinaux caractéristiques d'un système optique centré, à partir desquels on pourra calculer ou construire n'importe quelle image

\subsection{Exemple d'approximation linéaire dans le cas du dioptre sphérique}

\subsection{Calcul rigoureux}

On peut grâce à la loi vectorielle de Snell-Descartes de la réfraction calculer le rayon émergent à partir du rayon incident sans aucune approximation. Pour cela il faut:

- calculer l'intersection I du rayon incident avec la surface du dioptre ;

- calculer la normale $\mathbf{N}$ au dioptre en I (vecteur unitaire porté par $\mathbf{C I}$ ) ;

- calculer l'angle $\mathrm{i}$ entre $\mathbf{u}$ et $\mathbf{N}$;

- calculer l'angle i' réfracté en utilisant la loi de réfraction $n \sin i=n^{\prime} \sin \mathrm{i}^{\prime}$;

-calculer $\mathbf{u}^{\prime}$ en utilisant la loi vectorielle $n \mathbf{u}-n^{\prime} \mathbf{u}^{\prime}=\left(n \cos i-n^{\prime} \cos i^{\prime}\right) \mathbf{N}$.

Le rayon émergent est finalement défini par I et u'. 
Pour déterminer l'image d'un point avec cette méthode, il va falloir répéter ce calcul pour un grand nombre de rayons issus du point objet, et chercher lintersection des rayons émergents avec un plan orthogonal à l'axe du système pour trouver la position de l'image. Il s'agit là d'un calcul long et fastidieux et pour lequel il serait déjà très utile de connaître au moins approximativement la position de l'image. Pour cela nous allons nous intéresser aux comportements des rayons qui restent proches de l'axe.

\subsubsection{Approximation linéaire}

On se place dans le repère $S x y z$, où $S$ est un point du dioptre sphérique et $S x$ l'axe passant par $C$ (axe optique du dioptre).

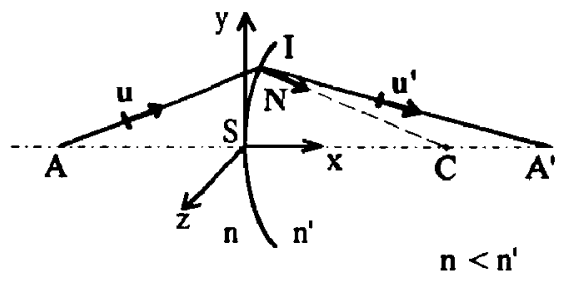

Puisqu'on se limite aux rayons proches de l'axe, on va pouvoir écrire :

- la loi de la réfraction sous la forme $n i=n^{\prime} i^{\prime}$ et $n^{\prime} \mathrm{u}^{\prime}-n \mathrm{u}=\left(n^{\prime}-n\right) \mathbf{N}$;

- les coordonnées des vecteurs $\mathbf{u}, \mathbf{u}^{\prime}$ et $\mathbf{N}$ sous la forme :

$$
\mathbf{u}\left[\begin{array}{l}
1 \\
\beta \\
\gamma
\end{array}\right] \quad \mathbf{u}^{\prime}\left[\begin{array}{c}
1 \\
\beta^{+} \\
\gamma^{*}
\end{array}\right] \quad \mathbf{N}\left[\begin{array}{c}
1 \\
-\frac{y_{1}}{R} \\
-\frac{z_{I}}{R}
\end{array}\right]
$$

où $\mathrm{y}_{1}$ et $\mathrm{z}_{\mathrm{I}}$ sont les coordonnées du point I et $\mathrm{R}=\overline{\mathrm{SC}}$ est le rayon de courbure (algébrique) du dioptre. L'approximation conduit notamment à considérer que le point I est dans le plan Syz tangent au dioptre $\left(\mathrm{x}_{\mathrm{I}}=0\right)$.

On obtient deux relations du même type que l'on peut regrouper en définissant de nouveaux paramètres complexes :

$$
\begin{aligned}
Z=y_{\mathrm{I}}+j z_{\mathrm{I}} \quad \Omega & =n \beta+j n \gamma \quad \Omega^{\prime}=n^{\prime} \boldsymbol{\beta}^{\prime}+j n^{\prime} \gamma^{\prime} \\
\Omega^{\prime} & =\Omega+\left(n-n^{\prime}\right) \frac{Z}{R}
\end{aligned}
$$

$(Z, \Omega)$ définit le rayon incident et $\left(Z, \Omega^{\prime}\right)$ le rayon émergent. On obtient finalement une relation linéaire entre ces paramètres que l'on peut écrire sous forme matricielle :

$$
\left[\begin{array}{l}
\mathrm{Z} \\
\Omega
\end{array}\right]=\left[\begin{array}{cc}
1 & 0 \\
-\frac{n^{\prime}-n}{R} & 1
\end{array}\right]\left[\begin{array}{l}
\mathrm{Z} \\
\Omega
\end{array}\right]
$$


Notons que le fait d'inclure les indices dans les définitions des paramètres $\Omega$ conduit à une matrice de déterminant $\mathbf{I}$. Pour un dioptre plan comme pour un miroir plan $(R=\infty)$, la matrice est l'identité.

\subsubsection{Calcul de l'image d'un point objet sur l'axe}

L'objet est le point A sur l'axe, d'où part le rayon dirigé par u. On cherche la position de A', intersection du rayon émergent avec l'axe. Puisque le dioptre est de révolution, on peut pour simplifier prendre un point I dans le plan Sxy, d'où $z_{I}=0$ et $y=\gamma^{\prime}=0$. De plus les hypothèses précédentes conduisent à prendre I dans le plan tangent Syz. Finalement on peut déterminer $\mathrm{A}^{\prime}$ à partir d'une figure où le dioptre est schématisé par son plan tangent :

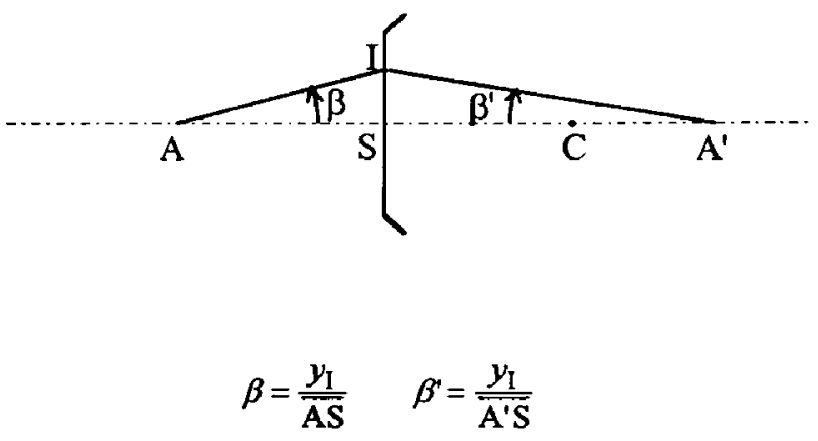

La relation linéaire précédente entre les rayons conduit à :

$$
\frac{n^{\prime}}{\overline{\mathrm{A}^{\prime} \mathrm{S}}}=\frac{n}{\overline{\mathrm{AS}}}+\frac{\left(n-n^{\prime}\right)}{R}
$$

La dépendance en $y_{I}$ a disparu. La position de limage $A^{\prime}$ ne dépend plus du rayon choisi : il y a stigmatisme.

\subsubsection{Validité de l'approximation}

Cherchons les conditions sur les rayons pour que l'approximation de Gauss soit vérifiée. On a vu que les angles des rayons avec la normale au dioptre ou avec l'axe doivent être suffisamment faibles pour que sini $\approx \operatorname{tani} \approx \mathrm{i}$ et $\operatorname{cosi} \approx 1$ : ceci est vérifié à $10 \%$ près pour des angles inférieurs à $25^{\circ}$ environ et à $1 \%$ près pour des angles inférieurs à $8^{\circ}$. Il est courant de traduire ces chiffres en termes de nombre d'ouverture du système optique $N=1 / 2 n \sin \alpha$, qui vaut environ 3.5 pour un angle $\alpha$ de $8^{\circ}$ et un milieu d'indice $n=1$; on parle alors d'un système ouvert à $\mathbf{f} / 3.5$. L'approximation de Gauss sera donc très proche de la réalité pour les systèmes ouverts à $\mathrm{f} / \mathrm{N}$ avec $\mathrm{N}$ supérieur à 4 . 


\subsection{Approximation linéaire et stigmatisme}

Nous allons montrer maintenant dans le cas général les propriétés que nous venons de voir dans le cas particulier du dioptre sphérique.

\subsection{Relation entre rayon incident et rayon émergent}

Dans toute la suite, nous ne considérerons que des systèmes optiques centrés, de révolution autour de leur axe optique $O x$, formés de milieux homogènes séparés par des dioptres : les rayons se présenteront done sous la forme de sections rectilignes changeant de direction aux surfaces. Les rayons incidents et émergents du système seront donc des segments de droite, que l'on pourra caractériser par 4 paramètres.

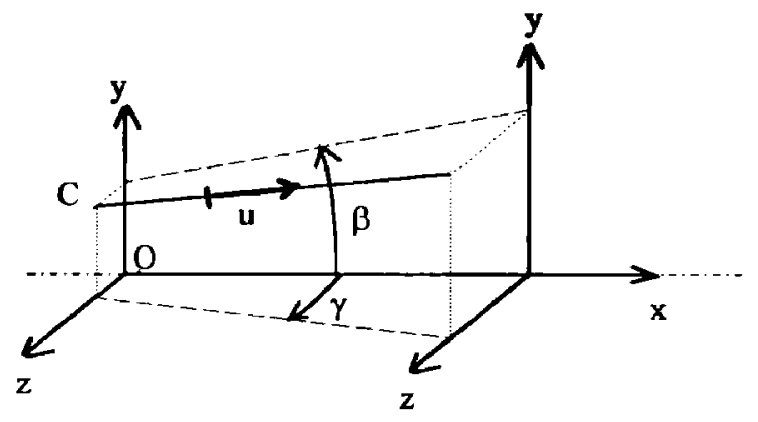

Nous définirons un rayon par son point d'intersection $C\left(y_{1}, z_{1}\right)$ avec le plan $O y z$, et par son vecteur directeur $\mathbf{u}(\beta, \gamma)$, dont les composantes dans le repère $O x y z$ s'écrivent:

$$
C\left[\begin{array}{c}
0 \\
y_{1} \\
z_{1}
\end{array}\right] \quad u=\frac{1}{1+\operatorname{tg}^{2} \beta+\operatorname{tg}^{2} \gamma}\left[\begin{array}{c}
1 \\
\operatorname{tg} \beta \\
\operatorname{tg} \gamma
\end{array}\right]
$$

Nous inclurons également l'information concernant l'indice du milieu dans lequel se propage ce rayon en prenant comme paramètres $n \beta$ et $m \gamma$, au lieu de $\beta$ et $\gamma$.

De façon complètement générale, chaque paramètre du rayon émergent $\left(y_{1}^{\prime}, z_{1}^{\prime}, n^{\prime} \beta^{\prime}, n^{\prime} \gamma^{\prime}\right)$ peut s'écrire comme une série de Taylor en fonction des paramètres $\left(y_{1}, z_{1}, n \beta, m \gamma\right)$ du rayon incident, du genre

$$
y_{1}^{\prime}=a_{0}+a_{1} y_{1}+a_{2} z_{1}+a_{3} n \beta+a_{4} n \gamma+\text { termes d'ordres supérieurs }
$$

De plus, le système étant de révolution autour de l'axe $O x$, le rayon suivant l'axe $O x$ de paramètres $(0,0,0,0)$ sort du système optique toujours suivant l'axe, donc $a_{0}=0$.

L'approximation linéaire va consister à négliger les termes d'ordre supérieur ou égal à 2 . Ceci revient à dire que les rayons considérés sont proches de l'axe du système. En particulier les angles $\beta$ et $\gamma$ sont faibles, de sorte que le vecteur directeur $\mathbf{u}$ du rayon a pour coordonnées : 


$$
\mathbf{u}\left[\begin{array}{l}
1 \\
\beta \\
\gamma
\end{array}\right]
$$

La relation entre les paramètres du rayon émergent et ceux du rayon incident peut alors s'écrire sous forme matricielle :

$$
\left[\begin{array}{c}
y_{1}^{\prime} \\
z_{1}^{\prime} \\
n^{\prime} \beta^{\prime} \\
n^{\prime} \gamma^{\prime}
\end{array}\right]=\mathbf{M}\left[\begin{array}{c}
y_{1} \\
z_{1} \\
n \beta \\
m \gamma
\end{array}\right]
$$

M est une matrice $4 \times 4$, à coefficients réels $a_{i j}$, qui dépend du système optique.

\subsubsection{Simplification liée à la symétrie de révolution du système}

Ceci permet de simplifier beaucoup la matrice $4 \times 4$ précédente. On peut montrer que beaucoup de termes de la matrice sont nuls ou égaux en utilisant par exemple les propriétés suivantes :

a) un rayon incident contenu dans le plan Oxy donne un rayon émergent contenu dans le plan Oxy (car Oxy contient l'axe de symétrie) : pour $z_{1}=\gamma=0$, on a toujours $z_{1}^{\prime}=\gamma^{\prime}=$ 0 ; donc les coefficients $\mathbf{a}_{21}, \mathbf{a}_{23}, \mathbf{a}_{41}, \mathbf{a}_{43}$ de la matrice sont nuls.

b) même chose pour un rayon incident dans le plan $O x z: a_{12}, a_{14}, a_{32}, a_{34}$ sont nuls.

c) Le système est invariant par une rotation de $90^{\circ}$ autour de $\mathrm{Ox}$.

On choisit un rayon incident $\mathrm{R}_{1}\left(y_{1}, 0, n \beta, 0\right)$, contenu dans le plan $O x y$, qui donne un rayon $R_{1}^{\prime}\left(y_{1}^{\prime}, 0, n^{\prime} \beta^{\prime}, 0\right)$, contenu dans le plan $O x y$. Le rayon $R_{2}\left(0, y_{1}, 0, n \beta\right)$, qui se déduit de $R_{1}$ par une rotation de $90^{\circ}$, donnera un rayon émergent $\mathrm{R}_{2}^{\prime}\left(0, y_{1}^{\prime}, 0, n^{\prime} \beta^{\prime}\right)$, qui se déduit de $R_{2}^{\prime}$ par une même rotation de $90^{\circ}$. Il y a donc égalité 2 à 2 des coefficients : $a_{11}=a_{22}, a_{13}=a_{24}, a_{31}=a_{42}, a_{33}=a_{44}$

Finalement la matrice $\mathbf{M}$ se récrit sous la forme :

$$
\mathbf{M}=\left[\begin{array}{cccc}
a_{I} & 0 & a_{2} & 0 \\
0 & a_{I} & 0 & a_{2} \\
a_{3} & 0 & a_{4} & 0 \\
0 & a_{3} & 0 & a_{4}
\end{array}\right]
$$

On peut simplifier l'écriture de la relation linéaire en passant en paramètres complexes :

$$
\begin{gathered}
Z=y_{1}+j z_{1} \quad \Omega=n \beta+j n \gamma \\
{\left[\begin{array}{l}
Z^{\prime} \\
\Omega
\end{array}\right]=\left[\begin{array}{ll}
a_{1} & a_{2} \\
a_{3} & a_{4}
\end{array}\right]\left[\begin{array}{l}
Z \\
\Omega
\end{array}\right]}
\end{gathered}
$$


La transformation entre les rayons est donc caractérisée par une matrice $2 \times 2$ à coefficients réels.

Note : la symétrie de révolution du système implique notamment que la relation rayon incident - rayon émergent est invariante par symétrie par rapport à l'axe. Si on change de signe tous les paramètres du rayon incident, tous les paramètres du rayon émergent doivent changer de signe. Ceci implique que dans le développement des $\left(y_{l}^{\prime}, z_{1}^{\prime}, n^{\prime} \beta^{\prime}, n^{\prime} \gamma^{\prime}\right)$ en fonction des $\left(y_{1}, z_{l}, n \beta, n \gamma\right)$, il n'y a pas de termes pairs. Le premier ordre supérieur à l'approximation linéaire est donc un terme du 3ème ordre en fonction des paramètres des rayons.

\subsubsection{Stigmatisme pour un point objet sur l'axe}

Nous allons nous intéresser maintenant à ce qu'implique l'approximation précédente pour la formation des images.

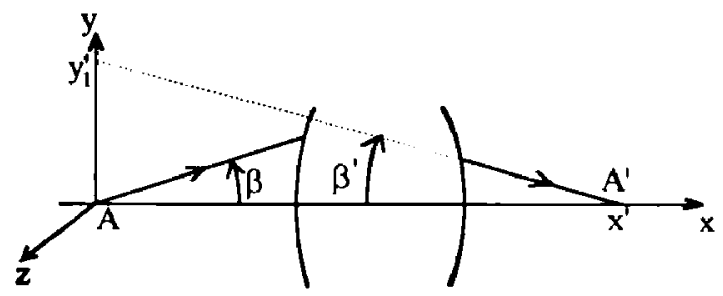

Pour déterminer l'image d'un point objet $\mathrm{A}$ sur l'axe, on va considérer un rayon quelconque issu de ce point et chercher l'intersection du rayon émergent avec l'axe. Choisissons comme repère $A x y z$, où Ax est l'axe du système et Axy contient le rayon incident. Les coordonnées d'un rayon issu de $A$ sont $Z=0$ et $\Omega=n \beta$. Le rayon émergent correspondant a pour coordonnées $Z^{\prime}=a_{2} n \beta=y_{1}^{\prime}$ et $\Omega^{\prime}=a_{4} n \beta=n^{\prime} \beta^{\prime}$. La position de l'intersection $A^{\prime}$ avec l'axe est :

$$
x^{\prime}=\overline{\mathrm{AA}^{\prime}}=-\frac{y_{1}^{\prime}}{\beta^{\prime}}=-\frac{a_{2} n^{\prime}}{a_{4}}
$$

Elle est donc indépendante du rayon choisi dans le plan Oxy. Par symétrie de révolution, la position de l'image est indépendante du rayon choisi quel qu'il soit (par contre elle dépend bien de A via les coefficients $a_{2}$ et $a_{4}$ de la matrice exprimée dans le repère lié à A).

\subsubsection{Effet d'un changement de référentiel}

Considérons l'expression $\left(Z_{1}, \Omega_{1}\right)$ d'un rayon dans un repère $O_{1} x y z$ et cherchons comment elle est modifiée si on passe à un repère $\mathrm{O}_{2} x y z$, où l'origine est translatée de $\mathrm{O}_{1} \mathrm{O}_{2}$ le long de $\mathrm{O}_{1} \mathrm{x}$. On montre facilement que les nouvelles coordonnées $\left(Z_{2}, \Omega_{2}\right)$ s'écrivent :

$$
\left[\begin{array}{l}
Z_{2} \\
\Omega_{2}
\end{array}\right]=\left[\begin{array}{ll}
1 & \overline{0_{1} 0_{2}} \\
0 & 1
\end{array}\right]\left[\begin{array}{l}
Z_{1} \\
\Omega_{1}
\end{array}\right]
$$


On appellera matrice de déplacement la matrice de translation du repère. On pourra donc choisir le repère le plus pratique pour écrire les coordonnées d'un rayon ou la matrice d'un système, puis multiplier par des matrices de déplacement pour changer de repère. Notons que le déterminant de cette matrice vaut 1 , de sorte que le déterminant de la matrice ne changera pas avec le repère.

Par exemple on aura intérêt à écrire les coordonnées $(Z, \Omega)$ du rayon incident dans un repère dont l'origine est l'objet $A$ et le rayon émergent $\left(Z^{\prime}, \Omega^{\prime}\right)$ dans le repère centré en $A^{\prime}$ image de A. On a alors:

$$
\left[\begin{array}{l}
Z^{\prime} \\
\Omega^{\prime}
\end{array}\right]_{\left(A^{\prime} x y z\right)}=\left[\begin{array}{cc}
1 & \frac{x^{\prime}}{n^{\prime}} \\
0 & 1
\end{array}\right]\left[\begin{array}{ll}
a_{1} & a_{2} \\
a_{3} & a_{4}
\end{array}\right]\left[\begin{array}{l}
Z \\
\Omega
\end{array}\right]_{(A x y z)}
$$

Comme $x^{\prime}=-n^{\prime} a_{2} / a_{4}$, la matrice de transformation s'écrit sous la forme :

$$
\left[\begin{array}{l}
Z^{\prime} \\
\Omega
\end{array}\right]_{\left(A^{\prime} x y z\right)}=\left[\begin{array}{cc}
a_{1}^{\prime} & 0 \\
a_{3}^{\prime} & a_{4}^{\prime}
\end{array}\right]\left[\begin{array}{l}
\mathrm{Z} \\
\Omega
\end{array}\right]_{A(x y z)}
$$

La matrice d'un système optique entre deux points conjugués est donc simplifiée.

\subsubsection{Stigmatisme pour un point objet.voisin de l'axe}

Considérons maintenant un point objet B, contenu dans le plan Ayz et voisin de A. Un rayon quelconque issu de $\mathrm{B}(0, y, z)$ aura pour paramètres dans le repère $\mathrm{Axyz}: Z=y+j z$ et $\Omega=n \beta$ $+j r \gamma$. Le fait que B soit voisin de l'axe est nécessaire pour qu'on puisse appliquer l'approximation linéaire avec $y$ et $z$ petits.

Remarque : dans ce cas le rayon incident $(B, u) n$ 'est pas dans un plan contenant l'axe ; on ne peut donc rien dire de particulier sur le rayon émergent.

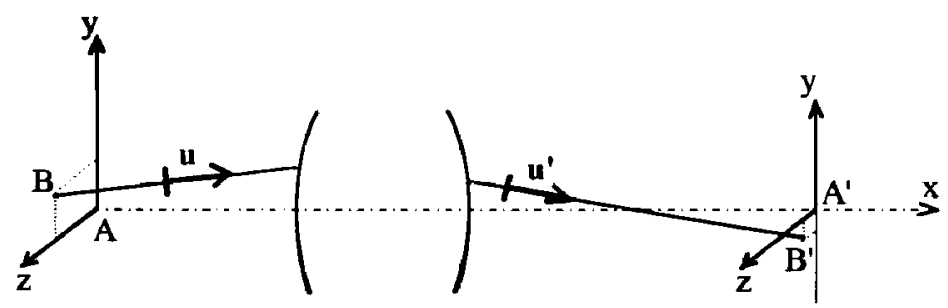

Pour déterminer le rayon émergent, on utilise la matrice du système entre les points $\mathrm{A}$ et $A^{\prime}$ : on voit alors immédiatement apparaître, du fait de $a_{2}^{\prime}=0$, le fait que le paramètre $Z^{\prime}$ du rayon émergent ne dépend pas de $\Omega$, donc de la direction du rayon incident. Or $Z^{\prime}$ représente justement les coordonnées du point d'intersection du rayon émergent avec le plan $A^{\prime} y z$ : on trouve donc que tous les rayons incidents issus de $B$ se coupent en un même point B' situé dans le plan de A'. Il y a donc stigmatisme, et l'image d'un plan orthogonal à l'axe est un plan 
orthogonal à l'axe. De plus si B est suivant $A y(z=0)$, son image est suivant l'axe $A^{\prime} y^{\prime}$, autrement dit $A, A^{\prime}, B$ et $B^{\prime}$ sont dans un même plan.

Finalement, nous avons montré que pour un système optique centré et des rayons satisfaisant l'approximation linéaire (peu inclinés et peu distants de l'axe), il y a stigmatisme quel que soit le point objet sur l'axe ou voisin de l'axe, et que l'image d'une portion de plan voisine de l'axe est une portion de plan.

\subsubsection{Autres applications de ce formalisme}

Le formalisme matriciel que nous avons développé permet de bien mettre en évidence le caractère linéaire de l'approximation de Gauss.

Il est également bien adapté au calcul informatique, qui devient utile dans le domaine paraxial lorsque le nombre de dioptres devient très grand. On peut même étendre ce type de formalisme matriciel pour inclure également les ordres supérieurs de calcul (matrices plus grandes).

On retrouvera également ces matrices $2 \times 2$ lors de l'étude des cavités lasers, où elles permettent de déterminer les conditions de stabilité et les paramètres des faisceaux émis dans le mode fondamental (faisceaux gaussiens).

Enfin, ce type de formalisme se retrouve pour l'optique des faisceaux d'électrons, ou pour tout type de faisceaux de particules pour lequel on peut faire une approximation linéaire.

Il est toutefois déconseillé d'utiliser les matrices pour des systèmes simples pour lesquels l'utilisation des constructions graphiques et des formules de conjugaison, que nous verrons par la suite, est beaucoup plus rapide et permet de visualiser la cohérence des résultats.

\subsection{Caractérisation des systèmes optiques dans le cadre de l'approximation de Gauss}

Dans ce paragraphe, nous allons relier les matrices, en particulier leurs coefficients, aux propriétés du système optique qu'elles représentent ainsi qu'à la relation entre la position de l'objet et celle de l'image (relation de conjugaison).

Suite à la remarque du \$3.2.4., nous choisirons les repères Axyz pour le rayon incident et A'xyz pour le rayon émergent, de sorte que le coefficient $a_{2}^{\prime}$ de la matrice du système optique soit nul. Pour simplifier l'écriture, nous reprendrons les notations sans prime $a_{1}, a_{2}, a_{3}, a_{4}$ pour les coefficients de la matrice, avec donc $a_{2}=0$.

\subsubsection{Grandissements. Convergence.}

*Considérons un rayon issu de $\mathrm{A}$ et incliné d'un angle $\alpha$ sur l'axe. Prenons comme plan Axy le plan contenant ce rayon.
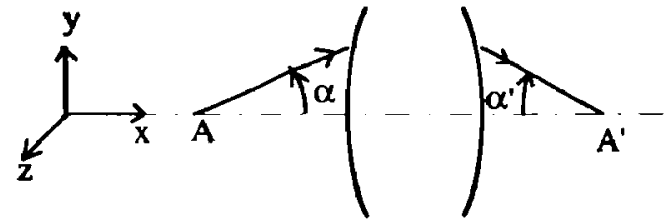
La relation entre rayons s'écrit :

$$
\left[\begin{array}{c}
0 \\
n^{\prime} \alpha^{\prime}
\end{array}\right]=\left[\begin{array}{cc}
a_{1} & 0 \\
a_{3} & a_{4}
\end{array}\right]\left[\begin{array}{c}
0 \\
n \alpha
\end{array}\right]
$$

Le coefficient $a_{4}$ de la matrice est donc relié au grandissement angulaire $\mathrm{g}_{\alpha}=\alpha^{\prime} / \alpha$ par :

$$
a_{4}=\frac{n^{\prime}}{n} g_{\alpha}
$$

*Considérons un point $\mathrm{B}$ voisin de A de coordonnée y. La relation de transformation d'un rayon quelconque issu de $B$ s'écrit :

$$
\left[\frac{\mathrm{y}^{\prime}}{\Omega^{\prime}}\right]=\left[\begin{array}{ll}
a_{1} & 0 \\
a_{3} & a_{4}
\end{array}\right]\left[\begin{array}{l}
\mathrm{y} \\
\Omega
\end{array}\right]
$$

Le coefficient $a_{1}$ est donc égal au grandissement transversal $\mathrm{g}_{\mathrm{y}}=\mathrm{y}^{\prime} / \mathrm{y}$.

${ }^{*}$ Considérons un autre couple de points quelconques $A_{1}$ et $A_{1}^{\prime}$ (pas forcément conjugués) et écrivons la nouvelle forme de la matrice du système. On peut l'obtenir en utilisant les matrices de déplacement définies au $\$ 3.2 .4$ :

$$
\left[\begin{array}{l}
Z_{1}^{\prime} \\
\Omega_{1}^{\prime}
\end{array}\right]_{\left(A^{\prime}, x y z\right)}=\left[\begin{array}{cc}
1 & \overline{\mathrm{A}^{\prime} \mathrm{A}_{1}^{\prime}} \\
0 & 1
\end{array}\right]\left[\begin{array}{cc}
n_{1}^{\prime} & 0 \\
a_{3} & a_{4}
\end{array}\right]\left[\begin{array}{cc}
1 & \overline{\mathrm{A}_{1} \mathrm{~A}} \\
0 & 1
\end{array}\right]\left[\begin{array}{l}
\mathrm{Z}_{1} \\
\Omega_{1}
\end{array}\right]_{\left(\mathrm{A}_{1} x y z\right)}=\left[\begin{array}{ll}
a_{1}^{\prime} & a_{2}^{\prime} \\
a_{3}^{\prime} & a_{4}^{\prime}
\end{array}\right]\left[\begin{array}{l}
\mathrm{Z}_{1} \\
\Omega_{1}
\end{array}\right]_{\left(\mathrm{A}_{1} x y z\right)}
$$

On remarque que le coefficient $a^{\prime}{ }_{3}$ de la nouvelle matrice est égal à $a_{3}$. Il s'agit donc d'un coefficient qui est indépendant des origines choisies pour les repères : c'est une caractéristique du système optique.

On appellera convergence $C$ du système optique l'opposé de $a_{3}$. Un système optique sera dit convergent si $\mathrm{C}>0$ : un tel système ramène les rayons vers l'axe. Par exemple un dioptre

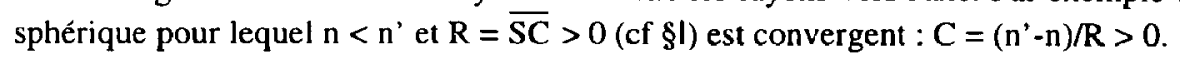

Nous appellerons systèmes focaux ceux pour lesquels $C \neq 0$ et systèmes afocaux ceux pour lesquels $\mathrm{C}=0$.

\subsubsection{Formules de conjugaison et relations entre les grandissements}

Reprenons la relation matricielle de changement de repères $\left(A, A^{\prime}\right)$ vers $\left(A_{1}, A^{\prime}\right)$ écrite plus haut, mais en considérant maintenant le cas où $A_{1}$ et $A^{\prime}$, sont conjugués. On va chercher à déterminer les nouveaux coefficients $a_{2}^{\prime}, a_{3}{ }_{3}$ et $a_{4}{ }_{4}$.

${ }^{*}$ On sait puisque $\mathrm{A}_{1}$ et $\mathrm{A}^{\prime}$, sont conjugués que le terme $a_{2}{ }_{2}$ doit être nul. On obtient donc une relation entre les positions de $A, A^{\prime}, A_{1}$ et $A^{\prime}{ }_{1}$ que l'on peut mettre sous la forme :

$$
\frac{\left(g_{y}\right)_{\mathrm{AA}^{\prime}}}{\overline{\mathrm{A}^{\prime} \mathrm{A}_{1}^{\prime}}}-\frac{\left(g_{\mathrm{a}}\right)_{\mathrm{AA}^{\mathrm{A}}}}{\overline{\mathrm{AA}_{1}}}=\frac{C}{n^{\prime}}
$$

Si l'on connaît la position d'un couple de points conjugués particuliers et les grandissements pour ce couple de points, on peut ainsi déterminer la position de tous les 
autres couples de points conjugués. On appellera cette relation la formule de conjugaison du système avec origine aux points $\left(\mathrm{A}, \mathrm{A}^{\prime}\right)$, ou encore formule de conjugaison de Descartes. On retrouve par exemple la relation de conjugaison du dioptre sphérique démontrée au $\$ 3.1 .2$ avec $\mathrm{A}=\mathrm{A}^{\prime}=\mathrm{S}$.

Remarque :Cette relation ne conceme a priori que les points objets sur l'axe. En fait, on a démontré au $\$ 3.2 .5$ que l'image d'un point objet $B$ dans le plan de $A$ se trouve dans le plan de $A^{\prime}$ : la formule de conjugaison donne la projection sur l'axe de l'image $B^{\prime}$ et il suffit de multiplier la distance $\overline{\mathrm{AB}}$ par le grandissement transversal (coefficient $a_{l}$ de la matrice) pour trouver $\overline{\mathrm{A}^{\prime} \mathrm{B}}$.

*On peut mettre la formule de conjugaison ci-dessus sous la forme :

$$
\frac{\overline{\mathrm{AA}_{1}}}{\overline{\mathrm{A}^{\prime} \mathrm{A}_{1}^{\prime}}}=\frac{g_{\alpha}}{g_{y}}+\frac{C}{n^{\prime} g_{y}} \overline{\mathrm{AA}_{1}}
$$

En considérant un point $A_{1}$ très proche de $A$, de sorte que le dernier terme de l'égalité soit négligeable, on voit alors apparaître le grandissement axial, rapport entre le petit déplacement $d x^{\prime}=\overline{\mathrm{A}^{\prime} \mathrm{A}_{1}^{\prime}}$ de l'image correspondant au petit déplacement $d x=\overline{\mathrm{AA}_{1}}$ de l'objet :

$$
g_{x}=\frac{\overline{\mathrm{A}^{\prime} \mathrm{A}_{1}^{\prime}}}{\overline{\mathrm{AA}_{1}}}=\frac{d x^{\prime}}{d x}=\frac{g_{y}}{g_{\alpha}}
$$

On obtient donc une relation entre les trois grandissements correspondants à un couple de points conjugués $\left(A, A^{\prime}\right)$ :

$$
g_{y}=g_{x} g_{\alpha}
$$

* Le déterminant de la nouvelle matrice est égal au produit des déterminants des trois matrices. Comme les matrices de déplacements ont pour déterminant 1 , on a donc $a^{\prime} d^{\prime}=a d$. La quantité ad est donc conservée quel que soit le couple de points conjugués. Ceci peut s'écrire :

$$
\frac{n^{\prime}}{n} g_{y} g_{\alpha}=\text { cste }
$$

On peut déterminer cette constante en remarquant que la matrice du dioptre sphérique a pour déterminant 1 (cf $\$ 3.1 .2$ ). Dans l'approximation linéaire, tout système va pouvoir être ramené à une association de dioptres sphériques, donc sa matrice aura également un déterminant de 1 . On obtient donc la relation suivante :

$$
n y \alpha=n^{\prime} y^{\prime} \alpha
$$

qui est appelée relation de Lagrange-Helmholtz. On peut remarquer que c'est l'équivalent de la relation d'Abbe (cf $\$ 2.4$ ) pour des petits angles.

Finalement on voit que deux relations relient entre eux les trois grandissements, transversal, angulaire et axial, de sorte que seule la donnée de l'un d'entre eux est nécessaire.

*A partir des deux relations reliant les grandissements, on peut encore écrire :

$$
g_{x}=\frac{n^{\prime}}{n} g_{y}^{2}
$$


Le grandissement axial est donc toujours positif, ce qui signifie que objet et image se déplacent toujours dans le même sens. D'autre part lorsque l'image est dilatée d'un facteur 2 suivant $y$, elle est dilatée d'un facteur 4 suivant $x$ : les proportions suivant $x$ et $y$ ne sont pas conservées.

${ }^{*}$ Calculons les coefficients $a^{\prime}{ }_{1}$ et $a^{\prime}{ }_{4}$ en fonction de $a_{1}$ et $a_{4}$. Ceci permet de relier les grandissements pour $\left(A_{1}, A^{\prime}\right)$ aux grandissements pour $\left(A, A^{\prime}\right)$ :

$$
\begin{aligned}
& \left(g_{y}\right)_{\mathrm{A}_{1} \mathrm{~A}_{1}^{\prime}}=\left(g_{y}\right)_{\mathrm{AA}^{\prime}}-\frac{C}{n^{\prime}} \overline{\mathrm{A}^{\prime} \mathrm{A}_{1}^{\prime}} \\
& \left(g_{\alpha}\right)_{\mathrm{A}_{1} \mathrm{~A}_{1}^{\prime}}=\left(g_{\alpha}\right)_{\mathrm{AA}^{\prime}}+\frac{C}{n^{\prime}} \overline{\mathrm{AA}_{\mathrm{I}}}
\end{aligned}
$$

L'intérêt de ces relations est de montrer que pour un système focal $(C \neq 0)$, on peut réaliser n'importe quel grandissement transversal ou n'importe quel grandissement angulaire (mais les deux sont liés) à condition de bien choisir le couple de points.

Par contre pour les systèmes afocaux $(\mathrm{C}=0)$, les grandissements sont indépendants du couple de points. La relation de conjugaison écrite plus haut prend alors la forme simple :

$$
\frac{x^{\prime}}{x}=\frac{\overline{\mathrm{A}^{\prime} \mathrm{A}_{1}^{\prime}}}{\overline{\mathrm{AA}_{1}}}=\text { cste }=\frac{g_{y}}{g_{\alpha}}=\frac{n^{\prime}}{n} g_{y}{ }^{2}
$$

et ce, quels que soient les points conjugués choisis pour origines des repères.

\subsubsection{Systèmes focaux}

Dans le cas des systèmes focaux, nous allons pouvoir définir un ensemble de points particuliers appelés points cardinaux, à partir desquels on pourra construire l'image de n'importe quel objet.

\subsubsection{Points cardinaux}

\section{* Points principaux}

Ces points $\mathrm{H}$ et $\mathrm{H}^{\prime}$ sont des points conjugués pour lesquels le grandissement transversal vaut 1. D'après la relation de Lagrange-Helmholtz, le grandissement angulaire vaut alors $n / n$ '.

Nous venons de voir que pour un système focal, on peut toujours trouver de tels points. En prenant ces points comme origine, la matrice a pour coefficient $a_{1}=1$ et toujours $a_{2}=0$ puisque les points sont conjugués. La formule de conjugaison avec origine aux points principaux s'écrit :

$$
\frac{n^{\prime}}{x^{\prime}}-\frac{n}{x}=C \quad \text { avec } x^{\prime}=\overline{\mathrm{H}^{\prime} \mathrm{A}^{\prime}} \text { et } x=\overline{\mathrm{HA}}
$$

Les plans perpendiculaires à l'axe contenant les points principaux sont appelés plans principaux. Ils possèdent une propriété qui sera très utile pour la construction géométrique des rayons. Considérons un rayon incident parallèle à l'axe optique du système. Il coupe le plan 
principal objet en un point I, donc le rayon émergent correspondant doit passer par le point I' image de I par le système.

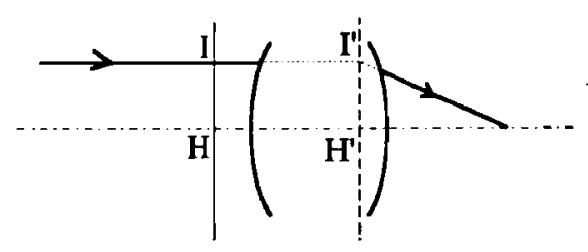

Or l'image de I se trouve très simplement puisqu'on sait d'une part que I' appartient au plan principal image, d'autre part que le grandissement vaut 1 . On obtient donc I' à l'intersection du rayon incident prolonge et du plan principal image.

On peut faire cette construction pour n'importe quel rayon incident parallèle à l'axe : on voit ainsi que le plan principal image est lintersection des rayons incidents parallèles à l'axe et des rayons émergents correspondants.

Un raisonnement analogue permet de montrer que le plan principal objet est lintersection des rayons émergents parallèles à l'axe et des rayons incidents correspondants.

\section{* Foyers}

Le foyer image est l'image d'un point à l'infini sur l'axe. Sa position, repérée par rapport au point principal image $\mathrm{H}^{\prime}$, est obtenue en prenant $\mathrm{A}$ à l'infini dans la formule de conjugaison précédente :

$$
\overline{\mathrm{H}^{\prime} \mathbf{F}^{\prime}}=\frac{n^{\prime}}{C}=f^{\prime}
$$

Cette quantité est appelée la distance focale image du système, ou parfois tout simplement la focale du système. Elle est positive pour un système convergent.

Le foyer objet a pour image le point à l'infini sur l'axe. Sa position, repérée par rapport au point principal objet, est :

$$
\overline{\mathrm{HF}}=-\frac{n}{C}=f
$$

$f$ est la distance focale objet du système. Elle est égale à l'opposé de la distance focale image lorsque les milieux extrêmes ont même indice $\left(n=n^{\prime}\right)$.

Attention les foyers objet et image ne sont pas conjugués.

\section{* Points nodaux}

Les points nodaux $N$ et $N^{\prime}$ sont des points conjugués pour lesquels le grandissement angulaire vaut 1 . D'après la relation de Lagrange-Helmholtz, le grandissement transversal vaut $n / n^{\prime}$. Ces 
points ne sont différents des points principaux que si les milieux extrêmes n'ont pas le même indice.

Pour déterminer la position du point nodal objet, on reprend les lois de transformation des grandissements angulaires lorsqu'on passe du couple de points conjugués $\left(H, H^{\prime}\right)$ au couple $(N$, $\left.N^{\prime}\right)$ :

$$
\left(g_{\alpha}\right)_{\mathrm{NN}}=1=\left(g_{\alpha}\right)_{\mathrm{HF}}+\frac{C}{n^{\prime}} \overline{\mathrm{HN}}=\frac{n}{n^{\prime}}+\frac{C}{n^{\prime}} \overline{\mathrm{HN}} \text { d'où } \overline{\mathrm{HN}}=\frac{n^{\prime}-n}{C}=f^{\prime}+f
$$

On retrouve bien que $\mathrm{H}$ et $\mathrm{N}$ sont confondus si $n=n^{\circ}$, et on a :

$$
\overline{\mathbf{F N}}=f,
$$

On peut dans le même esprit, à partir de la relation de transformation des grandissements transversaux, déterminer la position du point nodal image et on trouve :

$$
\overline{N^{\prime} F^{\prime}}=-f
$$

\section{* Points antiprincipaux et antinodaux}

Les antiprincipaux $\eta$ et $\eta$ ' sont les points conjugués pour lesquels le grandissement transversal vaut -1 et les antinodaux $v$ et $v^{\prime}$ ceux pour lesquels le grandissement angulaire vaut -1 . On peut montrer que ces points sont symétriques des points principaux et nodaux par rapport au foyer correspondant.

La figure ci-dessous montre un exemple de position relative des points cardinaux pour un système convergent de convergence $\mathrm{C}=0.5 \mathrm{~cm}^{-1}$, dont la distance entre plans principaux vaut $1.5 \mathrm{~cm}$, et pour des milieux extrêmes d'indice $\mathrm{n}=1$ et $\mathrm{n}^{\prime}=1.5$ :

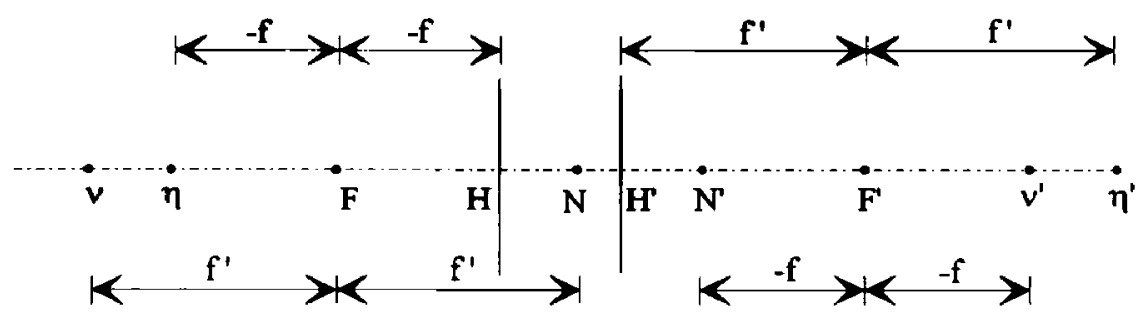

Remarquons que si l'on se donne les positions de $\mathrm{F}, \mathrm{H}, \mathrm{F}$ ' et $\mathrm{H}^{\prime}$, tous les autres points cardinaux s'en déduisent.

3.3.3.2 Construction de l'image d'un objet. Un système optique va être complètement défini en approximation de Gauss par la donnée de ses plans principaux et de ses foyers.

Puisqu'on sait que le système est stigmatique dans l'approximation de Gauss, il suffit pour construire l'image d'un objet B de tracer le cheminement de deux rayons passant par cet objet et de trouver l'intersection $B$ ' des deux rayons émergents. Tous les autres rayons passeront forcément par B'.

On choisit donc deux rayons particuliers passant par B : 
- un rayon incident parallèle à l'axe : le rayon émergent correspondant passe par $F^{\prime}$ et coupe l'incident dans le plan de $\mathrm{H}^{\prime}$;

- un rayon incident passant par $\mathrm{F}$ : le rayon émergent correspondant sort parallèle à l'axe et coupe l'incident dans le plan de $\mathrm{H}$.

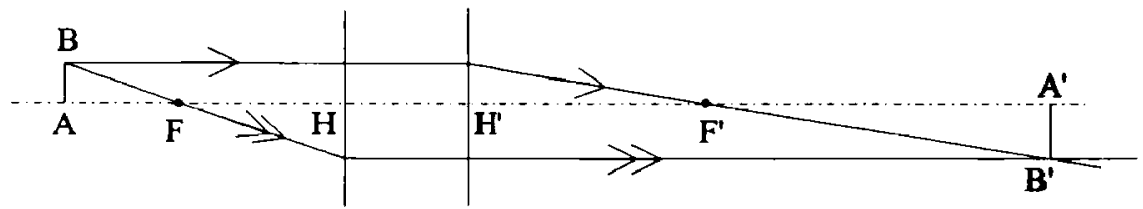

Pour construire l'image d'un point objet sur l'axe A, les deux rayons proposés n'en sont qu'un : c'est l'axe du système. Pour trouver l'image $A^{\prime}$, on utilise le fait que l'image d'une portion de plan orthogonale à l'axe est une portion de plan orthogonale à l'axe. On prend un point $B$ quelconque dans le plan de $A$, on construit son image $B^{\prime}$ et on obtient $A^{\prime}$ sur l'axe dans le plan de $B^{\prime}$.

D'autres rayons particuliers peuvent être utilisés pour construire une image, par exemple un rayon passant par le point nodal objet $\mathrm{N}$ ressortira du système par le conjugué $\mathrm{N}^{\prime}$ et avec un angle égal à l'angle incident :

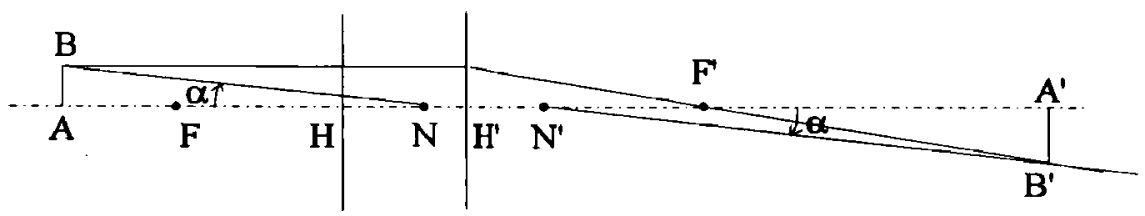

A partir de cette construction, on voit apparaître une expression du grandissement transversal :

$$
\left(g_{y}\right)_{\mathrm{AA}^{\prime}}=\frac{\overline{\overline{\mathrm{A}^{\prime} \mathrm{B}^{\prime}}}}{\overline{\mathrm{AB}}}=\frac{\overline{\overline{\mathrm{N}^{\prime} \mathrm{A}^{\prime}}}}{\overline{\mathrm{NA}}}
$$

On peut également, à partir du tracé d'un rayon issu de $A$, obtenir une expression générale du grandissement angulaire :

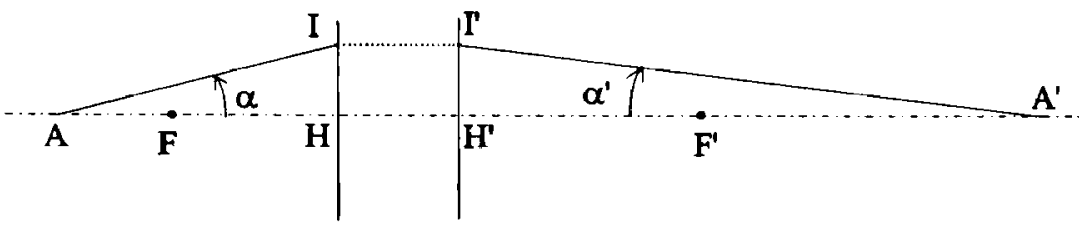


Le rayon incident passe par $A$ et $I$, point du plan principal objet : le rayon émergent passe donc par A' image de A et par I', image de I située dans le plan principal image avec un grandissement transversal de 1 . On obtient alors :

$$
\left(g_{\alpha}\right)_{\mathrm{AA}^{\prime}}=\frac{\alpha^{\prime}}{\alpha}=\frac{\overline{\mathrm{HA}}}{\overline{\mathrm{HA}^{\prime}}}
$$

On peut aussi retrouver sur une construction graphique que les points antiprincipaux $\left(\mathrm{g}_{\mathrm{y}}=\right.$ - 1) sont symétriques des points principaux par rapport aux foyers.

3.3.3.3 Relation de conjugaison de Newton. Il s'agit de relier la position de l'image à celle de l'objet en les repérant par rapport à leur foyer respectif. Ce sont des relations de conjugaison de type différent des formules précédentes puisque les foyers ne sont pas des points conjugués. On peut les obtenir soit avec les formules de conjugaison avec origine aux points principaux en décomposant $\overline{\mathrm{HA}}$ en $\overline{\mathrm{HF}}+\overline{\mathrm{FA}}$ (de même pour $\overline{\mathrm{H}^{\prime} \mathrm{A}^{\prime}}$ ), ou plus simplement en utilisant une construction géornétrique. Reprenons la construction de l'image d'un point $B$ en appelant $I$ et $J$ les points d'intersection des rayons incidents avec les plans principaux :

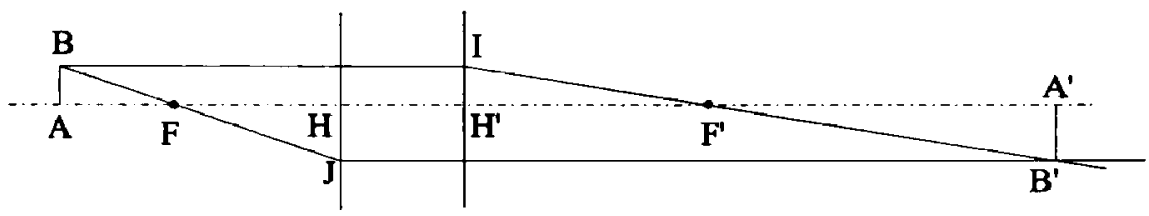

D'après la figure, on a

$$
\begin{aligned}
& \frac{\overline{\mathrm{AB}}}{\overline{\mathrm{FA}}}=\frac{\overline{\mathrm{HJ}}}{\overline{\overline{\mathrm{FH}}}}=-\frac{\overline{\mathrm{A}^{\prime} \mathrm{B}^{\prime}}}{f} \\
& \frac{\overline{\mathrm{A}^{\prime} \mathrm{B}^{\prime}}}{\overline{\mathrm{F}^{\prime} \mathrm{A}^{\prime}}}=\frac{\overline{\mathrm{H}^{\prime} \mathrm{I}}}{\overline{\mathrm{F}^{\prime} \mathrm{H}^{\prime}}}=-\frac{\overline{\mathrm{AB}}}{f^{\prime}}
\end{aligned}
$$

On en déduit la relation de conjugaison de Newton :

$$
\overline{\overline{F A}} \cdot \overline{F^{\prime} A^{\prime}}=f f^{\prime}
$$

ainsi que les expressions du grandissement transversal :

$$
g_{y}=\frac{\overline{\mathrm{A}^{\prime} \mathrm{B}^{\prime}}}{\overline{\mathrm{AB}}}=-\frac{f}{\overline{\mathrm{FA}}}=-\frac{\overline{\mathrm{F}^{\prime} \mathrm{A}^{\prime}}}{f^{\prime}}
$$

On voit là encore que, pour retrouver graphiquement les formules avec origines en des points particuliers, il faut construire des rayons passant par ces points. 
3.3.3.4 Équivalence d'un système focal avec une lentille mince. Si on reprend tout ce qui vient d'être montré sur les systèmes focaux, on voit que tout ce qui concerne l'espace objet est défini par rapport à la position du plan principal objet et du foyer objet, et tout ce qui concerne l'image est défini par rapport au plan principal image et au foyer image. L'écart entre les deux plans principaux intervient comme une translation de l'espace image par rapport à l'espace objet. On peut par exemple s'en convaincre en reprenant les constructions géométriques de rayons ci-dessus.

On va donc pouvoir, dans un premier temps, calculer tout ce qui se passe dans l'espace image en réduisant l'écart entre plans principaux à zéro, ce qui revient par remplacer le système optique par une lentille mince de même focale (éventuellement focale objet et focale image peuvent être différentes si les indices extrêmes sont différents) placée au plan principal objet du système. II suffira ensuite de translater l'espace image de $\mathrm{HH}^{\prime}$ pour trouver toutes les caractéristiques du système en optique de Gauss. Insistons sur le fait que cette méthode n'implique pas d'approximation autre que celle de Gauss.

\subsubsection{Systèmes afocaux}

Il s'agit des systèmes de convergence nulle. Leurs matrices par rapport a deux points conjugués $\mathrm{A}$ et $\mathrm{A}$ ' sont diagonales, de la forme :

$$
\left[\begin{array}{cc}
a_{1} & 0 \\
0 & a_{4}
\end{array}\right] \text { ou encore }\left[\begin{array}{cc}
g_{y} & 0 \\
0 & \frac{n^{\prime}}{n} g_{\alpha}
\end{array}\right]
$$

On les appelle afocaux car ils n'ont pas de foyers (ils sont rejetés à l'infini). En effet si on prend un rayon incident parallèle à l'axe, de coordonnées $(Z, \Omega=0)$, le rayon émergent vaut $\left(Z^{\prime}=a_{1} Z, \Omega^{\prime}=0\right)$, il est donc également parallèle à l'axe.

On a vu également que $\mathrm{C}=0$ implique que les grandissements transversal et angulaire sont indépendants du couple de points choisi, et donc la matrice du système aussi. Comme les grandissements sont reliés par la relation de Lagrange-Helmholtz, le système afocal est finalement caractérisé par la donnée de l'un des grandissements.

\section{Conclusion :}

L'approximation de Gauss est un cadre d'étude de la formation des images au $1^{\text {er }}$ ordre en termes des paramètres des rayons. Elle a l'immense avantage de donner des résultats satisfaisants dans beaucoup de cas tout en étant très simple : les systèmes optiques même complexes se ramènent à quelques paramètres (plans principaux, foyers) ce qui permet alors de raisonner entièrement en lentilles minces, puis on translate les résultats de la distance entre plans principaux. Les caractéristiques des images sont obtenues grâce à des formules de conjugaison linéaires, ou à des constructions graphiques, ou encore à des calculs d'algèbre linéaire utilisant des matrices $2 \times 2$.

Dans le processus de conception d'un système optique, la première étape consistera donc à résoudre le problème en optique de Gauss en utilisant des lentilles minces (même les miroirs peuvent être remplacés par des lentilles à condition de « déplier » les axes). Compte tenu de la fonction de chaque lentille dans le système (par exemple pour quels points conjugués elle travaille, pour quelle ouverture, etc), on pourra la remplacer par un système plus complexe 
dont certaines aberrations seront corrigées. Il faudra finalement prendre en compte la translation $\mathrm{HH}$ ' pour définir les positions relatives des éléments dans le système final.

\section{ANALYSE DES SYSTÈMES OPTIQUES DANS L'APPROXIMATION DE GAUSS}

Les propriétés générales des systèmes centrés ont été vues dans la partie 3. Nous avons vu notamment qu'il suffisait de déterminer foyers et plans principaux pour déterminer complètement un système optique centré : on peut à partir de là retrouver les autres points cardinaux et faire toutes les constructions ; on dispose des relations de conjugaison et du grandissement transversal avec origine aux plans principaux (Descartes) et avec origine aux foyers (Newton), des relations de Lagrange-Helmholtz et de la relation $g_{y}=g_{x} g_{\alpha}$ permettant de trouver les autres grandissements.

Dans cette partie nous allons voir comment retrouver rapidement les foyers et plans principaux des systèmes élémentaires puis de leurs associations. Nous verrons en particulier le cas des systèmes afocaux, et des systèmes catadioptriques (association de systèmes comprenant au moins un miroir).

\subsection{Systèmes élémentaires}

\section{1. I Dioptre plan et miroir plan}

Ce sont des systèmes afocaux élémentaires, de grandissement transversal 1 . On a déjà dit que l'image dans un miroir plan était simplement le symétrique par rapport au plan du miroir. En ce qui concerne le dioptre plan, on retrouve facilement en linéarisant la loi de la réfraction la relation de conjugaison :

$$
x^{\prime} / x=n^{\prime} / n
$$

où $x$ et $x^{\prime}$ sont les distances de l'objet et de l'image au dioptre.

\subsubsection{Dioptre sphérique, lentille mince, miroir sphérique}

Pour tous ces éléments focaux à simple surface, les points de la surface sont leur propre image, les points principaux sont donc confondus avec la surface elle-même (pour les constructions dans l'approximation de Gauss, on remplacera cette surface par son plan tangent au sommet). Si l'on connaît les deux indices extrêmes $n$ et $n$ ', il suffit donc de connaître la convergence.

Pour le dioptre sphérique on a vu au \$3.1.2 que la convergence s'exprimait sous la forme : $C=\left(n^{\prime}-n\right) / R$ avec $R=\overline{\mathrm{SC}}$. Le rayon passant par le centre n'étant pas dévié, on a $\mathrm{C}=\mathrm{N}=\mathrm{N}^{\prime}$. Les foyers, obtenus par exemple à partir de $C=-n^{\prime} / f^{\prime}$ avec $f^{\prime}=\overline{\mathrm{SF}}$, sont toujours situés en-dehors du segment SC.

Pour la lentille mince, elle est en général caractérisée par la valeur de sa focale, on a donc directement les positions de $F$ et $F$ '. Souvent on parle de lentille mince lorsque les milieux extrêmes sont identiques, auquel cas focale objet et focale image sont égales et opposées. Les rayons passant par le centre $O$ de la lentille ne sont pas déviés et on utilise souvent la relation de conjugaison : $1 / x^{\prime}-1 / x=1 / f^{\prime}$ et la relation donnant le grandissement transversal : 
$g_{y}=x / x$, ou $x^{\prime}=\overline{\mathrm{OA}^{\prime}}$ et $x=\overline{\mathrm{OA}}$. Ces relations ne sont autres que les relations avec origine aux plans principaux qui ont été démontrées dans le cas général de l'approximation de Gauss dans la partie 3.

Pour le miroir sphérique, les rayons passant par le centre de courbure ne sont pas déviés. Toutefois à cause du changement de sens de propagation de la lumière, il s'agit cette fois des points antinodaux (ou antiprincipaux puisque les milieux extrêmes ont forcément même indice). On a donc $\mathrm{C}=\eta=\eta$ ' et les foyers $\mathrm{F}$ et $\mathrm{F}^{\prime}$ sont au milieu de $\eta \mathrm{H}$ et $\eta{ }^{\prime} \mathrm{H}^{\prime}$, donc au milieu de SC (à ne pas confondre avec le dioptre sphérique !). La focale est égale en valeur absolue à la moitié du rayon de courbure du miroir, le miroir concave étant convergent, le miroir convexe divergent. Dans les systèmes contenant peu d'éléments, un ou deux miroirs sphériques par exemple, la convention de signe générale adoptée dans les parties 2.4 et 3 n'est pas la plus commode. On aura plutôt envie de choisir un sens positif unique le long de l'axe, indépendamment du sens de propagation de la lumière. Le mieux dans ce cas est d'utiliser les constructions pour déterminer les signes, en prenant garde au fait que des signes seront modifés par rapport aux relations générales vues dans la partie 3.

\subsection{Association de deux systèmes centrés dioptriques}

\subsubsection{Méthode graphique}

La méthode graphique générale consiste à placer sur un schéma, de préférence à l'échelle, les plans principaux et foyers (éventuellement sommets et centres) des sous-systèmes. On construit à partir de là le trajet d'un rayon incident parallèle à l'axe, d'où l'on obtient le foyer image du système (intersection du rayon émergent avec l'axe) et le plan principal. image (intersection du rayon incident et de l'émergent correspondant). Puis on construit le trajet inverse d'un rayon qui émerge du système parallèlement à l'axe, d'où l'on obtient $\mathrm{F}$ et $\mathrm{H}$. On peut alors vérifier que la relation entre $\overline{\mathrm{HF}}$ et $\overline{\mathrm{H}^{\prime} \mathrm{F}^{\prime}}$ est bien satisfaite, ce qui donne une confirmation de la construction. Les imprécisions de la construction font qu'il est ensuite préférable de confirmer les positions des points cardinaux par une méthode de calcul de ces positions.

Pour certains systèmes particuliers, d'autres tracés de rayons peuvent donner plus rapidement les points cardinaux. C'est le cas des systèmes centrés symétriques qui possèdent, en plus de la symétrie de révolution autour de l'axe optique, un plan de symétrie orthogonal à l'axe optique, y compris du point de vue des indices de réfraction. Une lentille épaisse biconvexe ou biconcave dont les faces ont même rayon de courbure, une lentille boule, l'association de deux lentilles minces de même focale, en sont quelques exemples (dans le cas où les milieux extrêmes ont même indice).

Si l'on appelle $O$ l'intersection du plan de symétrie et de l'axe optique, ce point est le centre optique du système. Ce centre optique a pour propriété que tout rayon passant par $\mathrm{O}$ correspond dans les espaces objet et image du système global à des rayons de même direction.

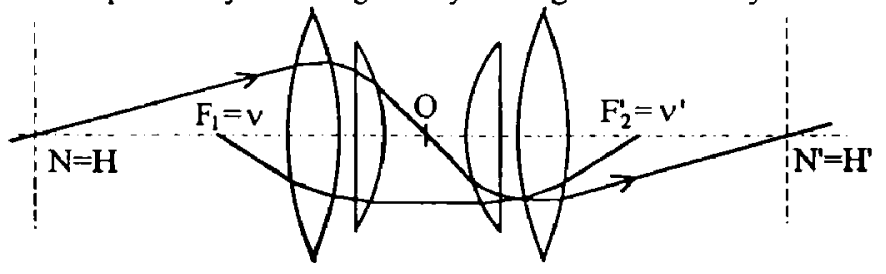


Il suffit alors de calculer l'image de $\mathrm{O}$ à travers la deuxième moitié du système pour trouver le point nodal image (confondu avec le principal image du fait de la symétrie des indices). Le point nodal objet (confondu avec le principal objet) est symétrique du point nodal image par rapport à $\mathrm{O}$.

Les antiprincipaux s'obtiennent également facilement pour un système symétrique. En effet considérons un rayon parallèle à l'axe dans l'espace contenant le plan de symétrie du système : il correspond à des rayons incident et émergent d'angles opposés. D'autre part, il provient du foyer objet de la première moitié du système et émerge en passant par le foyer image de la deuxième moitié du système. $F_{1}$ et $F_{2}^{\prime}$ sont donc les antinodaux (et antiprincipaux) du système global.

\subsubsection{Calcul des positions des points cardinaux}

4.2.2. 1 A partir des constructions. On peut calculer les positions de F, F', $H$ et $H^{\prime}$ à partir des constructions de rayons précédentes, en écrivant des relations entre triangles semblables. On déterminera alors les points cardinaux du système global à partir des points cardinaux des soussystèmes que l'on aura utilisé pour la construction graphique. Ceci est d'ailleurs la base des autres méthodes qui suivent, puisqu'on sait retrouver par des constructions la plupart des formules de conjugaison.

4.2.2.2 A partir des formules de conjugaison. On utilise les formules de conjugaison (Newton ou Descartes) pour calculer les foyers objet et image : on calcule l'image d'un point objet à l'infini sur l'axe à travers les deux sous-systèmes, ce qui revient à calculer l'image de $F_{1}$ à travers le deuxième système ; on cherche l'objet dont l'image à travers le système est à l'infini, ce qui revient à déterminer l'objet dont l'image à travers le premier sous-système est $F_{2}$, foyer objet du deuxième système. Pour $\mathrm{H}_{\text {et }} \mathrm{H}^{\prime}$, on pourra utiliser la focale du système global, calculée à partir de deux relations que nous allons voir maintenant.

4.2.2.3 Calcul de la focale. On peut par exemple faire apparaître la focale objet du système en considérant un objet à l'infini de diamètre apparent $\theta$ et en calculant la taille $y^{\prime}$ de son image à travers le système global $\left(y^{\prime}=-f^{\theta}\right)$. On calcule par ailleurs $y^{\prime}$ à partir de la focale objet du premier système et du grandissement transversal du deuxième et on obtient :

$$
f=f_{1} \cdot\left(g_{y}\right)_{2 \text { pour } F_{1} \mathrm{~F}}
$$

Cette relation est souvent utilisée aussi pour l'étude de télescopes, où l'association de deux miroirs sphériques donne un système équivalent à un système dioptrique. On peut la généraliser au cas d'un système constitué de plusieurs systèmes centrés $S_{1}$ à $S_{n}$, si l'on connaît la position des images intermédiaires $A_{2}$ à $A_{n}$ correspondant à un objet $A_{1}$ à l'infini sur l'axe. Pour le système $S_{i}$, appelons $x_{i}$ la position de l'objet $A_{i}$ par rapport à son plan principal objet, $x_{i}^{\prime}$ la position de l'image $A_{i}^{\prime}$ par rapport à son plan principal image et $\left(g_{y}\right)_{i}$ le grandissement transversal correspondant. La focale objet du système global peut s'écrire :

$$
f=f_{1}\left(g_{y}\right)_{2} \cdots\left(g_{y}\right)_{n}
$$


Dans la relation précédente, on peut exprimer $\left(g_{y}\right)_{2 \text { pour } F_{1} F}$ à partir des formules de Newton, en faisant apparaître la focale objet du deuxième système et la distance $\overline{F_{2} F_{1}^{\prime}}$, qui caractérise l'écart entre les deux systèmes. On peut aussi exprimer cette distance en fonction des focales et de l'écart $e=\overline{\mathrm{H}_{1}, \mathrm{H}_{2}}$ entre le plan principal image du premier sous-système et le plan principal objet du deuxième. On obtient après quelques manipulations la formule de Gullstrand, qui donne la convergence d'un système en fonction de celles des sous-systèmes le composant :

$$
C=C_{1}+C_{2}-\frac{e}{n_{i}} C_{1} C_{2}
$$

où $n_{\mathrm{i}}$ est l'indice du milieu intermédiaire séparant les deux sous-systèmes. Cette relation peut s'appliquer au cas de systèmes composés de miroirs à condition de prendre des précautions de signe (comme toujours avec les miroirs!). Une possibilité pour éviter les erreurs de signe consiste à déplier le système en remplaçant un miroir de rayon de courbure $\mathrm{R}$ par une lentille mince de focale $R / 2$ (convergente si le miroir est concave, divergente s'il est convexe). Il est cependant indispensable de faire une figure pour vérifier la cohérence du résultat obtenu.

\subsubsection{Méthode matricielle}

Cette méthode peut être utile dans le cas de systèmes complexes qui ne présentent pas de propriétés de symétrie particulières ou pour lesquels on souhaite varier les paramètres (rayon des dioptres, espacements entre les dioptres, indices, etc.) de façon systématique. Il s'agit typiquement de la méthode à employer dans un programme de calcul optique où l'utilisateur rentre les paramètres de son système optique et veut obtenir les points cardinaux, faire un tracé de rayons, déterminer la position et la taille de l'image, etc.

Cette méthode est basée sur l'utilisation des matrices qui ont été vues dans la partie 3 sur l'approximation de Gauss. Il s'agit donc ici de déterminer les caractéristiques d'un système centré dans le domaine paraxial. Nous ne redonnerons pas ici les justifications de la méthode (se reporter à la partie 3).

Le principe de base est le suivant

- on calcule la matrice $M_{E S}$ du système entre sa face d'entrée et sa face de sortie, en multipliant (dans le bon ordre !) les matrices des différents éléments simples qui le constituent

$$
\mathbf{M}_{\mathrm{ES}}=\left[\begin{array}{ll}
a_{1} & a_{2} \\
a_{3} & a_{4}
\end{array}\right]=\mathbf{M}_{n} \cdot \mathbf{M}_{n-1} \cdots \mathbf{M}_{1}
$$

Rappels des différentes matrices :

\begin{tabular}{|l|c|}
\hline $\begin{array}{l}\text { espace libre de longueur d dans un milieu } \\
\text { d'indice } n\end{array}$ & {$\left[\begin{array}{cc}1 & \mathrm{~d} / \mathrm{n} \\
0 & 1\end{array}\right]$} \\
\hline dioptre sphérique $n \boldsymbol{n}^{\prime}$ de rayon $R$ & {$\left[\begin{array}{cc}1 & 0 \\
-\left(\mathrm{n}^{\prime}-\mathrm{n}\right) / \mathrm{R} & 1\end{array}\right]$} \\
\hline lentille dans l'air de focale image f & {$\left[\begin{array}{cc}1 & 0 \\
-1 / \mathrm{f}^{\prime} & 1\end{array}\right]$} \\
\hline
\end{tabular}


miroir de rayon de courbure $R$ donc de convergence $|C|=2 n /|R|$, avec $\mathrm{C}>0$ pour un miroir concave et $\mathrm{C}<0$ pour un miroir convexe

$$
\left[\begin{array}{cc}
1 & 0 \\
-\mathrm{C} & 1
\end{array}\right]
$$

- on obtient immédiatement la convergence (et donc les focales puisqu'on connaît les indices) qui est l'opposé du terme $a_{3}$ de la matrice

- on obtient les positions des points principaux par rapport aux faces d'entrée et de sortie du système en écrivant la relation entre la matrice $\mathbf{M}_{\mathrm{ES}}$ et la matrice $\mathbf{M}_{\mathrm{HH}}$ qui a une forme très simple :

$$
\begin{gathered}
\mathbf{M}_{\mathrm{HH}}=\left[\begin{array}{cc}
1 & 0 \\
-C & 1
\end{array}\right] \\
\mathbf{M}_{\mathrm{ES}}=\left[\begin{array}{cc}
1 & \overline{\mathrm{HS}} / n^{\prime} \\
0 & 1
\end{array}\right] \mathbf{M}_{\mathrm{HH}} \cdot\left[\begin{array}{cc}
1 & \overline{\mathrm{EH}} / \boldsymbol{n} \\
0 & 1
\end{array}\right]=\left[\begin{array}{cc}
1-C \overline{\mathrm{HS}^{\prime}} / n^{\prime} & * \\
-C & 1-C \overline{\mathrm{EH}} / n
\end{array}\right]
\end{gathered}
$$

En identifiant les termes $a_{1}$ et $a_{4}$, on obtient $\overline{\mathrm{H}^{\prime} \mathrm{S}}$ et $\overline{\mathrm{EH}}\left(n, n^{*}:\right.$ indices des milieux extrêmes)

- on peut réaliser un tracé de rayons à partir de la matrice $\mathbf{M}_{\mathrm{ES}}$ : il suffit de multiplier les coordonnées du rayon incident dans le repère d'origine $\mathrm{E}$ par la matrice pour obtenir le rayon émergent dans le repère d'origine $\mathrm{S}$. $\mathrm{Si}$ on veut les rayons intermédiaires, il suffit de décomposer dans les matrices intermédiaires.

- on peut calculer la position et la taille de l'image $A^{\prime} B^{\prime} d^{\prime}$ 'un objet $\mathrm{AB}$ à travers le système en écrivant la matrice $\mathbf{M}_{\mathrm{AA}}$ ' à partir de $\mathbf{M}_{\mathrm{ES}}$ : on annule le coefficient $a_{2}$ de $\mathbf{M}_{\mathrm{AA}}$ (ce qui donne la relation de conjugaison) et son coefficient $a_{1}$ donne le grandissement transversal.

\subsection{Systèmes afocaux}

Ils sont définis par le fait que leur convergence est nulle, ou en d'autres termes par le fait qu'ils donnent d'un objet à l'infini une image à l'infini. Les systèmes afocaux simples que sont le dioptre plan, la lame à faces planes et parallèles et le miroir plan peuvent être traités de façon élémentaire.

Pour tous les systèmes focaux non élémentaires, on pourra les étudier en les décomposant en deux sous-systèmes focaux, avec le foyer image du premier système confondu avec le foyer objet du second.

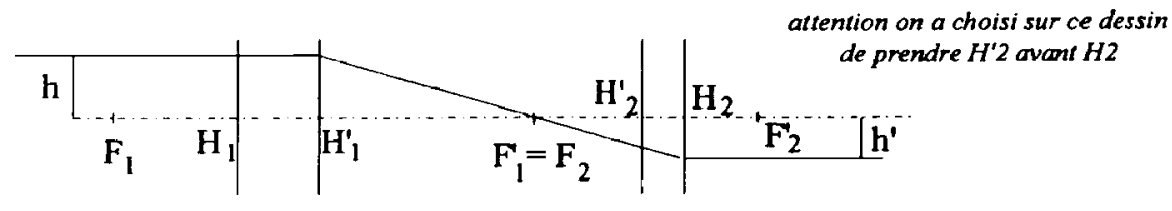

On peut dans ce cas retrouver la valeur du grandissement transversal d'après la figure 


$$
g_{y}=\frac{h^{+}}{h}=\frac{\overline{\mathrm{H}_{2} \mathrm{~F}_{2}}}{\overline{\mathrm{H}_{1}^{\prime} \mathrm{F}_{1}^{\prime}}}=\frac{f_{2}}{f^{\prime}}
$$

On peut également obtenir la relation de conjugaison, par exemple en utilisant la relation démontrée au $\$ 3.3 .2$ :

$$
\frac{x^{\prime}}{x}=\frac{n^{\prime}}{n} g_{y}{ }^{2}=\frac{f_{2} f_{2}^{\prime}}{f_{1} f_{1}^{\prime}}
$$

où $\boldsymbol{x}$ et $\boldsymbol{x}^{\prime}$ sont les coordonnées des points objet et image par rapport à $\mathbf{n}^{\prime}$ importe quel couple d'autres points conjugués (par exemple $F_{1}$ et $F_{2}^{\prime}$ ).

Notons que cette décomposition en deux sous-systèmes n'est pas unique, on pourra donc choisir la mieux adaptée.

\subsection{Systèmes catadioptriques}

Il s'agit de systèmes constitués de dioptres et d'au moins un miroir.

\subsubsection{Système optique équivalent}

4.4.1.1 Système catadioptrique à un seul miroir. Considérons le cas général d'un système limité à droite par un miroir sphérique :

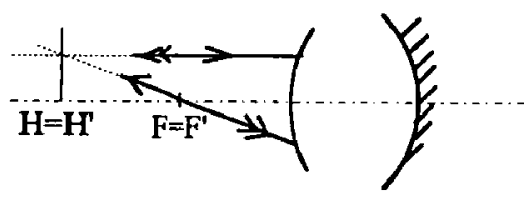

Un rayon incident parallèle à l'axe va traverser la partie dioptrique du système, se réfléchir sur le miroir et retraverser la partie dioptrique. Le rayon émergent correspondant va couper l'axe en un point qui est le foyer image $F$ ' du système catadioptrique.

Suivons maintenant ce même rayon en sens inverse. Par propriété de retour inverse de la lumière, il va ressortir parallèle à l'axe. Le point F' est donc également le foyer objet du système catadioptrique

On peut faire le même raisonnement pour les plans principaux (cf figure) : les plans principaux objet et image sont également confondus.

Finalement un système catadioptrique à un miroir est équivalent à un miroir sphérique de sommet $\mathbf{S}=\mathbf{H}=\mathrm{H}^{\prime}$ et de foyers $\mathbf{F}=\mathrm{F}^{\prime}$.

4.4.1.2 Système catadioptrique à deux miroirs. Il s'agit par exemple d'un télescope, le deuxième miroir devant être percé au centre pour que la lumière puisse sortir du système. Prenons par exemple le cas d'un télescope de Cassegrain : 


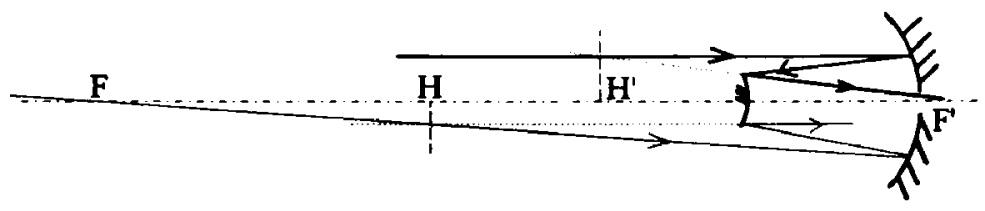

La lumière émergente se propage dans le même sens que l'incidente. On peut déterminer les plans principaux et les foyers du système, qui est équivalent à un système dioptrique. En particulier l'image à travers un tel système est superposable à l'objet.

De façon générale, un système comprenant un nombre impair de miroirs sera équivalent à un miroir, tandis qu'un système comprenant un nombre pair de miroirs sera équivalent à un système dioptrique

\subsubsection{Détermination du miroir ṕquivalent à un système catadioptrique à un seul miroir}

4.4.2. 1 Recherche du sommet du miroir équivalent. Nous avons vu qu'un moyen de trouver le sommet du miroir équivalent consiste à déterminer le plan principal image du système catadioptrique en traçant le cheminement d'un rayon incident parallèle à l'axe. Nous allons voir ici une autre méthode généralement plus rapide.

Considérons un rayon incident tel qu'après traversée de la partie dioptrique du système, il passe par le sommet $\mathrm{S}$ du miroir. Il va être réfléchi sur le miroir symétriquement par rapport à l'axe optique du système.

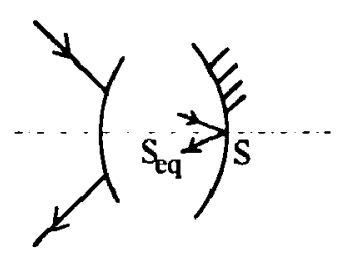

Compte tenu de la symétrie de révolution du système, le rayon émergent va forcément être symétrique de l'incident par rapport à l'axe. Ces deux rayons se coupent en un point $S$ ' de l'axe. Ce point $S_{\text {eq, }}$, confondu avec son image à travers le système catadioptrique, est tel qu'un rayon incident passant par $S_{\llcorner q}$ ressort du système symétriquement par rapport à l'axe optique c'est le sommet du miroir équivalent. D'autre part, d'après la façon dont on a construit $S_{\text {eq, }}$ c'est le point dont l'image à travers la partie dioptrique du système est $\mathrm{S}$ pour la lumière se propageant de gauche à droite. Ou inversement

Le sommet $S_{\mathrm{eq}}$ du miroir équivalent est l'image du sommet $S$ du miroir réel à travers la partie dioptrique du système, pour la lumière se propageant de droite à gauche (sens de la lumière réfléchie).

4.4.2.2 Recherche du centre du miroir équivalent. On a vu une méthode graphique permettant de trouver le foyer du miroir équivalent. On pourrait l'appliquer pour trouver le centre puisqu'on sait déterminer le sommet. Nous allons voir ici une autre méthode plus rapide. 
Considérons un rayon incident tel qu'après traversée de la partie dioptrique du système, il passe par le centre $C$ du miroir. Il va être réfléchi sur lui-même par le miroir, donc le rayon est superposé à l'incident.

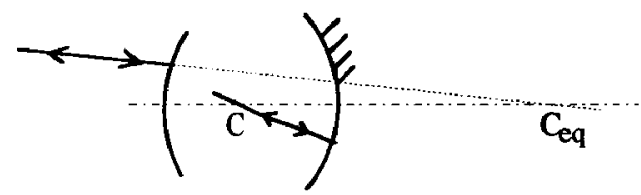

Le point $C_{e q}$ où ce rayon incident coupe l'axe du système est donc tel qu'un rayon incident passant par $\mathrm{C}_{\mathrm{eq}}$ revient sur lui-même : c'est le centre du miroir équivalent. On a donc :

Le centre $C_{\text {eq }}$ du miroir équivalent est l'image du centre $C$ du miroir réel à travers la partie dioptrique du système, pour la lumière se propageant de droite à gauche (sens de la lumière réfléchie).

\subsubsection{Systèmes catadioptriques afocaux}

Nous allons regarder les cas particuliers où le sommet ou le centre du miroir équivalent est à l'infini.

4.4.3.1 Systèmes équivalents à un miroir plan. Dans le cas où le centre du miroir équivalent est à l'infini, le système est équivalent à un miroir plan. C'est un système afocal. Ceci se produit lorsque le centre $\mathrm{C}$ du miroir réel est confondu avec le foyer image de la partie dioptrique du système :

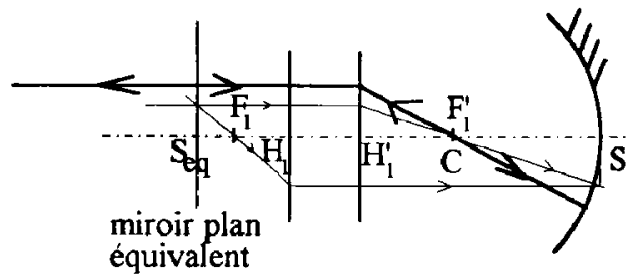

Pour ce système afocal, le grandissement transversal vaut +1 pour tous les points.

4.4.3.2 Systèmes équivalents à un miroir sphérique placé à l'infini. Considérons le cas où le foyer de la partie dioptrique du système est confondu avec le sommet $S$ du miroir réel. Le sommet S' du miroir équivalent est situé à l'infini, tandis que son centre C' est à distance finie. 


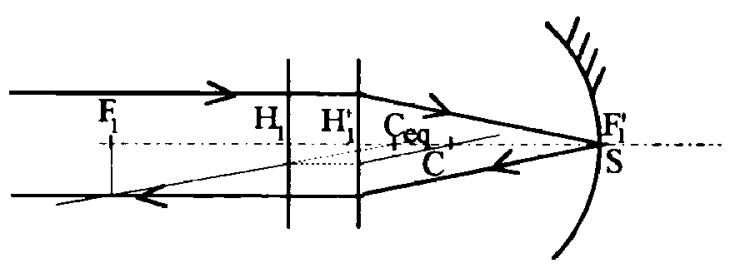

Pour ce système afocal, le grandissement transversal vaut $\mathbf{- 1}$.

Ce type de système n'est pas réalisable à l'aide d'un miroir unique. Il est encore appelé ouil de chat. Il a des propriétés intéressantes :

(i) l'image par le système d'un point $B$ quelconque est le point B', symétrique de $B$ par rapport au centre $\mathrm{C}_{\mathrm{eq}}$ du miroir équivalent ;

(ii) tout rayon incident ressort parallèle à lui-même.

On peut montrer la propriété (i) à partir de la relation de conjugaison du miroir sphérique avec origine au centre et SC infini :

$$
\frac{1}{\overline{C_{e q} A}}+\frac{1}{\overline{C_{e q} A^{\prime}}}=0 \quad \text { soit: } \overline{C_{e q} A^{\prime}}=-\overline{C_{e q} A}
$$

Pour montrer la propriété (ii), on peut toujours considérer qu'un rayon incident faisant un angle $\alpha$ avec l'axe optique passe par un point à l'infini $S_{\alpha}$ dans la direction $\alpha$, où dans notre cas $S_{\alpha}$ est dans le plan du miroir. Or un point de la surface du miroir a pour image lui-même. Le rayon émergent doit donc lui aussi passer par $S_{\alpha}$, c'est-à-dire avoir la même direction que le rayon incident.

Citons comme exemple de système catadioptrique de ce type, une lentille plan convexe telle que le foyer du dioptre se trouve sur la face plane métallisée

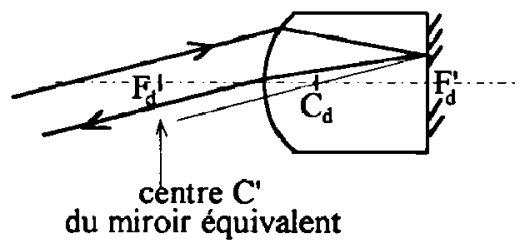

La propriété de retour de la lumière dans la direction incidente quelle qu'elle soit rend un tel système très pratique pour la réalisation de cataphotes (visualisation automobile par ex).

Une autre configuration souvent utilisée est constituée d'une lentille convergente et d'un miroir plan au foyer image de la lentille : 


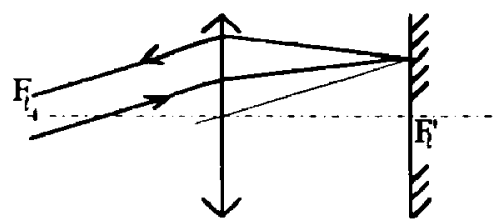

\section{PROPRIÉTÉS DES INSTRUMENTS : GRANDISSEMENT, PUISSANCE, GROSSISSEMENT}

Nous allons maintenant utiliser les bases d'optique géométrique que nous avons détaillées jusqu'ici pour analyser et concevoir des instruments d'optique, c'est-à-dire des systèmes optiques adaptés à une fonction donnée : observation de petits objets (loupe, oculaire, microscope), photographie, observation d'objets très éloignés (jumelles, lunette astronomique, télescope), etc.

Dans cette première partie d'optique instrumentale, nous allons définir un certain nombre de propriétés qui vont permettre de qualifier et de comparer les instruments, puis nous détaillerons les propriétés relatives à la grandeur de l'image formée par l'instrument. Certaines de ces définitions restent valables hors de l'approximation de Gauss, toutefois en ce qui concerne les calculs, nous nous limiterons en général à cette approximation.

\subsection{Classification et qualification des instruments}

On va s'intéresser ici aux instruments destinés à former des images. Il existe également toute une catégorie d'instruments qui servent à transporter ou concentrer l'énergie électromagnétique transportée par le rayonnement (par exemple les systèmes d'éclairage) que nous n'évoquerons pas ici.

Parmi les instruments destinés à former des images, on distingue en général deux catégories :

\subsubsection{Les instruments de projection}

Ils sont parfois appelés instruments objectifs. Ils forment d'un objet une image réelle sur un écran, ou sur un détecteur photoélectrique (photodiode, photomultiplicateur, matrice de CCD), ou sur une pellicule photographique. C'est le cas par exemple des appareils photos, des projecteurs de cinéma ou des télescopes (sans oculaire) associés à un détecteur CCD.

\subsubsection{Les instruments oculaires (ou visuels, ou subjectifs)}

Ils sont destinés à être associés à l'œil pour faciliter l'observation (en général augmenter la taille de l'image). Ils doivent donner une image qui est en général à l'infini ou virtuelle que l'œil transforme en une image réelle sur sa rétine. Leurs propriétés vont donc être liées et 
comparées à celles de l'œil, c'est pourquoi nous rappellerons un peu plus loin les propriétés principales de l'œil, qui est lui-même un instrument très sophistiqué.

Parmi ces instruments oculaires, on distingue encore deux types de fonctions, suivant qu'ils sont destinés à l'observation de petits objets rapprochés (type microscope) ou à l'observation d'objets très éloignés (type lunette astronomique ou télescope avec oculaire).

\subsubsection{Qualités d'un instrument}

Nous qualifierons un instrument par les propriétés suivantes :

(i) la grandeur de l'image par rapport à celle de l'objet, qui sera définie de façon différente suivant la nature de l'objet observé (proche ou éloigné) et de l'instrument (projection ou oculaire) ;

(ii) le champ de l'instrument, à savoir la portion d'espace qui est vue à travers l'instrument: nous distinguerons le champ en largeur (surface dans le plan de l'objet) et la profondeur de champ (distance le long de l'axe du système optique, autour de l'objet);

(iii) le pouvoir séparateur, qui est la capacité de l'instrument à séparer les détails de l'objet ;

(iv) la clarté et, de façon plus générale, toutes les propriétés photométriques de l'instrument.

La première propriété sera étudiée en détail dans la suite de cette partie. Les champs feront l'objet de la partie suivante. Le pouvoir séparateur est une notion plus complexe, qui fait intervenir les aberrations du système optique, les phénomènes de diffraction, ainsi que les propriétés du détecteur auquel est associé l'instrument (pouvoir séparateur de l'œil, grain de la pellicule photo, taille d'un élément $\mathrm{CCD}$ ). Du point de vue géométrique, c'est l'ouverture du système optique, dont nous parlerons lors de l'étude des champs, qui peut influencer le pouvoir séparateur d'un instrument. Enfin nous verrons dans la dernière partie de ce cours les propriétés photométriques des instruments, qui sont elles aussi liées à l'étude des champs.

\subsection{Rappels sur les propriétés de l'œil}

Cette partie a pour objet de rappeler certaines propriétés de l'œil qui seront utiles pour la qualification des instruments oculaires. De plus l'œil est lui aussi un instrument d'optique complexe, et certaines notions évoquées pour l'œil (résolution, pupille) se retrouveront par la suite dans les propriétés des instruments.

\subsection{Le système optique de l'oul}

Les propriétés de convergence de l'œil sont dues à la cornée (dioptre air/eau de rayon de courbure $8 \mathrm{~mm}$ ) et au cristallin (lentille biconvexe convergente baignée dans du liquide, $d$ 'indice légèrement supérieur à celui de l'eau, et de focale variable ce qui permet l'accommodation). Ce système optique est associé à un détecteur qui est la rétine, dont la seule partie qui nous intéresse pour la formation des images est la fovéa (partie légèrement décalée par rapport à l'axe du système optique).

5.2.1.1 Dioptre sphérique équivalent. Lorsqu'on calcule les points cardinaux du système optique de l'œil, on trouve que les points nodaux $N$ et $N^{\prime}$ sont quasiment confondus. $P a r$ conséquent les points principaux $\mathrm{H}$ et $\mathrm{H}^{\prime}$ sont également confondus, mais distincts de $\mathrm{N}=\mathrm{N}^{\prime}$ puisque les indices des milieux extrêmes sont différents. Le système est donc équivalent à un 
dioptre sphérique air/eau de centre $\mathrm{C}=\mathrm{N}=\mathrm{N}^{\prime}$ et de sommet $\mathrm{S}=\mathrm{H}=\mathrm{H}^{\prime}$. La disposition est la suivante :

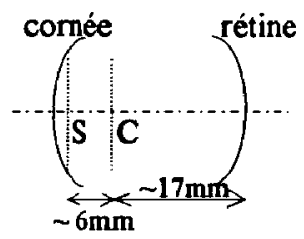

Pour l'œil normal au repos, le foyer image du système se trouve sur la rétine (vision nette à l'infini), la convergence vaut 60 dioptries, ce qui correspond à un rayon de courbure du dioptre équivalent de $5.6 \mathrm{~mm}$. Lorsque l'ceil accommode, sa convergence augmente, le foyer image du système se rapproche de $\mathrm{C}$, le rayon de courbure du dioptre équivalent diminue $(5.2 \mathrm{~mm}$ environ pour une accommodation à $25 \mathrm{~cm}$ ). Cette variation se fait par une variation de la convergence du cristallin, les points $S$ et $C$ se déplacent de quelques dixièmes de $\mathrm{mm}$, ce qu'on peut quasiment considérer comme fixe par rapport à la distance la plus proche à l'objet observé.

5.2.1.2 Accommodation. L'ceil «normal» ou emmétrope voit sans fatigue à l'infini (ponctum remotum $\mathrm{D}=\infty$ ) et peut accommoder jusqu'à une distance typique $\mathrm{d}=25 \mathrm{~cm}$ (ponctum proximum). Lorsque cette plage d'accommodation est différente, on dit que l'œil est amétrope. Diffërentes amétropies peuvent se présenter parmi lesquelles

- la myopie : l'cil est trop convergent, il voit net de $\mathrm{d}<25 \mathrm{~cm}$ à une distance $\mathrm{D}$ finie ;

- l'hypermétropie : l'œil n'est pas assez convergent, il voit net de $\mathrm{d}>25 \mathrm{~cm}$ à $\mathrm{D}<0$ (ceci signifie qu'il peut voir un objet virtuel par rapport à son œil)

- la presbytie : l'œil perd ses facultés d'accommodation, il voit net de $\mathrm{d}>25 \mathrm{~cm}$ à l'infini ; son amplitude dioptrique d'accommodation $A=1 / \mathrm{d}-1 / \mathrm{D}$ tend vers zéro, alors qu'elle vaut 4 dioptries (ou $\mathrm{m}^{-1}$ ) pour un cil normal

5.2.1.3 Taille de l'image. On a vu que le centre et le sommet du dioptre équivalent se déplacent très peu lors de l'accommodation. Ceci implique que la taille $y^{\prime}$ de l'image sur la rétine ne dépend que de l'angle $\theta$ sous lequel est vu l'objet depuis l'œil. En effet, si l'on considère par exemple que le centre est fixe à une distance $\mathrm{a}=17 \mathrm{~mm}$ de la rétine, la taille de l'image formée sur la rétine vaut $y^{\prime}=a \theta$ et ne dépend que de $\theta$ sous lequel est vu l'objet observé depuis le point $\mathrm{C}$.

Si l'objet observé est à l'infini, cet angle est simplement le diamètre apparent de l'objet, indépendant de la position de l'œil. Pour un objet à distance finie de taille $y$, on aura intérêt à placer l'objet au ponctum proximum pour que l'angle sous lequel il est vu par l'œil soit le plus grand possible $\left(\theta_{\max }=\mathrm{y} / 25 \mathrm{~cm}\right.$ pour l'œil normal) : l'image sur la rétine sera alors la plus grande possible. Un instrument sera intéressant s'il augmente l'angle $\theta$ au-delà de ce $\theta_{\max }$.

5.2.1.4 Limite de résolution. Il n'existe pas une réponse unique à la valeur de la limite de résolution de l'œil, car elle dépend de nombreux facteurs : forme des détails observés, couleur, 
luminance, contraste, rayon de la pupille de l'œil, fatigue, etc. On peut cependant en donner un ordre de grandeur : $\theta_{\min }=1$ à $2^{\prime}$. Ceci signifie que pour un objet à distance finie la taille minimale discernable sera $\theta_{\min } \times 25 \mathrm{~cm} \approx 100 \mu \mathrm{m}$, d'où la nécessité d'instruments du type loupe ou microscope pour voir des objets plus petits.

\subsubsection{Pupille de l'ail}

L'ceil comprend un iris de diamètre variable situé entre la cornée et le cristallin. C'est lui qui limite l'ouverture des rayons dans le système : c'est ce qu'on appellera dans l'étude générale des champs le diaphragme d'ouverture du système optique. Son image à travers la cornée (c'est ce qu'on voit quand on regarde l'œil de quelqu'un d'autre) est la pupille d'entrée de l'œil. Le diamètre de cette pupille d'entrée varie entre l et $4 \mathrm{~mm}$ (typiquement $2 \mathrm{~mm}$ en vision diurne).

Ce paramètre est important pour la conception d'un instrument oculaire : on aura souvent intérêt à ce que l'ceil ne limite pas l'ouverture des rayons à travers l'ensemble instrument+ceil, ce qui revient à dire que la pupille de sortie de l'instrument (image de sa pupille d'entrée) devra être plus petite que celle de l'œil (sauf pour des questions de clarté que nous discuterons dans la partie 7.3).

\subsubsection{Champ de l'oil}

Il est déterminé par la dimension de la fovéa, partie de la rétine où on peut obtenir des images nettes. Lorsque l'œil est fixe ce champ est très faible (ouverture totale $2 \omega=1^{\circ}$ ), mais grâce à la mobilité de l'œil dans son orbite ce champ vaut au total $2 \omega=40$ à $50^{\circ}$. Il n'y aura pas d'intérêt à augmenter le champ image d'un instrument oculaire au-delà de cette valeur.

\subsection{Grandeur de l'image : grandissement, puissance, grossissement.}

Il s'agit de qualifier le lien entre la dimension de l'objet et la dimension de l'image formée par l'instrument. Suivant la nature de l'objet (proche ou à l'infini) et suivant la nature de l'instrument (projection ou oculaire), ces dimensions pourront être données par une longueur ou par un angle, d'où la définition de quantités ayant des définitions et même des unités différentes.

\subsubsection{Grandissement (transversal)}

C'est le paramètre qui caractérise les instruments de projection pour lesquels l'image est réelle, donc caractérisée par sa taille $y^{\text {". }}$

Dans le cas d'un objet à distance finie défini par sa taille $y$, il s'agit simplement du grandissement transversal $\mathrm{gy}_{\mathrm{y}}$ du système optique pour les deux points conjugués à utiliser.

Dans le cas d'un objet à l'infini défini par son diamètre apparent $\theta$, le grandissement est remplacé par la quantité $y^{\prime} / \theta=-f$ focale objet du système. 


\subsubsection{Puissance}

C'est un paramètre qui caractérise un instrument oculaire destiné à observer des objets rapprochés. La puissance est définie comme le rapport :

$$
P=\frac{\theta^{\prime}}{y}
$$

entre l'angle sous lequel est vue (par l'œil) l'image formée par l'instrument et la taille $y$ de l'objet. Ce paramètre peut dépendre a priori du réglage de l'instrument (position de l'image) et de la position de l'oeil.

5.3.2.1 Cas d'une image à l'infini. C'est le réglage de l'instrument pour l'œeil normal n'accommodant pas. Dans ce cas, les deux quantités $\theta^{\prime}$ et $y$ sont indépendantes de la position de l'œil.

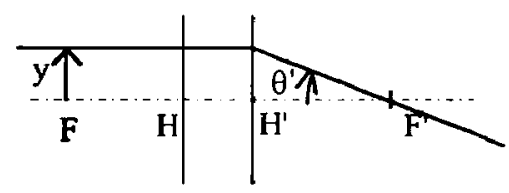

La puissance vaut alors : $P=-\frac{1}{f^{\prime}} \quad$ où $f^{\prime}$ est la focale image du système.

Pour ce réglage de l'instrument, la puissance ne dépend que des propriétés de l'instrument : c'est sa puissance in trinsèque $P_{i}$.

5.3.2.2 Cas d'une image à distance finie. Puisqu'il s'agit toujours d'un instrument oculaire, cette image formée par l'instrument doit être placée entre le ponctum proximum et le ponctum remotum de l'ail. Elle sera à distance finie par exemple dans le cas où l'utilisateur est amétrope ou si l'observateur ernmétrope a accommodé lors du réglage de l'instrument. Dans ce cas, l'angle $\theta$ ' va a priori dépendre du réglage et de la position de l'œil. On peut déterminer l'expression de la puissance dans ce cas à partir du schéma suivant :

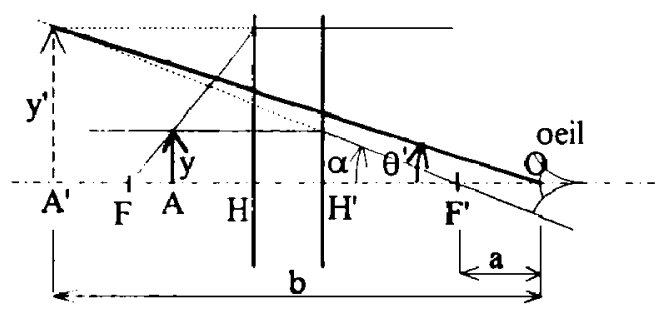


La position de l'œil par rapport au système est caractérisée par la distance au foyer image de l'instrument $a=\overline{F^{\prime} \mathrm{O}}$, et la position de l'image est caractérisée par sa distance à l'œil $b=\overline{\mathbf{A}^{\prime} \mathrm{O}}$.

En utilisant la figure, on a :

$$
\alpha=-\frac{y}{f^{\prime}}=-\frac{y^{\prime}}{b-a} \quad \theta^{\prime}=-\frac{y^{\prime}}{b}
$$

On en déduit l'expression de la puissance :

$$
P=-\frac{1}{f^{\prime}}\left(1-\frac{a}{b}\right)=P_{i}\left(1-\frac{a}{b}\right)
$$

On trouve donc que la puissance dépend en général de la position de l'œil et du réglage de l'instrument. Elle est égale à la puissance intrinsèque $P_{i}$ dans deux cas

- l'image est à l'infini $(b=\infty)$ : on retrouve le cas du réglage précédent

- l'œil est placé au foyer image de l'instrument ( $a=0)$ : la taille de l'image $\theta$ ' est alors indépendante du réglage de l'instrument.

On aura donc souvent intérêt à faire en sorte que l'utilisateur place son œil au foyer image de l'instrument.

La puissance s'exprimera en dioptries $\left(\delta \mathrm{ou} \mathrm{m}^{-1}\right)$. On donnera souvent la valeur absolue de la puissance et on précisera par ailleurs si le système est convergent (par exemple une lentille plan convexe de focale $100 \mathrm{~mm}$ est un système convergent de puissance 10 dioptries).

Pour un système de type microscope composé d'un objectif (de courte focale) et d'un oculaire, la puissance du système peut s'exprimer en fonction des caractéristiques de ses éléments sous la forme :

$$
\begin{gathered}
P_{\text {microscope }}=\frac{\theta^{\prime}}{y}=\frac{\theta^{\prime}}{y_{\text {int }}} \cdot \frac{y_{\text {int }}}{y} \\
P_{\text {microscope }}=P_{\text {oculare }} \cdot\left(g_{y}\right)_{\text {objectif }}
\end{gathered}
$$

\subsubsection{Grossissement}

C'est un paramètre qui caractérise naturellement les instruments oculaires destinés à l'observation d'objets éloignés. Nous verrons qu'on peut également l'étendre au cas d'instruments oculaires du type microscope, visant des objets proches.

Le grossissement est défini comme le rapport entre l'angle $\theta$ ' sous lequel est vue (par l'œil) l'image formée par l'instrument et l'angle $\theta$ sous lequel est vu l'objet à l'œil nu.

$$
G=\frac{\theta^{\prime}}{\theta}
$$

5.3.3.1 Cas d'un objet et d'une image à l'infini. L'instrument est alors réglé afocal, ce qui est confortable pour l'œil normal. Les angles $\theta$ et $\theta$ ' sont alors les diamètres apparents de l'objet et de l'image. Le grossissement est simplement le grandissement angulaire du système afocal. Il 
est indépendant de la position de l'œil de l'observateur : c'est le grossissement intrinsèque $\boldsymbol{G}_{\boldsymbol{i}}$.

Son expression peut être déterminée en décomposant le système afocal en deux soussystèmes focaux, appelés généralement objectif et oculaire, placés de telle sorte que le foyer image de l'objectif soit confondu avec le foyer objet de l'oculaire.

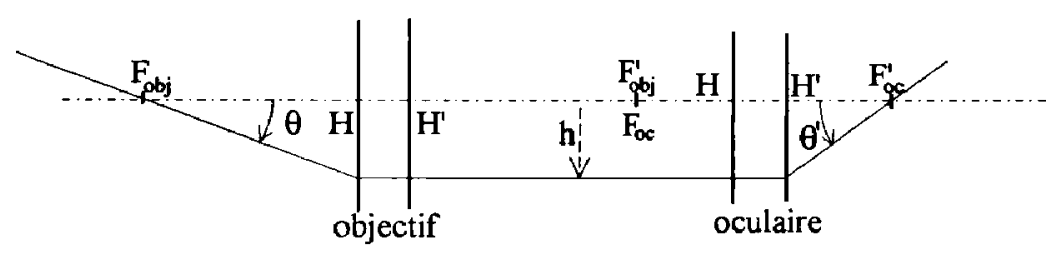

On détermine facilement le grossissement intrinsèque à partir de la construction d'un rayon issu de l'objet à l'infini et passant par $\mathrm{F}_{\mathrm{obj}}$. On obtient ainsi :

$$
G_{i}=\frac{f_{\text {objectif }}}{f_{\text {oculaire }}^{\prime}}
$$

5.3.3.2 L'objet est à l'infini, l'image est à distance finie. Il s'agit d'un instrument oculaire, l'image doit être entre le ponctum remotum et le ponctum proximum de l'œil. Dans ce cas l'angle $\theta$ ' va dépendre de la position de l'œil et du réglage de l'instrument. On peut exprimer le grossissement à partir de la construction suivante :

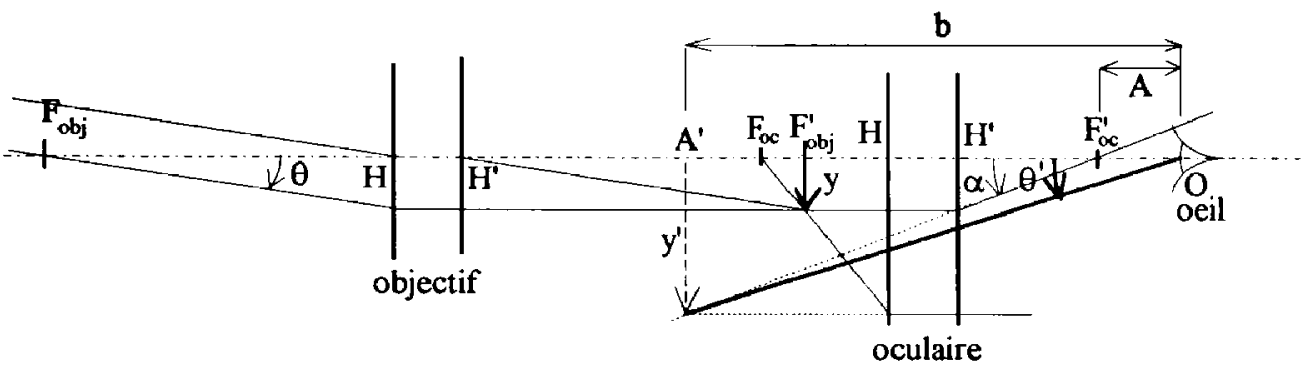

La figure ressemble à celle que l'on avait faite pour déterminer la puissance dans le cas d'une image à distance finie, sauf qu'ici on note A la distance du foyer image de l'oculaire (et non du système total) à l'œil : $A=\overline{F^{\prime} O}$. On peut par exemple écrire le grossissement du système afocal à partir de la puissance de l'oculaire :

$$
G=\frac{\theta^{\prime}}{\theta}=\frac{\theta^{\prime}}{y} \cdot \frac{y}{\theta}=-P_{o c} \cdot f_{o b j}
$$


En utilisant l'expression de la puissance de l'oculaire réglé pour une image à distance finie, on obtient :

$$
G=\frac{f_{o b j}}{f_{\alpha c}^{\prime}}\left(1-\frac{A}{b}\right)=G_{1}\left(1-\frac{A}{b}\right)
$$

On retrouve bien le grossissement intrinsèque lorsque l'instrument est réglé en afocal $(b=\infty)$, mais aussi lorsque l'ceil est placé dans le plan focal image de l'oculaire. On aura intérêt à placer l'œil de cette façon pour que la grandeur de l'image ne dépende pas du réglage de l'instrument.

5.3.3.3 Grossissement dans le cas d'un objet à distance finie. Bien que la puissance soit le paramètre le plus naturel dans le cas d'un instrument oculaire visant un objet rapproché (type microscope), on peut également définir un grossissement pour un tel instrument. Dans ce cas, l'angle $\theta$ sous lequel est vu l'objet à l'œil nu va dépendre des propriétés de l'œil. Il est logique de prendre pour $\theta$ la valeur maximale obtenue dans le cas où l'objet est au ponctum proximum, distant de $d$ de l'œil. On aura donc

$$
\theta=\frac{y}{d}
$$

Quant à $\theta^{\prime}$, on peut reprendre son expression à partir de celle de la puissance dans le cas d'un réglage quelconque de l'instrument (avec les notations du $2 \mathrm{~b}$ ) :

$$
\theta^{\prime}=P \cdot y=-\frac{y}{f^{\prime}}\left(1-\frac{a}{b}\right)
$$

L'expression du grossissement est :

$$
G=\frac{\theta^{\prime}}{\theta}=-\frac{d}{f^{\prime}}\left(1-\frac{a}{b}\right)
$$

On définit alors un grossissement intrinsèque $(a=0$ ou $b=\infty)$ commercial en prenant un œil standard dont le ponctum proximum $d$ est à $25 \mathrm{~cm}$ :

$$
G_{i c}=-\frac{250 \mathrm{~mm}}{f^{\prime}(\text { en } \mathrm{mm})}=\frac{P_{i}(\text { en dioptries })}{4}
$$

Par exemple un oculaire dit $4 \mathbf{x} 10$ » est un oculaire de grossissement commercial intrinsèque 10 : sa puissance intrinsèque est alors de 40 dioptries et sa focale de $25 \mathrm{~mm}$.

Pour un microscope, on parlera aussi de grossissement :

$$
\begin{gathered}
G_{\text {microscope }}=\frac{P_{\text {microscope }}}{4}=\frac{P_{\text {oqulaire }}}{4} \cdot\left(g_{y}\right)_{\text {objectif }} \\
G_{\text {micrascope }}=G_{\text {oculaire }} \cdot\left(g_{y}\right)_{\text {objectif }}
\end{gathered}
$$

Ainsi si on associe un objectif de grandissement 20 et un oculaire $\mathbf{x} 10$, on obtient un viseur (ou un microscope) de grossissement 200, de puissance $800 \delta$ et de focale $1.25 \mathrm{~mm}$. 


\section{PROPRIÉTÉS DES INSTRUMENTS : CHAMPS EN LARGEUR ET EN PROFONDEUR}

Nous avons dit dans la partie précédente que le champ d'un instrument est la portion d'espace qui est vue à travers l'instrument. Nous allons être plus précis maintenant sur ce qui limite cette portion d'espace et ceci va nous amener à distinguer :

- le champ en largeur, section du champ orthogonale à l'axe de l'instrument, qui va être déterminé par les différents diaphragmes à l'intérieur de l'instrument (dimensions finies des lentilles et des miroirs, diaphragmes à iris), voire dans le plan de l'image elle-même (format du film, taille du détecteur);

- le champ en profondeur, section du champ le long de l'axe, qui fait intervenir la netteté de l'image et dépend du détecteur utilisé.

Les champs en largeur et en profondeur ne seront bien définis que pour un instrument dont la fonction est bien déterminée (en particulier la position de l'objet observé), dont les diaphragmes sont fixés, et dont le détecteur est précisé.

\subsection{Champ en largeur}

Il s'agit désormais de prendre en compte les dimensions limitées des éléments optiques (ou des diaphragmes mécaniques) formant un instrument, pour déterminer les rayons issus du plan objet visé pouvant effectivement traverser l'instrument. Ceci nous conduira à définir les notions d'ouverture et de champ en largeur. Nous nous limiterons ici au cas de systèmes centrés, que nous supposerons stigmatiques et aplanétiques pour la conjugaison utilisée (ceci implique que les aberrations soient ensuite corrigées pour l'ouverture et le champ que l'on aura déterminés).

Nous avons choisi ici d'aborder le problème sur un exemple, puis de généraliser la méthode intuitive à un système quelconque, à deux diaphragmes ou plus.

\subsection{Etude d'un exemple}

On considère un viseur constitué de deux lentilles simples

- un objectif de grandissement $g_{y}=-2$, de focale $f_{1}=40 \mathrm{~mm}$ et de diamètre $\emptyset_{1}=6 \mathrm{~mm}$;

- un oculaire $\times 12.5$ de diamètre $\emptyset_{2}=4 \mathrm{~mm}$.

Remarque : un tel viseur a une focale négative (ici $-10 \mathrm{~mm}$ ), de même que la plupart des microscopes.

Le viseur étant réglé à l'infini, on a la configuration suivante :

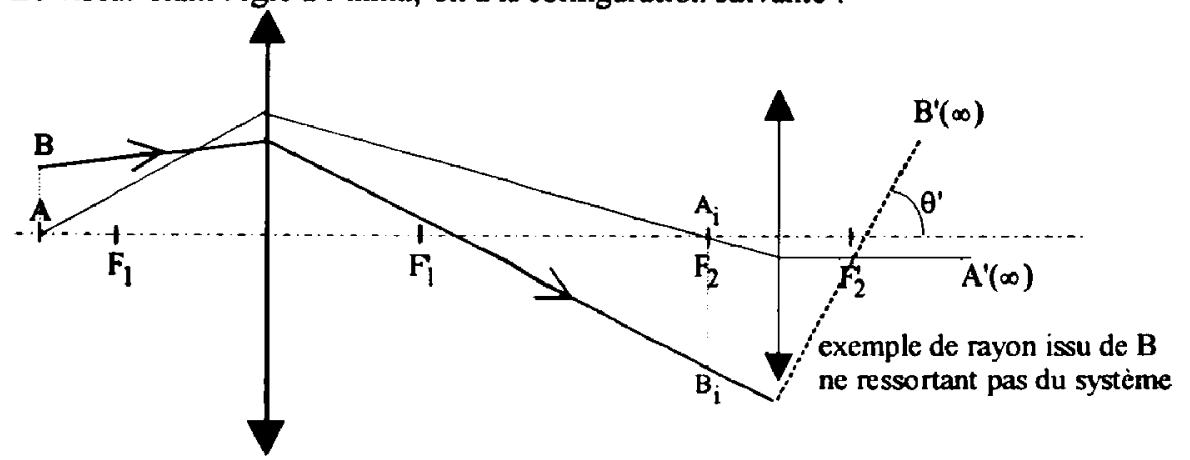


On a montré sur ce schéma l'exemple d'un rayon issu d'un point objet $B$ dans le champ, et qui est arrêté par la monture de l'oculaire.

Déterminons tout d'abord les limites d'un faisceau de rayons issu du point objet $\mathrm{A}$ sur l'axe et pouvant ressortir du système. Graphiquement, on trouve facilement ce faisceau :

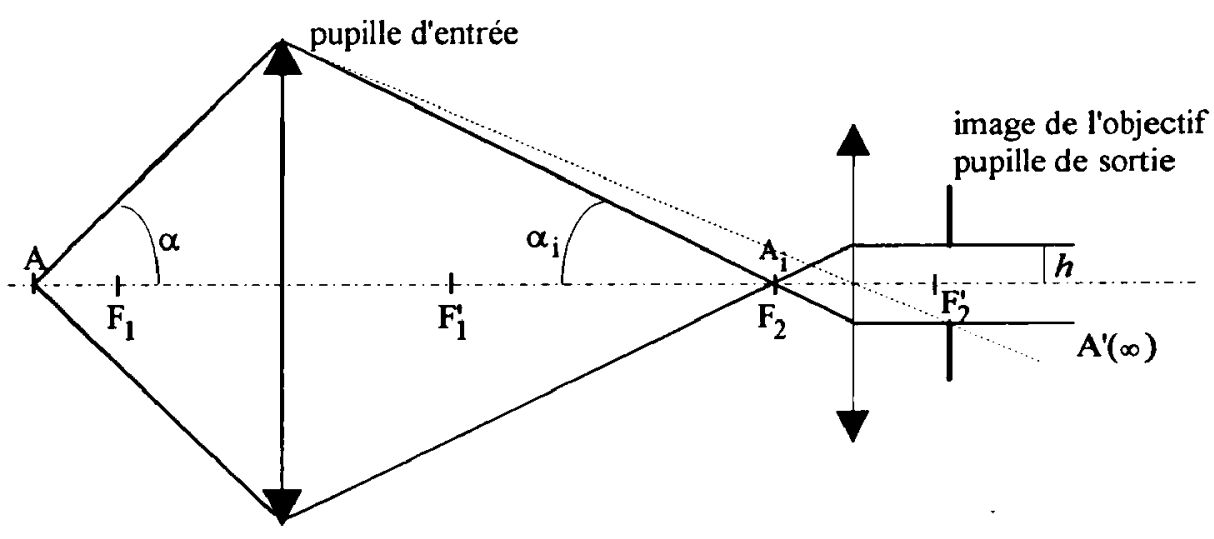

On a ainsi déterminé l'angle d'ouverture $\alpha$ du viseur dans l'espace objet : $\alpha=\frac{\varnothing_{1}}{2 \overline{O_{1} \mathrm{~A}}}=0.05 \mathrm{rad}$

Il est limité par la monture de l'objectif, qui est alors appelée pupille de l'instrument, ici même pupille d'entrée puisque cette pupille se trouve dans l'espace objet. C'est dans l'espace intermédiaire, où l'angle d'ouverture du faisceau vaut $\alpha_{i}=0.025 \mathrm{rad}$, que l'on distingue le mieux que c'est l'objectif qui limite l'ouverture, car c'est celui qui est vu sous l'angle le plus petit depuis l'image intermédiaire $A_{i}$. On peut cependant vérifier que le même raisonnement peut être appliqué dans l'espace objet ou dans l'espace image, à condition d'y conjuguer les diaphragmes. Par exemple dans l'espace image, on peut construire l'image des bords de l'objectif : on obtient un diaphragme de rayon $h=1 \mathrm{~mm}$, qui est inférieur au rayon de l'oculaire : on retrouve bien là aussi que c'est l'image de l'objectif qui est vue sous "l'angle » le plus petit depuis l'image (ici l'image étant à l'infini, l'angle est remplacé par une hauteur par rapport à l'axe). L'image de l'objectif, limitant l'ouverture dans l'espace image, est appelée pupille de sortie du viseur.

Intéressons-nous maintenant à un point objet B situé hors de l'axe. Pour un point B choisi au hasard, on peut déterminer graphiquement les limites du faisceau de rayons traversant le système : 


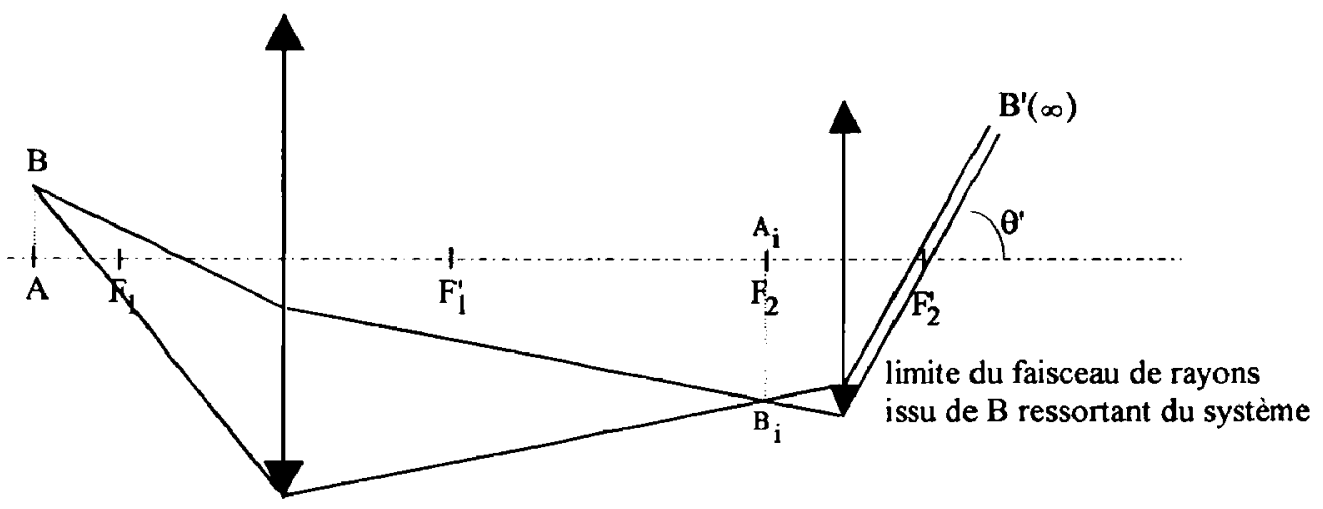

On voit que sur cet exemple le faisceau de rayons est moins ouvert que dans le cas de $\mathrm{A}$, car l'oculaire provoque également une limitation : l'oculaire est alors appelé lucarne de l'instrument. L'image du point B sera donc moins lumineuse que celle de A.

Cherchons alors à partir de quel point $\mathrm{B}$ la limitation de l'oculaire intervient. On l'obtient graphiquement :

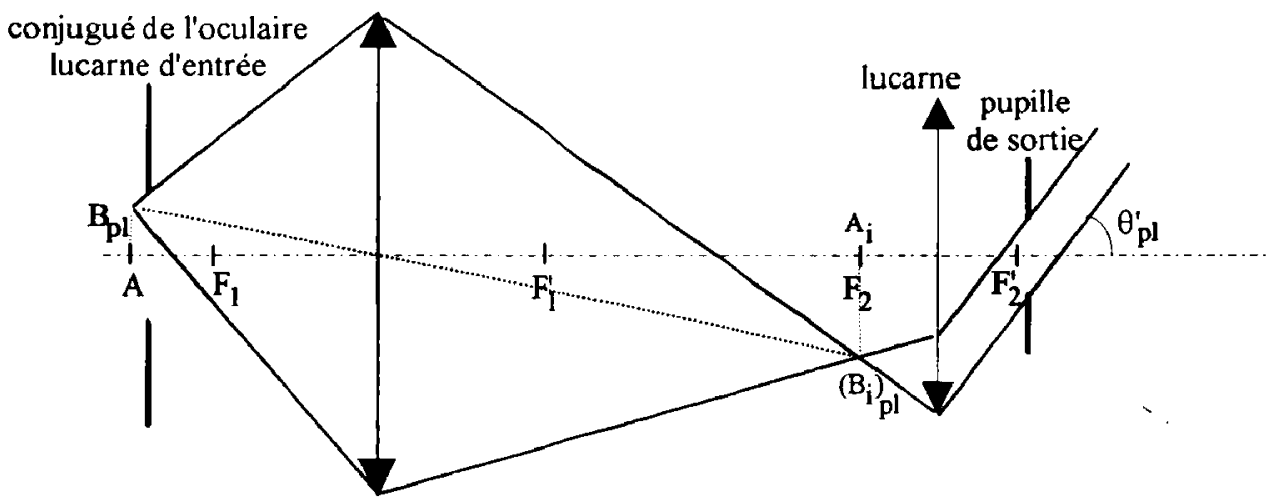

Le point $\mathrm{B}_{\mathrm{pl}}$ ainsi déterminé est appelé le bord du champ de pleine lumière. A noter que le système étant de révolution, le champ de pleine lumière est un cercle de rayon $y_{p l}=\overline{\mathrm{AB}_{\mathrm{pl}}}$. Là encore on s'aperçoit que la construction se fait le plus simplement dans l'espace intermédiaire, mais elle peut de façon complètement analogue être faite dans l'espace image ou dans l'espace objet en conjuguant objectif et oculaire dans ces espaces. Dans l'espace intermédiaire, le rayon du champ de pleine lumière $\left(y_{i}\right)_{p l}$ est donné par :

$$
\frac{3+\left(y_{i}\right)_{p l}}{120}=\frac{2-\left(y_{i}\right)_{p l}}{20} \quad \text { d'où } \quad\left(y_{i}\right)_{p l}=1.29 \mathrm{~mm}
$$


Par conjugaison, on a dans l'espace objet: $y_{p l}=0.64 \mathrm{~mm}$, et dans l'espace image : $\theta^{\prime}{ }_{p l}=0.064 \mathrm{rad}$.

Si on continue à s'éloigner de l'axe au-delà du champ de pleine lumière, l'ouverture du faisceau de rayons traversant le système diminue. On passe alors par une situation intermédiaire où environ la moitié du faisceau est obturé par rapport au cas du champ de pleine lumière :

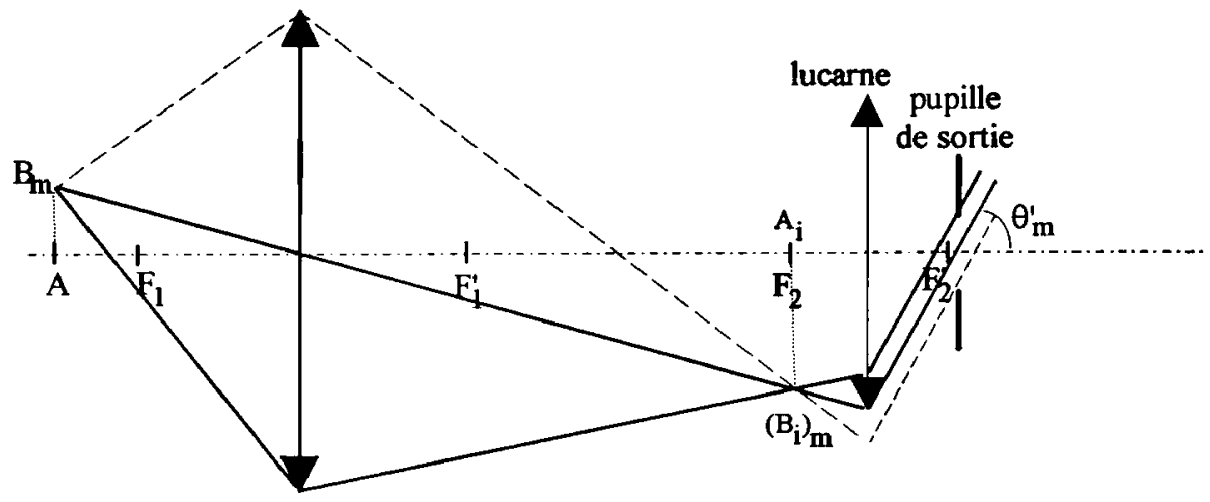

Cette position est appelée champ moyen de l'instrument. On l'a déterminé dans l'espace intermédiaire en joignant le centre de la pupille au bord de la lucarne et on a :

$$
\left(y_{i}\right)_{m}=1.71 \mathrm{~mm} \quad y_{m}=0.86 \mathrm{~mm} \quad \theta_{m}^{\prime}=0.086 \mathrm{rad}
$$

Enfin en s'éloignant encore plus de l'axe, on finit par atteindre une situation où plus aucun rayon ne traverse le système :

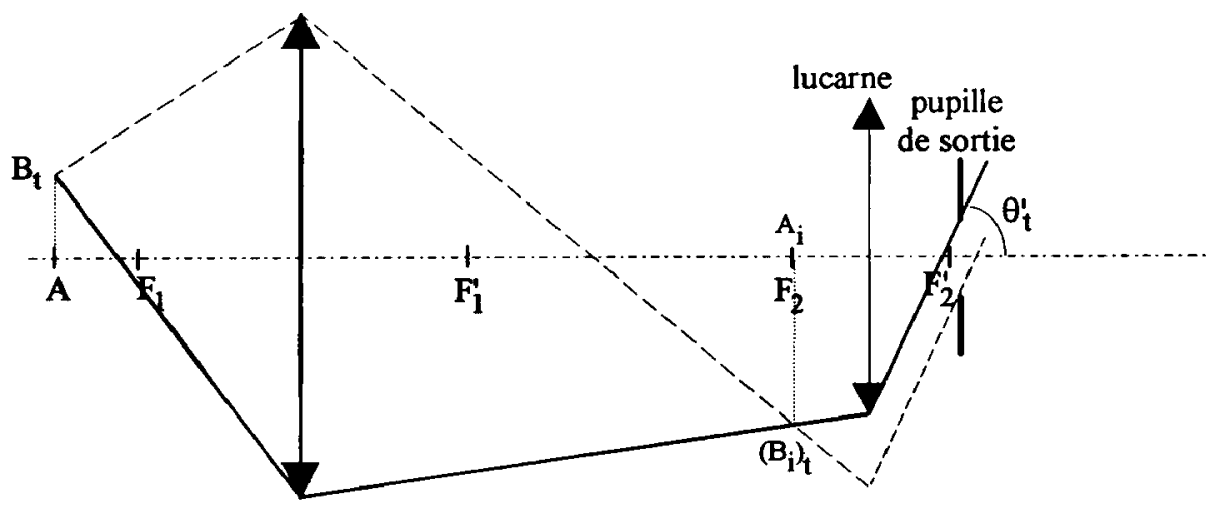

C'est le bord du champ total, au-delà duquel l'instrument ne donne plus aucune image. Son rayon vaut ici :

$$
\left(y_{i}\right)_{t}=2.14 \mathrm{~mm} \quad y_{t}=1.07 \mathrm{~mm}
$$

$\theta^{\prime}=0.107 \mathrm{rad}$ 


\subsubsection{Méthode générale}

En s'inspirant de l'exemple que nous venons d'étudier, on peut dégager une méthode générale d'analyse d'un problème de champ, pour un système ayant deux diaphragmes ou plus. On va procéder en trois étapes :

- on choisit un espace «de travail» (espace objet ou image ou l'un des espaces intermédiaires) dans lequel on détermine la position et la dimension des conjugués de tous les diaphragmes du système ainsi que la position du plan image. On a intérêt bien sûr à choisir l'espaçe de travail de façon à minimiser le nombre de conjugaisons à calculer (c'était l'espace intermédiaire dans le cas de l'exemple).

- on résout le problème dans cet espace, c'est-à-dire qu'on y détermine l'ouverture et le champ : on cherche les rayons qui passent tout juste à travers tous les diaphragmes, soit issus du point image sur l'axe (détermination de l'ouverture), soit d'un point hors d'axe (détermination du champ). Nous verrons plus en détail dans les deux paragraphes suivants comment trouver pratiquement ces rayons limites.

- on sait par conjugaison que les rayons qui n'étaient pas obturés dans l'espace de travail ne le seront dans aucun des autres espaces. Il suffit alors de ramener par conjugaison l'ouverture et le champ dans l'espace objet et dans l'espace image On a intérêt alors à vérifier les résultats trouvés en faisant des tracés de rayons à travers le système

Remarque : dans toute la suite, on utilisera le terme d'image au sens de-conjugué, indépendamment du sens réel de propagation de la lumière. Il faudra donc faire attention au sens d'utilisation des formules de conjugaison, et à bien identifier l'espace dans lequel se trouve cette image dans le cas ou elle est virtuelle.

\subsubsection{Ouverture d'un instrument. Pupille.}

$\mathrm{Si}$ on généralise la méthode utilisée dans l'exemple du viseur, on voit qu'on détermine la pupille en cherchant le diaphragme (réel ou conjugué d'un diaphragme réel), qui est vu sous l'angle le plus petit depuis le point image sur l'axe :

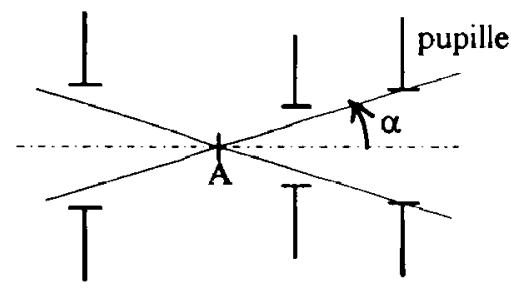

On obtient l'angle d'ouverture $\alpha$ du système dans cet espace, puis par conjugaison l'angle d'ouverture objet et la pupille d'entrée du système ainsi que l'angle d'ouverture image et la pupille de sortie.

Signalons ici une convention relative à l'ouverture des objectifs (par exemple les objectifs photographiques) que l'on caractérise par un nombre d'ouverture $\mathbf{N}$, parfois appelé nombre d'ouverture géométrique. Ce nombre représente le rapport entre la focale de l'objectif et le diamètre de sa pupille d'entrée : on dit alors que l'objectif est ouvert à $f / \mathrm{N}$ (attention, ceci n'est pas égal à l'angle $\alpha$ puisque la distance objet-pupille d'entrée n'est pas égale à $f^{\prime}$ ). On retrouvera ce paramètre dans la partie concernant la photométrie $(\S 7)$ 


\subsubsection{Champs d'un instrument. Lucarnes.}

6.1.4.1 Cas d'un système à deux diaphragmes. C'est le cas de l'exemple du viseur traité plus haut. L'un des diaphragmes ayant été identifié comme la pupille, c'est forcément l'autre qui limitera le champ et sera done la lucarne ou diaphragme de champ. De façon analogue à ce qu'on a vu dans l'exemple du viseur, on détermine les limites du champ de pleine lumière, du champ moyen et du champ total. Dans l'espace de travail où on a ramené les conjugués des deux diaphragmes et le plan image, on aura ainsi :
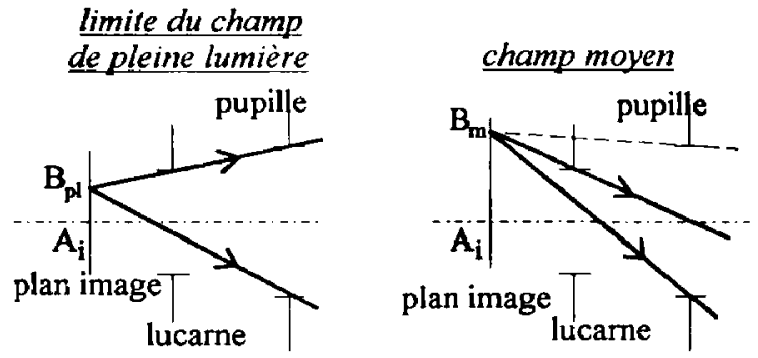

limite du champ total

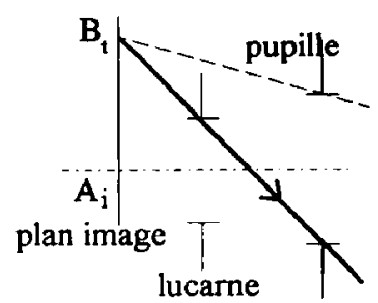

On a choisi ici un cas où les positions relatives des diaphragmes et du plan image sont différentes de celles du viseur.

Pour illustrer la transition entre les différentes zones, il est intéressant de représenter dans le plan de la lucarne la trace du cône de rayons issus de B s'appuyant sur le contour de la pupille d'entrée :

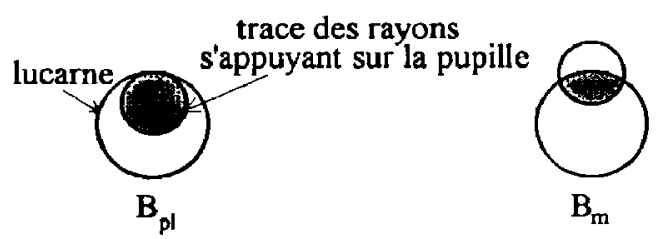

limite du champ de pleine lumière champ moyen

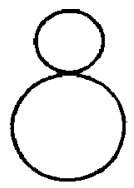

$\mathbf{B}_{\mathbf{t}}$

limite du champ total

On peut faire grâce à ce schéma une petite incursion dans le domaine de la photométrie On voit en effet que la quantité de lumière transmise va être quasiment constante dans la zone de champ de pleine lumière (on verra qu'elle ne l'est pas rigoureusement), puis qu'elle va chuter progressivement pour s'annuler au bord du champ total. Cette zone intermédiaire est encore appelée champ de contour. On comprend qu'en général, on souhaite un champ de pleine lumière le plus grand possible; de plus on préférera souvent supprimer le champ de contour par une méthode que l'on verra un peu plus loin.

Remarque : il peut se produire que les deux diaphragmes soient vus depuis le point objet sur l'axe A sous le même angle. Il n'y a alors pas de champ de pleine lumière, ce qui est peu souhaitable. 
6.1.4.2 Cas d'un instrument à plus de deux diaphragmes. Lorsque le système a plus de deux diaphragmes, le problème est plus complexe car il peut arriver que plusieurs diaphragmes interviennent dans la limitation du champ. Un schéma permet de déterminer les champs dans ce cas. Pour donner une idée des situations possibles, nous avons représenté ci-dessous deux exemples :

exemple I
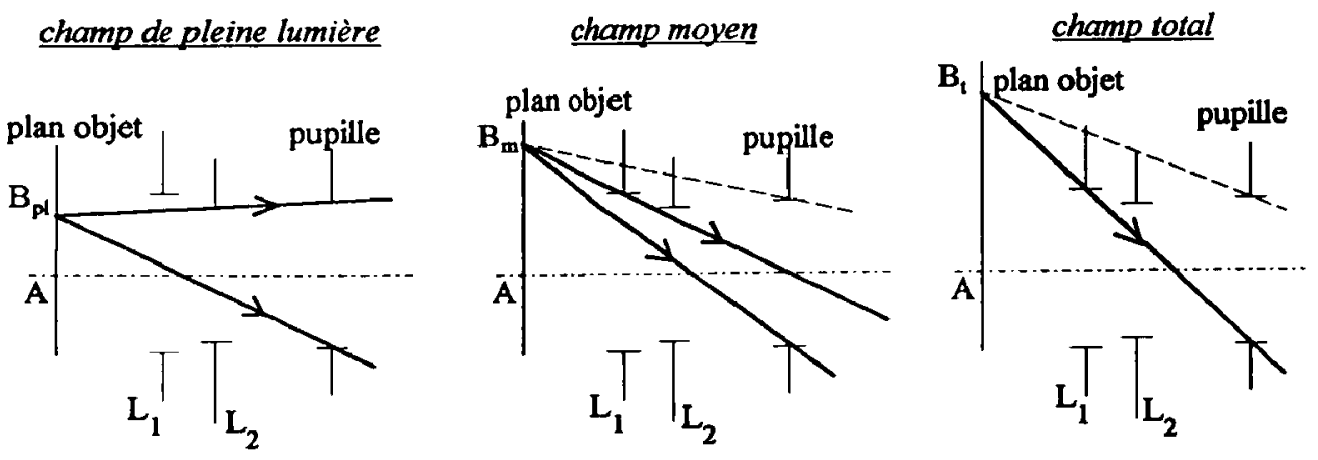

Dans ce premier exemple, le champ de pleine lumière est limité par la lucarne $\mathrm{L}_{2}$, tandis que le champ moyen et le champ total sont limités par l'autre lucarne $\mathrm{L}_{\mathbf{1}}$.

exemple 2

champ de pleine lumière

champ total
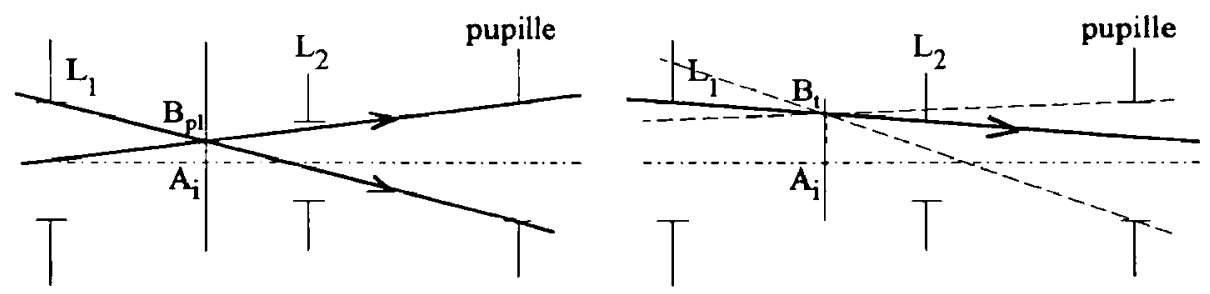

Ici, le champ de pleine lumière est limité par $L_{1}$, puis $L_{2}$ va commencer à obturer l'autre côté du faisceau, de sorte qu'il n'apparait pas clairement de champ moyen. Le champ total est finalement limité par les deux bords de $\mathrm{L}_{1}$ et $\mathrm{L}_{2}$ et ne fait pas intervenir le diamètre de la pupille.

\section{I.5 Diaphragmation du champ de contour}

Quand on sort du champ de pleine lumière, l'éclairement dans le plan de l'image chute progressivement jusqu'à s'annuler au bord du champ total. On appelle champ de contour cette zone entre champ de pleine lumière et champ total. 
On souhaite très souvent supprimer ce champ de contour de façon à avoir une coupure nette d'éclairement au bord du champ de pleine lumière. Par exemple dans le cas d'un appareil photo, on vient placer un diaphragme de champ supplémentaire (cache $24 \times 36$ par ex) dans le plan de l'image finale. On peut également placer ce diaphragme dans un espace intermédiaire, à condition que l'image dans cet espace soit réelle.

$\mathrm{Si}$ on reprend le cas du viseur précédent, on va diaphragmer le champ de contour dans l'espace intermédiaire, le plan objet n'étant pas accessible en général et l'image étant à l'infini :

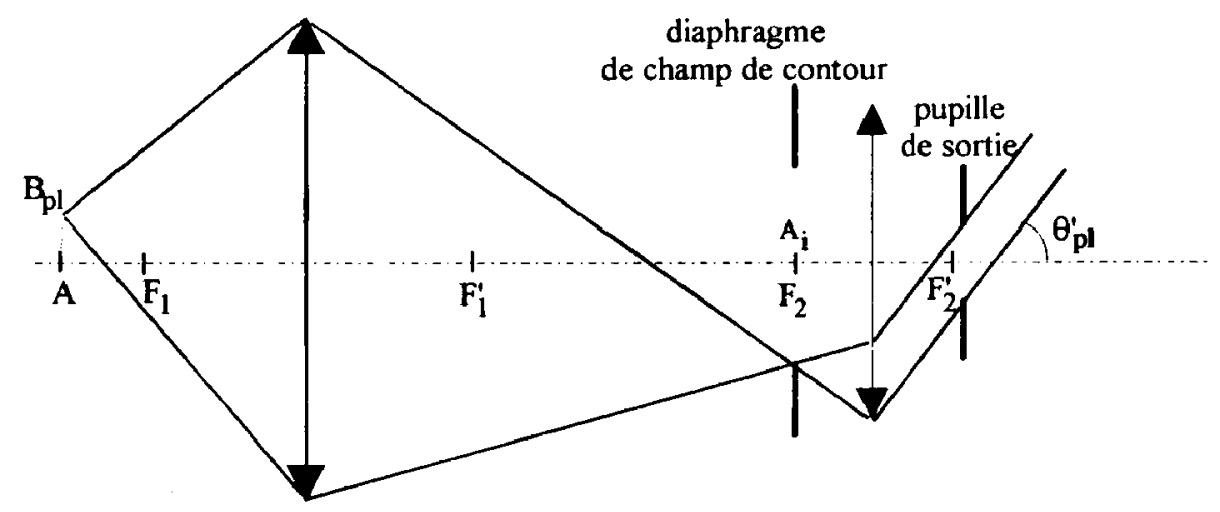

\subsubsection{Cercle oculaire. Position de l'ail derrière un instrument oculaire.}

On appelle cercle oculaire, la pupille de sortie d'un instrument destiné à l'observation visuelle. C'est en effet à cet endroit qu'on a intérêt à placer la pupille d'œil. Si on la place ailleurs, elle va devoir être prise en compte comme diaphragme supplémentaire dans le système et risque de réduire le champ de pleine lumière. En fait, en plaçant l'œil sur le cercle oculaire et en choisissant une pupille de sortie de l'instrument plus petite que la pupille d'oil (qui est d'environ $2 \mathrm{~mm}$ en vision de jour), les champs en largeur de l'instrument ne seront pas modifiés par l'œil.

Remarquons que ceci n'est possible qu'à condition que la pupille de sortie de l'instrument soit réelle (voire pas trop proche de la dernière lentille de l'oculaire). Un exemple d'instrument oculaire à pupille de sortie virtuelle est la lunette de Galilée :

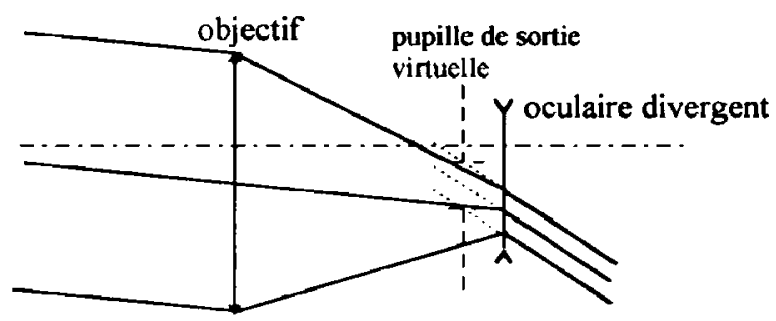

\subsubsection{Rôle du verre de champ dans un oculaire}

Les instruments visuels sont souvent constitués d'un objectif et d'un oculaire. Les instruments de type microscope, comme le viseur que nous avons utilisé comme exemple, sont constitués 
d'un objectif qui forme une image intermédiaire très agrandie d'un objet à distance finie (proche), image qui est reprise par l'oculaire qui la rend observable à l'œil, à l'infini (image intermédiaire placée au foyer objet de l'oculaire). Dans le cas d'un instrument visuel de type lunette astronomique, les foyers image de l'objectif et objet de l'oculaire sont confondus de façon à former un système afocal.

Nous allons voir, sur notre exemple de viseur, l'intérêt du point de vue des champs d'utiliser un oculaire à deux lentilles, la plus proche de l'œil étant appelée verre d'ceil et l'autre verre de champ.

Rappelons tout d'abord les propriétés de déviation d'un rayon par une lentille mince convergente :

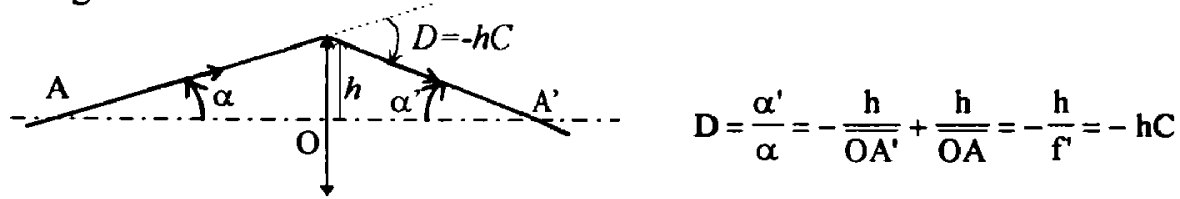

On voit qu'une lentille convergente ramène toujours les rayons vers l'axe

Reprenons le schéma du champ moyen de notre viseur :

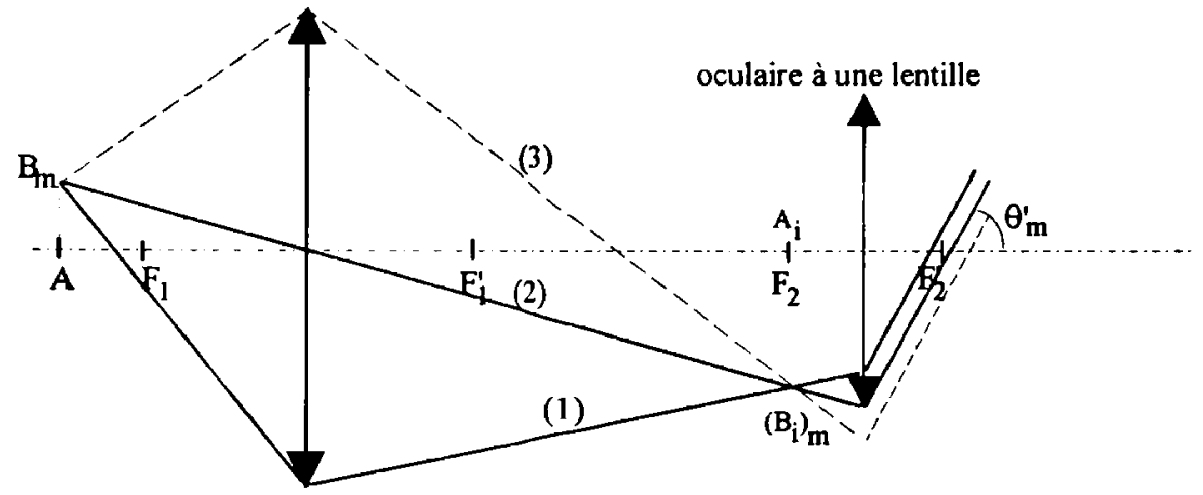

Plaçons maintenant une lentille convergente dans le plan de l'image intermédiaire :

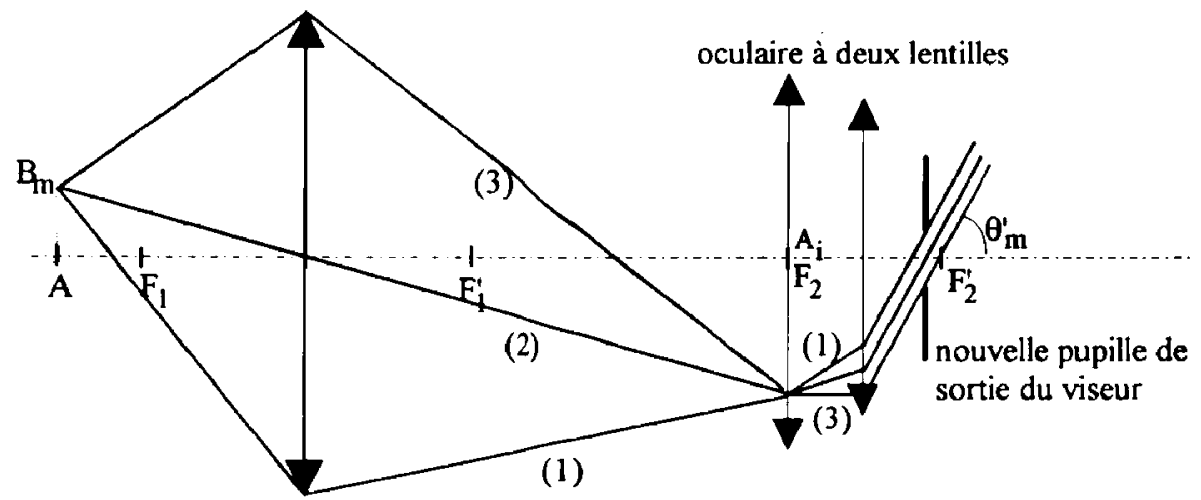


Puisqu'elle est dans le plan de l'image intermédiaire, la lentille supplémentaire ne change rien à la position ni à la grandeur de l'image, qui est toujours à l'infini de rayon angulaire $\theta^{\prime}{ }_{m}$. Par contre, le champ a augmenté puisque le rayon (3), obturé précédemment, peut ainsi être ramené vers l'axe de façon à passer à travers la deuxième lentille de l'oculaire.

On voit ainsi qualitativement que la lentille supplémentaire permet d'augmenter le champ de la lunette : c'est le verre de champ de l'oculaire, tandis que la lentille la plus proche de l'œil est appelée verre d'œil.

Grâce au raisonnement qualitatif précédent et à l'expression de la déviation de la lentille $\mathrm{L}_{2}$ $(D=-h C)$, on voit que plus le verre de champ est convergent (à diamètre égal), plus le champ sera augmenté. Toutefois ceci va rapprocher la pupille de sortie de l'instrument du verre d'cil (cf figure) : lorsque la focale du verre de champ est égale à celle du verre d'œil, le foyer image F' du viseur est sur le verre d'oil, et la pupille de sortie est très proche de F'. Elle va ensuite devenir virtuelle et on ne pourra plus y placer la pupille de l'œil. Ceci fixe donc une limite à l'augmentation du champ par le verre de champ.

En pratique, il n'est pas souhaitable de placer une lentille dans le plan de l'image intermédiaire, d'une part parce qu'on ne peut pas y mettre un réticule, d'autre part parce que toute poussière ou rayure sur la surface de la lentille sera vue nette en même temps que l'image. On conserve alors le principe d'un oculaire à deux lentilles mais on décale légèrement le verre de champ par rapport au plan focal objet du verre d'œil. Les oculaires les plus souvent utilisés sont l'oculaire de Ramsden 3-2-3, dont le foyer objet est réel (oculaire positif) légèrement en avant du verre de champ, et l'oculaire d'Huyghens 3-2-1, dont le foyer objet est virtuel (oculaire négatif) situé entre les deux lentilles.

Remarque : dans le cas d'un oculaire négatif type Huyghens, le foyer objet, virtuel, n'est pas accessible, il faut donc placer le réticule éventuel dans le plan conjugué par le verre de champ, à savoir au foyer objet du verre d'ail.

\subsection{Champ en profondeur}

La notion de profondeur de champ est bien connue des photographes : c'est la distance, le long de l'axe de visée, sur laquelle l'image reste nette. On sait, par expérience, que sur un appareil photo, cette profondeur de champ augmente quand on ferme le diaphragme. Elle dépend également du type de film utilisé, plus précisément du grain de l'émulsion (d'autant plus gros que le film est rapide)

De façon générale, la profondeur de champ est caractéristique d'une association instrument - détecteur.

\subsection{Cas des instruments de projection}

Continuons sur l'exemple de l'appareil photo, que nous supposerons dépourvu d'aberrations et d'ouverture suffisamment grande pour que la tache de diffraction soit négligeable devant le diamètre moyen $g$ du grain de l'émulsion utilisée.

Un objet ponctuel $A$ donne à travers l'objectif photo une image ponctuelle $A^{\prime}$. Mais, à cause du détecteur, on ne peut la distinguer d'une image de taille $\mathrm{g}$. Ceci implique dans l'espace image une certaine tolérance sur la position du détecteur, que l'on appelle la profondeur de foyer 


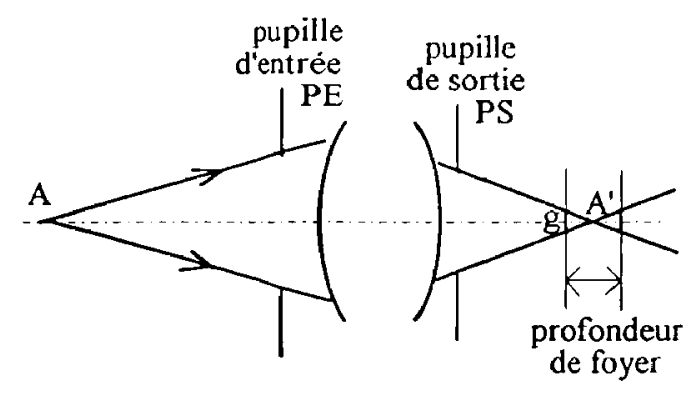

Dans l'espace objet, pour une position fixée du détecteur, on aura également une certaine tolérance sur la position de l'objet A pour que son image ne couvre qu'un grain du détecteur ; c'est la profondeur de champ :

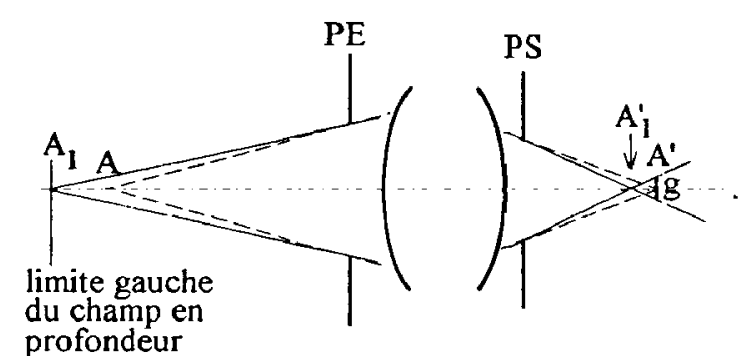

6.2.1.1 Calcul de la profondeur de foyer

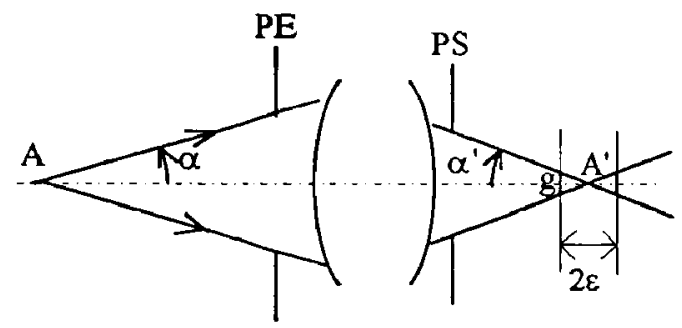

La profondeur de foyer est symétrique par rapport à la position de l'image A'. Si on note $\alpha$ ' l'angle d'ouverture dans l'espace image, on peut écrire :

$$
2 \varepsilon=g / \alpha^{\prime}
$$

La profondeur de foyer est d'autant plus grande que l'ouverture de l'instrument est faible. Pour assurer une mise au point correcte du détecteur par rapport au plan image, on aura intérêt à se placer à l'ouverture maximale. 
Notons enfin que la netteté de l'image ne chute pas brutalement à $\pm \varepsilon$ mais diminue progressivement : la profondeur de foyer $2 \varepsilon$ donne une échelle caractéristique de cet effet.

\subsubsection{Calcul de la profondeur de champ}

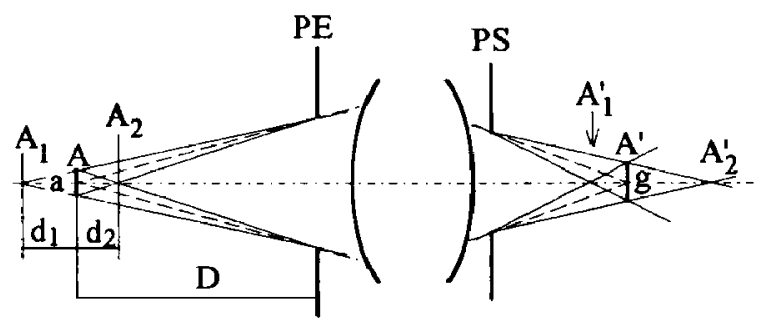

Le détecteur est maintenant fixe dans le plan de l'image $A^{\prime}$, et on cherche les positions extrêmes $A_{1}$ et $A_{2}$ dans l'espace objet pour lesquelles la tache image dans le plan du détecteur a un diamètre $\mathrm{g}$. Elles correspondent donc à une « tache objet » dans le plan de l'objet $\mathrm{A}$ de diamètre :

$$
a=g / g_{y}
$$

où $g_{y}$ est le grandissement transversal de l'instrument pour les points conjugués $A$ et $A$ '.

Notons $\varnothing$ le diamètre de la pupille d'entrée et $\mathrm{D}$ la distance de l'objet $\mathrm{A}$ à la pupille d'entrée. On a :

$$
\begin{aligned}
& \frac{d_{1}}{D+d_{1}}=\frac{a}{\varnothing} \quad \mathrm{d}^{\prime} \text { où: } \quad d_{1}=\frac{a D}{\varnothing-a} \\
& \frac{d_{2}}{D-d_{2}}=\frac{a}{\varnothing} \quad \text { d'où: } \quad d_{2}=\frac{a D}{\varnothing+a}
\end{aligned}
$$

Remarquons que la profondeur de champ n'est pas symétrique par rapport au plan de mise au point $\left(d_{1}>d_{2}\right)$. Ainsi lorsqu'on photographie un défilé par exemple, on a intérêt à effectuer la mise au point en gros au premier tiers de la colonne. On peut en fait être plus précis et calculer la distance $\mathrm{D}$ de meilleure mise au point connaissant les deux positions extrêmes $D_{1}$ et $D_{2}$ de la colonne (on suppose ici que l'ouverture a été choisie pour que la profondeur de champ soit suffisante). On montre que :

$$
D=\frac{2 D_{1} D_{2}}{D_{1}+D_{2}}
$$

Par exemple pour un champ en profondeur compris entre 3 et 6 mètres on a intérêt à faire la mise au point à 4 mètres ; ou pour un champ compris entre 1 et 3 mètres, on met au point à $1.5 \mathrm{~m}$ (on n'est pas loin de la règle approximative du premier tiers)

Il est également intéressant de déterminer quelle ouverture il faut choisir pour que la profondeur de champ soit suffisante pour voir toute la colonne nette. On cherche donc à relier le nombre d'ouverture $\mathrm{N}=f / \varnothing$, où $f$ est la focale de l'objectif photo, à l'extension $d$ du 
champ en profondeur autour de la distance de mise au point $D$. En faisant quelques approximations (distance $\mathrm{D}$ grande devant la focale du système et diamètre $a$ petit devant la dimension de la pupille d'entrée), on obtient la relation suivante:

$$
N=\frac{d f^{2}}{2 g D^{2}}
$$

Par exemple, avec un objectif photo standard de focale $50 \mathrm{~mm}$, en prenant comme grain $\mathrm{g}=30 \mu \mathrm{m}$, il faut une ouverture $\mathrm{N} \approx 8$ pour obtenir une profondeur de champ entre 3 et $6 \mathrm{~m}$ (mise au point à $\mathrm{D}=4 \mathrm{~m}$ ). Cette caractéristique est souvent indiquée sur les montures des appareils :

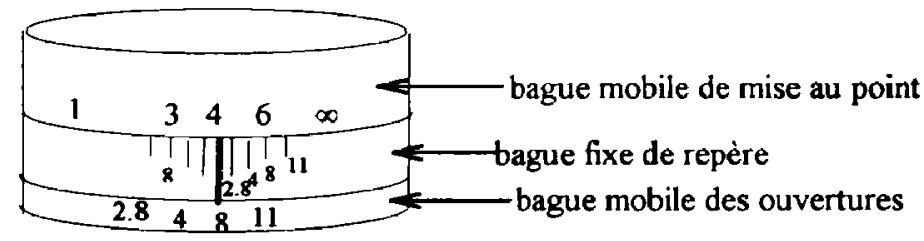

Retenons en tout cas que la profondeur de champ augmente avec le nombre d'ouverture et la distance de mise au point, et diminue avec la focale.

Remarque : dans le cas d'un instrument travaillant pour un objet à l'infini, sewl le point $A_{2}$ est défini. La profondeur de champ est alors caractérisée par la distance de $A_{2}$ à l'instrument, appelée distance hyperfocale.

\subsubsection{Cas des instruments visuels}

Dans le cas d'un instrument associé à l'œil, deux facteurs entrent en compte dans la profondeur de champ

- pour une accommodation fixée de l'œil, l'ensemble instrument + œil + rétine constitue un système de projection pour lequel on peut définir une profondeur de champ lié à la taille des cellules rétiniennes (ou à la tache de diffraction si c'est elle qui domine);

- en fait l'cil accommode quasiment automatiquement : il voit net tout objet situé sur son parcours d'accommodation. On définit alors une profondeur d'accommodation qui correspond à la zone objet de l'instrument dont l'image correspond au parcours d'accommodation de l'œil.

La profondeur de champ de l'instrument visuel est la somme de ces deux contributions, qui est surtout dominée par la profondeur d'accommodation dans le cas d'un œil normal (non presbyte).

Calcul de la profondeur d'accommodation

Prenons le cas d'un instrument du genre microscope ou viseur, destiné à l'observation d'un objet rapproché. On place l'œil dans le plan focal image de l'instrument (on a vu l'intérêt de cette situation du point de vue de la puissance, égale à la puissance intrinsèque quel que soit le 
réglage, ainsi que du point de vue des champs, la pupille de sortie de l'instrument étant souvent située au voisinage du plan focal image).

Notons $A_{P}^{\prime}$ et $A^{\prime}{ }_{R}$ les ponctum proximum et remotum de l'œil. On cherche les points $A_{P}$ et $A_{R}$ correspondants dans l'espace objet de l'instrument.

$$
\begin{array}{rlrl}
\overline{\mathrm{FA}_{\mathrm{P}}} \cdot \overline{\mathrm{F}^{\prime} \mathrm{A}_{\mathrm{P}}^{\prime}} & =-f^{2} & \overline{\mathrm{FA}_{\mathrm{R}}} \cdot \overline{\mathrm{F}^{\prime} \mathrm{A}_{\mathrm{R}}^{\prime}}=-f^{2} \\
\overline{\mathrm{FA}_{\mathrm{P}}} & =-\frac{f^{2}}{d} & \overline{\mathrm{FA}_{\mathrm{R}}} & =-\frac{f^{2}}{D}
\end{array}
$$

où $d$ et $D$ sont les distances minimale et maximale de vision distincte.

On en déduit l'expression de la profondeur d'accommodation :

$$
l=\overline{\mathbf{A}_{\mathrm{P}} \mathbf{A}_{\mathrm{R}}}=f^{2}\left(\frac{1}{d}-\frac{1}{D}\right)=f^{2} A
$$

où $A$ est l'amplitude dioptrique d'accommodation (4 dioptries pour l'œil standard). Par exemple pour un viseur de puissance 400 dioptries (focale $2.5 \mathrm{~mm}$ ), la profondeur d'accommodation vaut $25 \mu \mathrm{m}$.

Remarquons que la profondeur d'accommodation constitue l'une des causes d'incertitude de pointé longitudinal de l'instrument. Pour un objet observé fixe, l'œil qui accommode en obtient une image nette sur une plage $l$ de position du viseur. Toutefois, on peut réduire l'amplitude d'accommodation $\boldsymbol{A}$ en utilisant un oculaire à réticule.

\section{PHOTOMÉTRIE DES INSTRUMENTS}

Nous allons ici nous intéresser à l'étude des instruments du point de vue énergétique : on va caractériser une source par son intensité ou sa luminance, déterminer le flux d'énergie émis ou reçu, et l'éclairement d'un détecteur. Toutes ces grandeurs vont être définies de façon précise, et reliées entre elles via la géométrie du faisceau. Il sera important de retenir les unités de ces grandeurs.

Quand on parle d'énergie électromagnétique, il est nécessaire de préciser la composition spectrale du rayonnement. Dans un premier temps, nous supposerons les sources monochromatiques : l'unité de flux énergétique sera alors le Watt, et nous parlerons alors de photométrie énergétique. Pour des sources polychromatiques, et des détecteurs associés sensibles dans une certaine plage de longueur d'onde, il est alors nécessaire de sommer les contributions des différentes longueurs d'onde (supposées incohérentes) en pondérant par la composition spectrale de la source et la réponse du détecteur. Dans le cas particulier du domaine visible, en lien avec le détecteur œil, nous verrons brièvement qu'on définit un système d'unités visuelles qui prend en compte la réponse spectrale d'un cil standard : on parle alors de photométrie visuelle. A l'intérieur de ce système d'unités, les grandeurs sont reliées entre elles de la même façon que pour les grandeurs énergétiques. Le lien quantitatif entre les deux systèmes d'unités sera indiqué brièvement.

Remarque : tout ce qui suit est basé sur des raisonnements en intensité, en l'absence de phénomènes d'interférence. 


\subsection{Grandeurs photométriques et géométrie d'un faisceau élémentaire}

\subsection{Flux énergétique $\Phi$}

L'énergie transportée par le rayonnement électromagnétique étant proportionnelle au temps, on définit un flux énergétique $\Phi$ à travers une surface, comme la puissance rayonnée, exprimée en watts.

\subsubsection{Angle solide $\Omega$}

L'angle solide élémentaire $d \Omega$ sous lequel est vue une petite surface $d S$ depuis un point de l'espace $O$ est défini par:

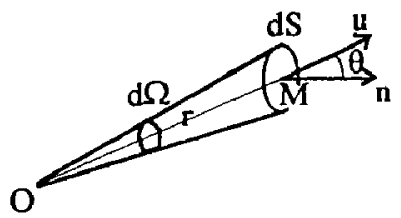

$$
\begin{aligned}
& d \Omega=\frac{\mathbf{u} \cdot \mathbf{n}}{r^{2}} d S \quad \text { avec } r=O M \\
& d \Omega=\frac{d S \cos \theta}{r^{2}}
\end{aligned}
$$

unité d'angle solide : le stéradian (sr)

L'angle solide $\Omega$ sous lequel on voit un objet ou une surface est la somme des angles solides $\mathrm{d} \Omega$ élémentaires. Pour une surface plane circulaire normale à la direction $O C$ (C centre du disque), $\Omega$ est le rapport entre la surface de la calotte sphérique de rayon $R$ et de demiangle au sommet $\alpha$, et le carré de la distance $R$ :

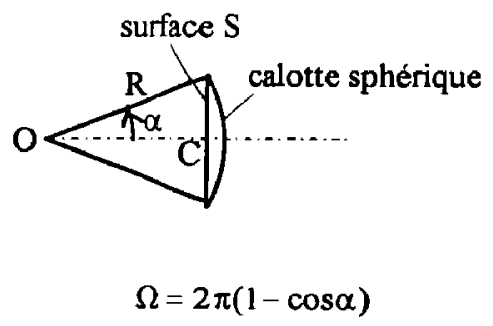

Pour des angles $\alpha$ petits, on a : $\Omega=\pi \alpha^{2}$

L'angle solide cortespondant au demi-espace $\left(\alpha=90^{\circ}\right)$ vaut : $\Omega=2 \pi$

L'angle solide correspondant à tout l'espace $\left(\alpha=180^{\circ}\right)$ vaut : $\Omega=4 \pi$

\subsubsection{Intensité d'une source}

C'est le flux émis par l'ensemble de la source dans une direction donnée, par unité d'angle solide : 


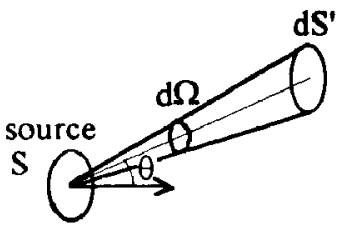

$$
I=\frac{d \Phi}{d \Omega} \quad \text { unité }: \text { watt par stéradian }\left(\mathrm{W} . \mathrm{sr}^{-1}\right)
$$

$I$ dépend en général de la direction d'émission $\theta$. Pour une étoile ponctuelle, $I$ est constant quelle que soit la direction d'émission (source isotrope) tandis que pour un laser I est très grand dans une petite zone angulaire. La variation de $I$ avec la direction d'émission est caractérisée par une indicatrice d'intensité : c'est le lieu des points extrémités d'un vecteur de direction $\theta$ et de norme $I(\theta)$.

Exemples d'allure d'indicatrices d'intensité :

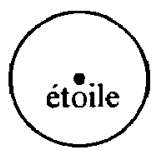

$$
\text { laser }
$$

Pour une source isotrope, la relation entre intensité et flux émis dans un angle solide $\Omega$ s'écrit simplement : $\quad \Phi=I \Omega$

\subsubsection{Luminance d'une source étendue}

Pour une source étendue, on veut pouvoir décrire les variations spatiales de rayonnement de la source. On définit alors la luminance, qui est l'intensité dans une direction donnée, par unité de surface apparente de la source dans cette direction :

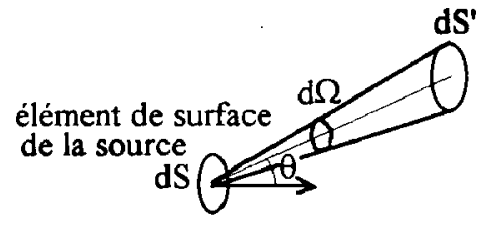

$$
L=\frac{d I}{d S \cos \theta} \quad \text { unité }: \mathrm{W} \cdot \mathbf{s r}^{-1} \cdot \mathrm{m}^{-2}
$$

Le flux élémentaire $d^{2} \Phi$ émis par l'élément de surface de source dS dans un angle solide $d \Omega$ autour d'une direction $\theta$ (infiniment petit d'ordre 2) s'écrit en fonction de la luminance :

$$
d^{2} \Phi=L d \Omega d S \cos \theta
$$

Le fait de définir la luminance par unité de surface apparente de la source permet d'exclure de $L$ la dépendance en $\theta$ qui provient de l'inclinaison de la direction d'observation par rapport à la direction d'émission de la source. Il peut subsister une dépendance de $L$ avec $\theta$, et on la représente alors par une indicatrice de luminance. Pour des sources non uniformes, L va également dépendre du point de la source. 
Toutefois, pour de nombreuses sources, la luminance est en première approximation indépendante de la direction d'émission et uniforme sur toute la surface de la source : de telles sources sont dites lambertiennes. Pour ces sources l'intensité dans la direction $\theta$ s'écrit :

$$
I=\iint_{\text {source }} L d S \cos \theta=L S \cos \theta
$$

Attention une source lambertienne n'est pas une source isotrope ( $I$ dépend de $\theta$ ). Son indicatrice d'intensité a l'allure suivante :

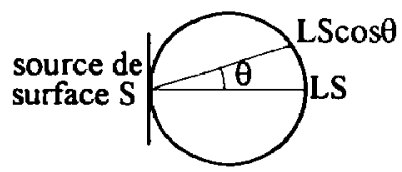

\subsection{5 Étendue géométrique élémentaire}

C'est la caractéristique géométrique du faisceau qui permet de relier la luminance de la source au flux élémentaire $d^{2} \Phi$, émis par un élément $d S$ de la source dans un angle solide $d \Omega$ autour d'une direction $\theta$ :

$$
\begin{gathered}
d^{2} \Phi=L d^{2} G \\
d^{2} G=d \Omega d S \cos \theta
\end{gathered}
$$

unité d'étendue géonétrique : $\mathrm{m}^{2} . \mathrm{sr}$

On peut encore écrire l'étendue géométrique élémentaire sous la forme :
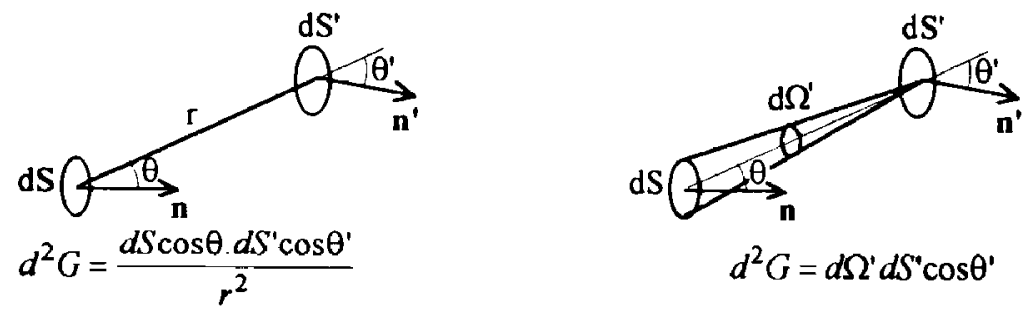

Pour une source lambertienne, le flux émis dans une étendue géométrique G s'écrit tout simplement : $\Phi=L G$

\subsection{6 Éclairement d'un récepteur}

C'est le flux reçu par unité de surface du récepteur :

$$
E=\frac{d \Phi}{d S^{\prime}} \quad \text { unité }: \mathrm{W} \cdot \mathrm{m}^{-2}
$$


où $d \Phi$ est le flux émis par toute la surface de la source dans l'angle solide $d \Omega$ sous lequel on voit l'élément de surface $d S^{\prime}$ du détecteur depuis la source. En utilisant les notations précédentes, on peut écrire :

$$
\begin{aligned}
\mathrm{d} \Phi & =\iint_{\text {source }} \mathrm{d}^{2} \Phi=\mathrm{I}(\theta) \mathrm{d} \Omega=\mathrm{I}(\theta) \frac{\mathrm{d} \mathrm{S}^{\prime} \cos \theta^{\prime}}{\mathrm{r}^{2}} \\
E & =\frac{I(\theta) \cos \theta}{r^{2}} \quad \text { (loi de Bouguer) }
\end{aligned}
$$

L'éclairement diminue comme le carré de la distance à la source et il est maximum lorsque la surface du détecteur est orthogonale à la direction d'émission.

\section{2 Étude photométrique d'un instrument}

\subsection{Flux entrant dans un instrument}

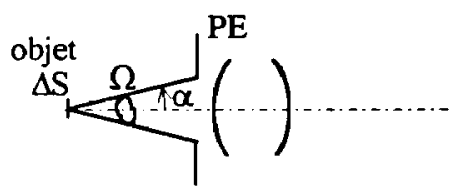

On cherche le flux émis par un petit objet de surface $\Delta S$ centré sur l'axe optique du système et assimilé à une source lambertienne de luminance $L$. On supposera ce petit objet complètement inclus à l'intérieur du champ de pleine lumière de l'instrument. Dans ce cas, le flux entrant est limité par la pupille d'entrée du système, caractérisée par l'angle d'ouverture $\alpha$. On peut done écrire ce flux sous la forme :

$$
\Phi_{\text {entrant }}=L G
$$

où $G$ est l'étendue géométrique du faisceau limité par la surface source d'un côté et la surface de la pupille d'entrée de l'autre. $G$ s'écrit donc:

$$
\mathrm{G}=\iint_{\mathrm{PE}} \Delta \mathrm{S} \cos \theta \mathrm{d} \Omega
$$

Pour calculer $G$, on découpe la pupille d'entrée en couronnes comprises entre les rayons $\mathrm{R}$ et $R+d R$, correspondant à des angles d'ouverture compris entre $\theta$ et $\theta+d \theta$ :

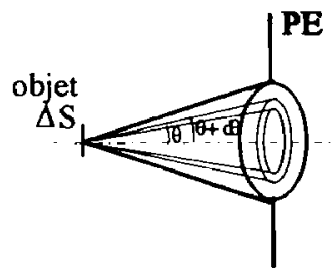


L'angle solide $d \Omega$ correspondant à une telle couronne vaut : $d \Omega=2 \pi \sin \theta d \theta$, d'où

$$
\begin{gathered}
\mathbf{G}=\int_{0}^{\Phi_{0}} \Delta \mathrm{S} \cos \theta \cdot 2 \pi \sin \theta \mathrm{d} \theta=\pi \Delta \mathrm{S}^{2} \sin ^{2} \alpha \\
\Phi_{\text {entrent }}=\pi L \Delta S \sin ^{2} \alpha
\end{gathered}
$$

\subsubsection{Flux sortant de l'instrument. Transmission.}

L'objet étant à l'intérieur du champ de pleine lumière de l'instrument, tous les rayons issus de cet objet et entrant dans la pupille d'entrée vont ressortir du système. Le flux sortant va donc être égal au flux entrant, à la transmission près des optiques ( $\tau$ transmission en énergie) :

$$
\Phi_{\text {sortant }}=\tau \Phi_{\text {entrant }}
$$

On peut également écrire le flux sortant de façon analogue au flux entrant, en fonction de la luminance $L$ ', de la surface $\Delta S$ ' de l'image et de l'angle d'ouverture $\alpha$ ' dans l'espace image :

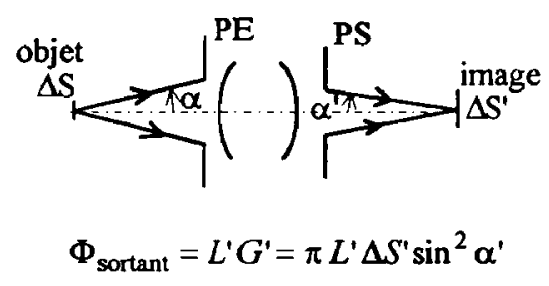

\subsubsection{Conservation de l'étendue optique à travers un instrument}

Considérons des surfaces objet et image circulaires de rayon y et $y^{\prime}$ respectivement : $\Delta S=\pi y^{2}, \Delta S^{\prime}=\pi y^{\prime 2}$. Si le système optique est stigmatique et aplanétique (donc en particulier dans le domaine de l'approximation de Gauss), on a la relation des sinus d'Abbe :

$$
\begin{aligned}
& n y \sin \alpha=n^{\prime} y^{\prime} \sin \alpha^{\prime} \\
& \text { ou encore : } \quad n^{2} \Delta S \sin ^{2} \alpha=n^{\prime 2} \Delta S^{\prime} \sin ^{2} \alpha^{\prime} \\
& n^{2} G=n^{\prime 2} G^{\prime}
\end{aligned}
$$

L'étendue optique $n^{2} G$ est conservee à la traversée d'un système optique aplanétique (l'étendue géométrique également dans le cas courant où $n=n$ ).

En d'autres termes, on ne peut pas à la fois réduire la dimension transverse d'un faisceau et sa divergence (on peut par contre les augmenter toutes deux si le système n'est pas aplanétique). 


\subsubsection{Conservation de la luminance}

D'après la relation entre flux entrant et flux sortant on a : $\Phi_{\text {sortant }}=L^{\prime} G^{\prime}=\tau \Phi_{\text {entrant }}=\tau L G$ En utilisant la conservation de l'étendue optique, on obtient alors :

$$
L^{\prime}=\tau \frac{n^{\prime 2}}{n^{2}} L
$$

Il y a donc conservation de la luminance entre objet et image dans le cas où les milieux extrêmes sont identiques $(n=n)$ et où le système optique est sans pertes $(\tau=1)$. Rappelons également que l'objet doit se trouver à l'intérieur du champ de pleine lumière de l'instrument pour que la relation ci-dessus soit vérifiée.

\subsection{5 Éclairement dans le plan de l'image, sur l'axe.}

Si on place un écran ou un détecteur dans le plan de l'image, une petite surface dS' de ce plan, centrée sur l'axe, va recevoir le flux sortant du système :

$$
\begin{gathered}
d \Phi_{\text {sortant }}=\pi L^{\prime} d S^{\prime} \sin ^{2} \alpha^{\prime} \\
E=\frac{d \Phi_{\text {sortant }}}{d S^{\prime}}=\tau \frac{n^{\prime 2}}{n^{2}} \pi L \sin ^{2} \alpha^{\prime}
\end{gathered}
$$

Dans le cas courant où $n=n^{\prime}$, l'éclairement s'exprime :

$$
E=\tau \pi L \sin ^{2} \alpha^{\prime}
$$

On voit notamment que l'éclairement dans le plan image ne dépend que de l'angle d'ouverture $\alpha^{\prime}$, et pas de la taille ni de la position de l'image.

\subsubsection{Cas d'un appareil photo réglé sur l'infini}

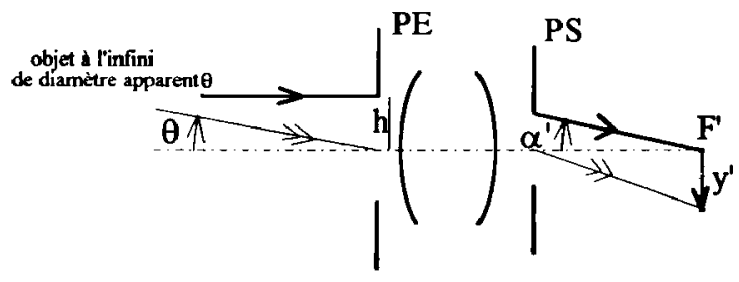

Si l'appareil est aplanétique avec $n=n^{\prime}=1$, on a la relation des sinus d'Abbe dans le cas d'un objet à l'infini :

ou encore : $\quad-\frac{h}{\sin \alpha^{\prime}}=\frac{y^{\prime}}{\theta}=$ cste (indépendante de $\theta$ ) 
Cette relation étant vérifiée quel que soit le couple $\left(\theta, y^{\prime}\right)$, elle l'est en particulier dans le cas des petits angles $\theta$ (approximation de Gauss). Dans ce cas, on a $y^{\prime}=f^{\prime} \theta$, où $f^{\prime}$ est la focale image de l'appareil photo. On a donc :

$$
\varnothing_{P E}=2 f^{\prime}\left|\sin \alpha^{\prime}\right|
$$

ou encore en terme de nombre d'ouverture $\mathbf{N}$ de l'appareil photo :

$$
N=\frac{1}{2\left|\sin \alpha^{\prime}\right|}
$$

Notons que, lorsque l'appareil photo est réglé sur une distance finie, celle-ci est en général très grande devant la focale du système (typiquement $f^{\prime}=50 \mathrm{~mm}$ et on vise au minimum à 1 $m$ ), de sorte que la relation ci-dessus entre le nombre d'ouverture et l'angle $\alpha$ ' reste approximativement vraie (sinc' est très légèrement inférieur à $1 / 2 \mathrm{~N}$ ).

Finalement l'éclairement dans le plan de l'image sur l'axe s'écrit pour l'appareil photo :

$$
E=\frac{\pi \tau L}{4 N^{2}}
$$

7.2.5.2 Cas de l'observation à l'ceil : éclairement rétinien. Modélisons l'œil par une lentille mince séparant deux milieux d'indice $n$ (l'air en général) et $n^{\prime}$ (humeur aqueuse). On sait que lorsque l'œil accommode, la taille de l'image y' ne dépend que de l'angle sous lequel on voit l'objet, c'est-à-dire que la lentille change de focale mais la distance lentille-rétine reste la même (environ $20 \mathrm{~mm}$ ) :

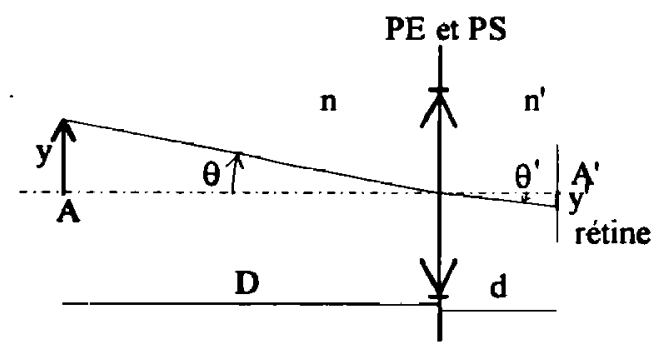

$$
y^{\prime}=\theta^{\prime} d=\theta \frac{n}{n^{\prime}} d \text { ne dépend que de } \theta \text { si } d=\text { cste }
$$

La pupille étant sur la lentille, l'angle d'ouverture image vaut : $\alpha^{\prime}=\emptyset_{\text {owi }} / 2 d$, où $\emptyset_{\text {cil }}$ est le diamètre de la pupille de l'œil ( $\alpha$ ' de l'ordre de $0.05 \mathrm{rad}$ en vision de jour). On obtient donc l'expression de l'éclairement de la rétine en fonction de la luminance de l'objet $\mathrm{L}$ et de la transmission de l'œil $\tau_{\text {ceil }}$ : 


$$
E_{\text {re tinc }}=\pi \tau_{\text {ocil }} \frac{n^{\prime 2}}{n^{2}} L \frac{\varnothing_{\text {oal }}^{2}}{4 d^{2}}
$$

Finalement l'éclairement rétinien est proportionnel à la luminance de l'objet et à la surface éclairée de la pupille de l'œil (qui peut être une fraction de la pupille si l'œil est placé derrière un instrument dont la pupille de sortie est plus petite que la pupille d'œil).

\subsubsection{Variation d'éclairement dans le champ d'un instrument}

7.2.6.1 A l'intérieur du champ de pleine lumière. Considérons un élément de surface objet $\Delta \mathbf{S}$ centré autour d'un point $\mathbf{B}$ hors d'axe, repéré par l'angle $\theta$ depuis le centre de la pupille d'entrée. Son image est une petite surface $\Delta S^{\prime}$ centrée autour de l'image $B$ ' de $B$, repérée par l'angle $\theta$ ' depuis le centre de la pupille de sortie.

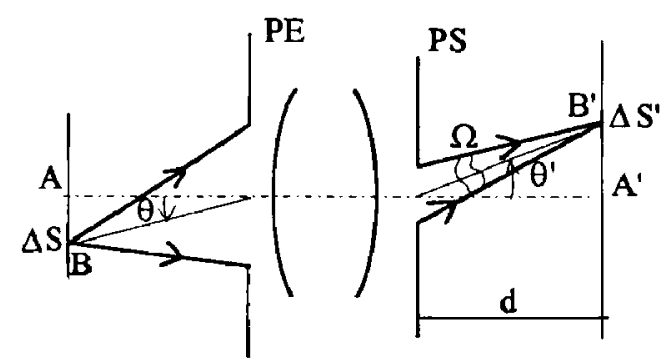

Tant qu'on est à l'intérieur du champ de pleine lumière, tous les rayons s'appuyant sur la pupille d'entrée sont toujours transmis à travers le système (pas d'obturation par une lucarne). La pupille de sortie du système est donc uniformément éclairée et peut être considérée comme une source secondaire de luminance uniforme $L_{P S}$, qui vient éclairer le plan image. Si l'on suppose que l'angle solide sous lequel est vue cette pupille de sortie depuis un point du plan image est petit, on pourra écrire le flux requ par $\Delta S^{\prime}$ sous la forme :

$$
\Phi\left(\mathrm{B}^{\prime}\right)=L_{\mathrm{PS}} \frac{S_{\mathrm{PS}} \cos \theta^{\prime} \cdot \Delta S^{\prime} \cos \theta^{\prime}}{\left(d / \cos \theta^{\prime}\right)^{2}}
$$

Dans les mêmes conditions, le flux reçu par une surface $\Delta S^{\prime}$ autour du point $\mathrm{A}^{\prime}$ sur l'axe s'écrit :

$$
\Phi\left(\mathrm{A}^{\prime}\right)=L_{\mathrm{PS}} \frac{S_{\mathrm{PS}} \cdot \Delta S^{\prime \prime}}{d^{2}}
$$

Pour obtenir les éclairements, il suffit de diviser les flux par la surface réceptrice $\Delta S^{\prime}$, et on obtient la relation entre éclairement sur l'axe $E\left(A^{\prime}\right)=E_{0}$ et éclairement hors d'axe

$$
E\left(\theta^{\prime}\right)=E_{0} \cos ^{4} \theta^{\prime}
$$


Notons que cette diminution d'éclairement est très lente (notamment par rapport à l'effet de vignettage hors du champ de pleine lumière) et que dans la pratique l'effet de la variation des transmissions des optiques avec l'angle d'incidence peut s'y ajouter.

7.2.6.2 Au-delà du champ de pleine lumière. Lorsqu'on s'écarte dans le champ au-delà de la limite du champ de pleine lumière, la pupille de sortie n'est plus uniformément éclairée. Une partie du faisceau est obturé par la (ou les) lucarne(s). Cet effet, encore appelé vignetage, vient s'ajouter à l'effet géométrique précédent et il a pour effet de diminuer beaucoup plus rapidement l'éclairement dans le plan de l'image. Lorsqu'on atteint le bord du champ total, plus aucun rayon ne traverse le système et l'éclairement s'annule.

Pour se faire une idée de l'allure de l'éclairement entre le bord du champ de pleine lumière et le bord du champ total, considérons le cas d'un système à deux diaphragmes, et plaçons-nous dans l'espace image.

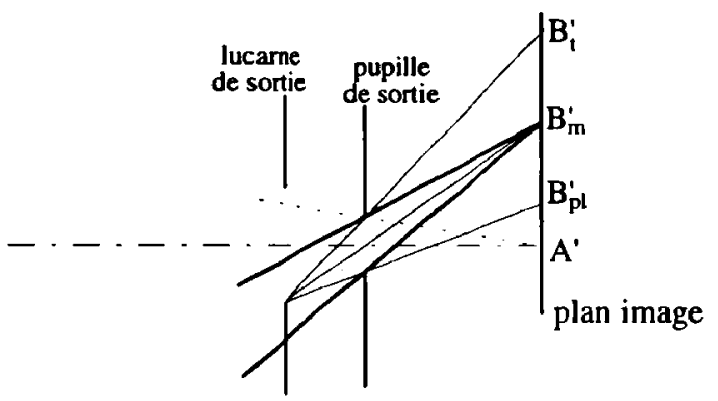

Remarquons tout d'abord que dans cette situation à 2 diaphragmes, le champ moyen $B^{\prime}{ }_{m}$ est au milieu des limites de champ de pleine lumière $B^{\prime}{ }_{p l}$ et de champ total $B^{\prime}{ }_{t}$. Cherchons alors l'éclairement autour du point $B^{\prime}{ }_{m}$. En l'absence de lucarne, on aurait le cône de rayons s'appuyant sur le contour de la pupille de sortie (représenté en traits épais sur la figure) et l'éclairement vaudrait $E_{0} \cos ^{4} \theta_{m}^{\prime}$. La lucarne vient alors obturer une fraction du flux correspondant au rapport des surfaces par exemple dans le plan de la lucarne :

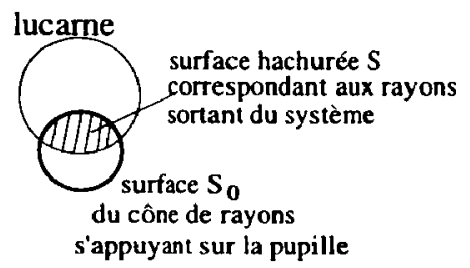

On voit qu'au niveau du champ moyen le rapport $S / S_{0}$ est inférieur à 0.5 . Finalement on peut représenter l'allure de l'éclairement en fonction de l'angle de champ $\theta$ ' : 


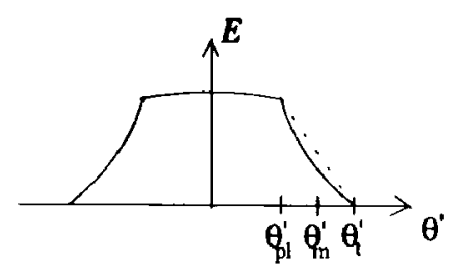

\subsubsection{Unités de photométrie visuelle}

Comme il a été dit dans l'introduction de cette partie, les unités fondamentales de photométrie sont les unités énergétiques dont l'unité de base est le watt. Le système d'unités visuelles en dérive par le biais d'une sommation sur les longueurs d'onde visibles, pondérée par la réponse spectrale de l'œil standard (différente suivant le jour et la nuit). Par exemple, pour une lumière monochromatique de longueur d'onde $550 \mathrm{~nm}$ (maximum de sensibilité de l'œil en vision de jour), on a 1 wait pour 683 lumens.

Le table:au ci-dessous donne les unités visuelles et leurs correspondants dans le système énergétique :

\begin{tabular}{|c|c|c|}
\hline GRANDEURS & unité énergétique & unité visuelle \\
\hline Flux & watt & lumen $\mathrm{Im}$ \\
\hline Intensité & W.sr ${ }^{-1}$ & candela $\mathrm{cd}=\operatorname{lm} . \mathrm{sr}^{-1}$ \\
\hline Luminance & $\mathrm{W} \cdot \mathrm{m}^{-2} \cdot \mathrm{sr}^{-1}$ & nit $=$ cd.m. $m^{-2}$ \\
\hline Eclairement & W. $\mathrm{m}^{-2}$ & $\operatorname{lux}=\operatorname{lm} \cdot \mathrm{m}^{-2}$ \\
\hline
\end{tabular}

Notons que les relations entre les différentes grandeurs restent valables à l'intérieur de chaque système d'unités. On aura donc intérêt à rester dans le système d'unités que l'on a fixé au départ.

\subsection{Clarté et luminosité des instruments}

La clarté est une propriété de l'instrument qui caractérise son rendement photométrique. Sa définition dépend du type d'instrument, visuel ou objectif, mais aussi du type d'image réalisé, étendue ou ponctuelle.

\subsubsection{Clarté des instruments visuels}

La quantité qui va compter dans ce cas est le rapport des flux reçus par chaque cellule rétinienne, avec ou sans l'instrument. On distingue alor's deux cas :

7.3.1.1 Les images à l'œeil nu et par l'instrument couvrent plusieurs cellules rétiniennes (objet étendu). Dans cette situation le flux reçu par chaque cellule rétinienne est directement proportionnel à l'éclairement, et la clarté est donc le rapport des éclairements :

$$
C=\frac{E^{\prime}}{E}=\frac{\text { éclairement de l'image rétinienne avec l'instrument }}{\text { éclairement de l'image rétinienne à l 'ceil nu }}
$$


- Si c'est la pupille d'ail qui fixe l'ouverture dans les deux cas (c'est-à-dire si le diamètre de la pupille de sortie de l'instrument $\varnothing_{\text {PS }}$ est supérieur à celui de la pupille d'œil $\varnothing_{\text {ail }}$ ), on a :

$$
C=\frac{\pi L^{\prime} \varnothing_{\text {oeil }}^{2}}{\pi L \varnothing_{\text {oeil }}^{2}}=\frac{L^{\prime}}{L}=\tau \quad \text { la clarté est égale à la transmission de l'instrument }
$$

C'est par exemple le cas d'une loupe où c'est forcément l'œil qui limite l'ouverture.

- Si c'est l'instrument qui fixe l'ouverture $\left(\varnothing_{\mathrm{PS}}<\varnothing_{\text {cail }}\right)$, on a :

$$
C=\tau\left(\frac{\varnothing_{\text {PS }}}{\varnothing_{\text {oeil }}}\right)^{2}<\tau
$$

On voit que du point de vue photométrique on a intérêt à prendre la pupille de sortie de l'instrument voisine de celle de l'œil et à augmenter au maximum par des traitements antireflets la valeur de la transmission $\tau$. Dans le meilleur des cas, la clarté sera égale à 1 , c'est-à-dire que la luminosité de l'objet sera la même avec ou sans instrument (par contre d'autres qualités auront été modifiées).

Dans le cas d'un microscope, on peut, grâce à la relation d'aplanétisme appliquée au microscope complet, relier le diamètre de la pupille de sortie à l'ouverture numérique $n \sin \alpha$ et la puissance $\boldsymbol{P}$ sous la forme :

$$
\varnothing_{\mathrm{ps}}=2 \frac{n \sin \alpha}{P}
$$

Il apparaît alors une puissance caractéristique $P_{c}$, dite puissance équipupillaire : $P_{e}=2 n \sin \alpha / \varnothing_{\text {oeil }}(500 n \sin \alpha$ dioptries pour une pupille d'œil de diamètre $4 \mathrm{~mm}$ ), telle que :

pour $\mathrm{P}<\mathrm{P}_{e}$ la clarté est constante et maximale égale à $\tau$

$$
\text { pour } \mathrm{P}>\mathrm{P}_{\mathrm{e}} \text { la clarté chute quadratiquement }: \mathrm{C}=\tau\left(\frac{\mathrm{P}_{\mathrm{e}}}{\mathrm{P}}\right)^{2}
$$

Par un raisonnement analogue dans le cas d'une lunette astronomique de grossissement $G$ observant des objets étendus (planètes par exemple), on a :

$$
G=\frac{\varnothing_{\mathrm{PE}}}{\varnothing_{\mathrm{PS}}} \quad \text { grossissement équipupillaire } G_{e}=\frac{\varnothing_{\mathrm{PE}}}{\varnothing_{\text {oeil }}}
$$

Pour $\mathrm{G}<\mathrm{G}_{\mathrm{e}}$ on a $\mathrm{C}=\tau$, puis pour $\mathrm{G}>\mathrm{G}_{e}, \mathrm{C}=\tau\left(\mathrm{G}_{\mathrm{e}} / \mathrm{G}\right)^{2}$ : pour une lunette terrestre ou des jumelles, où on veut la clarté la plus grande possible on se limite à des grossissements inférieurs au grossissement équipupillaire $\left(\varnothing_{\mathrm{PS}}>\varnothing_{\text {ocil }}\right)$.

7.3.1.2 Les images à l'œil nu et à travers l'instrument sont ponctuelles (plus petites qu'une cellule rétinienne). On doit alors revenir aux flux et la clarté s'exprime : 


$$
\begin{aligned}
& C=\frac{\Phi^{\prime}}{\Phi}=\frac{\text { flux recu par la réline avec l'instrument }}{\text { flux recu par la rétine à l 'œil nu }} \\
& C=\frac{E^{\prime} \cdot S^{\prime}}{E \cdot S}=\frac{E^{\prime}}{E} \frac{S^{\prime}}{S}=\frac{E^{\prime}}{E}\left(\frac{y^{\prime}}{y}\right)^{2}=\frac{E^{\prime}}{E}\left(\frac{q^{\prime}}{q}\right)^{2}=\frac{E^{\prime}}{E} G^{2}
\end{aligned}
$$

où $S$ et $S^{\prime}$ sont les surfaces de l'image sur la rétine à l'œil nu et avec l'instrument respectivement, $\theta$ et $\theta$ ' sont les angles sous lesquels l'œil voit l'image sans ou avec instrument et $G$ est le grossissement de l'instrument.

On obtient donc dans le cas d'objets et d'images ponctuels une clarté $G^{2}$ fois supérieure à la clarté obtenue dans le cas d'un objet étendu : elle peut donc être très supérieure à 1 . On met ainsi en évidence l'intérêt d'observer des étoiles avec une lunette de fort grossissement : même si l'image reste ponctuelle, on gagne en luminosité ce qui permet de détecter des étoiles plus faibles.

$\mathrm{Si}$ on reprend le cas du microscope ou de la lunette observant cette fois des objets ponctuels (ultramicroscopie ou observation d'étoiles ponctuelles), on voit que la clarté va maintenant croître en $G^{2}$ jusqu'à la valeur équipupillaire, puis être constante égale à $\tau G_{c}{ }^{2}$ audelà. On voit donc que pour optimiser la clarté pour les deux types d'observations, on a intérêt à choisir une valeur de grossissement (ou de puissance) proche de la valeur équipupillaire.

\subsubsection{Luminosité des instruments de projection}

L'objet est dans ce cas caractérisé par sa luminance $\mathrm{L}$ et l'image par son éclairement. On parle de luminosité plutôt que de clarié pour l'instrument :

$$
C=\frac{E^{\prime}}{L}=\frac{\text { eclairement de l'iniage }}{\text { luminance de l'obiet }}=\pi \tau \sin ^{2} \alpha^{\prime}
$$

Elle ne dépend que du facteur de transmission et de l'ouverture dans l'espace image.

\section{Bibliographie}

Il existe bien sûr de nombreux ouvrages traitant de l'optique géométrique et instrumentale. Je n'en citerai ici que quelques-uns qui m'ont été utiles pour préparer ce cours :

- Maréchal A., Imagerie géométrique (éditions de la Revue d'Optique théorique et instrumentale, Paris, 1952), Traité d'Optique Instrumentale, Première section, Tome I.

- Moussa A. et Ponsonnet P., Cours de Physique I, Optique (éditions Desvigne, Paris, 1992).

- Perez J. Ph., Optique géométrique, ondulatoire et polarisation (Masson, Paris, $4^{e}$ edition 1994)

- Hecht E., Optics (Addison-Wesley, USA, $2^{\text {nd }}$ ed 1990).

- Feynman, Leighton et Sands, Le cours de Physique de Feynman (Interéditions, Paris, 1979), chapitres 26 à 33 du tome Mécanique 2. 